\title{
GENERATORS FOR A COMPLEX HYPERBOLIC BRAID GROUP
}

\author{
DANIEL ALLCOCK AND TATHAGATA BASAK
}

\begin{abstract}
We give generators for a certain complex hyperbolic braid group. That is, we remove a hyperplane arrangement from complex hyperbolic 13space, take the quotient of the remaining space by a discrete group, and find generators for the orbifold fundamental group of the quotient. These generators have the most natural form: loops corresponding to the hyperplanes which come nearest the basepoint. Our results support the conjecture that motivated this study, the "monstrous proposal", which posits a relationship between this braid group and the monster finite simple group.
\end{abstract}

\section{Contents}

1. Introduction

2. Background, conventions, notation

3. Meridians and homotopies between them

4. Finitely many generators based "at" a cusp

5. Twenty-six generators based "at" a cusp

6. Change of basepoint

Appendix A. How eight totally real triangles meet the mirrors

Appendix B. How two complex triangles meet the mirrors 43

References

\section{INTRODUCTION}

We are interested in finding generators and relations for complex hyperbolic braid groups. Complex hyperbolic space $\mathbb{B}^{n}$ is a complex manifold, diffeomorphic to the unit ball in $\mathbb{C}^{n}$, and closely analogous to real hyperbolic space. Our braid groups, which we called braid-like in $\mathrm{AB}$, arise by removing a locally finite arrangement of complex hyperplanes, quotienting the remaining space by the action of a discrete group, and then taking the fundamental group. This is closely analogous to a wellknown construction of the classical braid group [FN]: remove the hyperplanes $x_{i}=$ $x_{j}$ from $\mathbb{C}^{n}$, and then quotient by the symmetric group $S_{n}$. A similar construction, with $S_{n}$ replaced by any other Coxeter group, yields the Artin groups $\left.\mathrm{Br} 1\right] \mathrm{vdL}$. In a different direction, replacing $S_{n}$ by a finite complex reflection group leads to the complex braid groups, whose presentations and key properties were worked out

Date: September 19, 2016.

2010 Mathematics Subject Classification. Primary: 57M05; Secondary: 20F36, 52C35, 32S22.

First author supported by NSF grant DMS-1101566 and a Simons Foundation Collaboration Grant. 
only recently $[\mathrm{Be}$. In a sense we are generalizing the classical braid group in both these ways simultaneously.

Our main result, theorem 1.2 gives a natural set of generators for a particular complex hyperbolic braid group coming from $\mathbb{B}^{13}$. Finding enough relations that give a presentation of the fundamental group appears to be rather harder. We will explain why this example is interesting, but to set the scene we begin with our broader motivations. In a sense these are detours, since we do not develop them further in this paper.

The first motivation involves singularity theory. The Artin groups of types $A_{n}$, $D_{n}$ and $E_{n}$ appear in nature as the fundamental groups of the discriminant complements of the simple singularities, which are also called $A_{n}, D_{n}$ and $E_{n}$. Under a technical assumption that often holds, this has the following consequence. Suppose given a family of complex varieties over a base variety $B$, write $B_{0} \subseteq B$ for the set of smooth fibers, and suppose the fiber over $b \in B$ has some simple singularities but no worse singularities. Then these singularities have types $A_{n}, D_{n}$ and $E_{n}$ for some choices of subscripts, and there is a neighborhood $U$ of $b$ such that $\pi_{1}\left(B_{0} \cap U\right)$ is the direct product of the corresponding Artin groups. Informally: $A_{n}, D_{n}$ and $E_{n}$ Artin groups appear "locally" in the fundamental groups of "most" families of algebraic varieties. For example, by considering families of Riemann surfaces, one automatically expects suitable elements of mapping class groups to satisfy the braid relation - an expectation fulfilled by Dehn twists around curves that meet just once (and transversely). See [Li], L3], (3.5)-(3.7) of [ACT1, and lemma 1.5 and theorem 7.1 of $\mathrm{ACT} 2$ for examples and applications of these ideas; the needed technical assumption is that the family $B$ should provide a simultaneous versal deformation of all the singularities.

Now, singularities well beyond the simple ones have been classified, and the next-least complicated ones are the "affine" singularities $\tilde{A}_{n}, \tilde{D}_{n}, \tilde{E}_{n}$. From the notation one naturally expects that the fundamental groups of their discriminant complements are the corresponding affine Artin groups. But this is not so vdL. And more-singular singularities have discriminant complements whose fundamental groups are even further from the Artin groups. For example, [Lö, Thm. 4.3] gives presentations in the case of the Brieskorn-Pham singularities $x_{1}^{d_{1}}+\cdots+x_{n}^{d_{n}}=0$, where each $d_{i}$ is at least 2 . These presentations include Artin relations but also additional relations. But in many cases, for example [L1] [L2, §10] [La], these fundamental groups are braid groups in our sense, with $\mathbb{B}^{n}$ replaced by the symmetric space $\mathrm{SO}(2, n) / S(\varnothing(2) \times \varnothing(n))$. The reasoning we used for simple singularities generalizes to any singularity, so these new braid groups appear automatically when considering families of complex varieties. In particular, they appear in nature in a way that the infinite-type Artin groups do not. Brieskorn $\mathrm{Br} 2$ has asked for presentations for more discriminant complements, and we hope our methods will contribute to this. We regard braid groups coming from hyperplane arrangements in $\mathbb{B}^{n}$ as an easier analogue of the $\mathrm{SO}(2, n) / S(\varnothing(2) \times \varnothing(n))$ case, hence a test bed for our ideas.

Our second motivation is the braid groups of the finite complex reflection groups. The known presentations $\mathrm{Be}$ are obtained from Lefschetz pencils, rather than directly from the arrangement of the hyperplanes. In the case of Artin groups, the standard basepoint lies in the interior of the Weyl chamber, and the standard generators are the following paths, called "meridians". Each starts at the basepoint, 
moves directly toward a facet $F$ of the chamber until close to it, then travels along a semicircle around the complexification of $F$, and then moves directly to the image of the basepoint under the reflection across $F$. Although this is not a loop, it becomes one after quotienting by the Weyl group. In this way the standard generators correspond to the mirrors nearest the basepoint. We hope that there are analogous good generating sets for the complex braid groups. The Weyl chamber is not available in this context, but there may still be natural basepoints (possibly the "Weyl vectors" of [Ba2]), and generators like those for Artin groups, coming from the mirrors nearest the basepoint.

Now we discuss the braid group that is the subject of this paper. It comes from a group $P \Gamma$ acting on $\mathbb{B}^{13}$ with finite covolume, generated by triflections (complex reflections of order 3 ). The hyperplane arrangement $\mathcal{H}$ is the union of the mirrors of the triflections. In a sense this is the hardest example available, because $n=13$ is the highest dimension for which there is a known finite-covolume complex hyperbolic reflection group on $\mathbb{B}^{n}$. Our main result, theorem 1.2, concerns a particular basepoint $\tau \in \mathbb{B}^{13}$ and the meridians based there. Meridians are defined below, and more generally in section 3, but they are similar to the Artin group case. The only difference is that the circular-arc portion of the path is only one third of a circle, not half, because the complex reflections have order 3 , not 2. Our main result is that the braid group $G_{\tau}=\pi_{1}^{\text {orb }}\left(\left(\mathbb{B}^{13}-\mathcal{H}\right) / P \Gamma, \tau\right)$ is generated by the meridians associated to the 26 mirrors nearest $\tau$. The notation $\pi_{1}^{\text {orb }}$ indicates the orbifold fundamental group, which is needed because $P \Gamma$ does not act freely on $\mathbb{B}^{13}-\mathcal{H}$. See section 2.9 for our conventions about orbifold fundamental groups.

The motivation to study this particular example is the first author's "monstrous proposal", namely the following conjecture concerning the sporadic finite simple group $M$ known as the monster. See [A2] for background.

Conjecture 1.1 (Monstrous Proposal [A2]). The quotient of $G_{\tau}$, by the subgroup $N$ normally generated by the squares of the meridians, is isomorphic the "bimonster" $B=(M \times M) \rtimes \mathbb{Z} / 2$, where $M$ is the monster finite simple group and $\mathbb{Z} / 2$ acts by exchanging the factors in the obvious way.

A known presentation of $B$ has 26 generators of order 2, corresponding to the points and lines of $P^{2} \mathbb{F}_{3}$. Two of these generators braid or commute according to whether the corresponding points/lines are incident in the usual sense of projective geometry. There is one additional relation; see CSi. The amazing coincidence is that $G_{\tau}$ 's 26 generators may be indexed by the points and lines of $P^{2} \mathbb{F}_{3}$ in the same way, and they satisfy exactly the same commutation and braid relations [Ba3, Thm. 4.7]. And although it has not yet been verified, there are good geometric grounds to expect that $G_{\tau}$ 's generators also satisfy the additional bimonster relation, or one equivalent to it modulo the (meridian) $)^{2}=1$ relations. Assuming this, $G_{\tau} / N$ is a quotient of $B$, hence is isomorphic to $B$ or $\mathbb{Z} / 2$ or the trivial group. To prove conjecture 1.1 one would need to rule out the cases $G_{\tau}=\mathbb{Z} / 2$ or the trivial group. So we regard theorem 1.2 as significant progress toward conjecture 1.1.

Now we develop just enough background to make precise the objects we have discussed; for additional background see section 2. We write $\mathcal{E}$ for the ring $\mathbb{Z}\left[e^{2 \pi i / 3}\right]$ of Eisenstein integers. The central character in the paper is a particular hermitian $\mathcal{E}$-lattice $L$, namely the unique one of signature $(13,1)$ which equals $\sqrt{-3}$ times its dual lattice. A concrete model for $L$ is the $\mathcal{E}$-span of the 13 "point-roots" like 
$(0 ; \sqrt{-3}, 0, \ldots, 0)$ and the 13 "line-roots" like $(1 ; 1,1,1,1,0, \ldots, 0)$. Here we are using the standard hermitian form of signature $-+\cdots+$ on $\mathbb{C}^{14}$, with the last 13 coordinates indexed by the points of $P^{2} \mathbb{F}_{3}$, and each line-root having coordinates 1 at the four points of a line of $P^{2} \mathbb{F}_{3}$. The complex ball $\mathbb{B}^{13}$ is the set of complex lines of negative norm in $L \otimes_{\mathcal{E}} \mathbb{C}$. A root means a lattice vector of norm 3 , for example a point- or line-root. If $r$ is a root, then we write $R_{r}$ for the triflection in $r$, meaning the isometry of $L$ which multiplies $r$ by $e^{2 \pi i / 3}$ and fixes $r^{\perp}$ pointwise. The mirror of this complex reflection means the fixed point set in $\mathbb{B}^{13}$. The hyperplane arrangement $\mathcal{H}$ is the union of the mirrors, and $P \Gamma$ is the subgroup of Aut $\mathbb{B}^{13}$ generated by the triflections (which is the full projective isometry group of $L$ ).

The mirrors of the 13 point-roots meet orthogonally at a point of $\mathbb{B}^{13}$, and similarly for the 13 line-roots. The basepoint $\tau$ is the midpoint of the segment joining these two points, and the mirrors nearest $\tau$ are exactly these 26 mirrors. For any point- or line-root $r$, the corresponding meridian in $G_{\tau}=\pi_{1}^{\text {orb }}\left(\left(\mathbb{B}^{13}-\mathcal{H}\right) / P \Gamma, \tau\right)$ is represented by the following three-part path. Let $p$ be the projection of $\tau$ to the mirror $r^{\perp}$, and let $U$ be an open ball centered at $p$, small enough so that the only mirror it meets is $r^{\perp}$. Let $d$ be a point of the geodesic $\overline{\tau p}$, lying in $U$ and different from $p$. The first part of the meridian is the geodesic $\overline{\tau d}$. The second part is the circular arc from $d$ to $R_{r}(d)$, centered at $p$ and positively oriented in the complex geodesic containing $\overline{\tau p}$. The third part of the meridian is the geodesic $\overline{R_{r}(d) R_{r}(\tau)}$. We call these elements of $G_{\tau}$ the point- and line-meridians. Now our main theorem has precise meaning:

Theorem 1.2 (Main theorem). The 13 point-meridians and 13 line-meridians generate the orbifold fundamental group of $\left(\mathbb{B}^{13}-\mathcal{H}\right) / P \Gamma$, based at $\tau$.

We announced this in $\mathrm{AB}$, and our starting point for the proof is theorem 1.5 of that paper. That result gives a specific infinite generating set for $G_{\rho}$, which is this same orbifold fundamental group, but based at a cusp $\rho \in \partial \mathbb{B}^{13}$ of $P \Gamma$. This generating set consists of the meridians based at $\rho$ and corresponding to the (infinitely many) mirrors which come closest to $\rho$. (See section 3 for what we mean by taking the basepoint at a cusp, by the meridians based there, and what it means for a mirror to come closest to $\rho$. There are no surprises, but some care is needed.)

Given this starting point, our first step is to exhibit a finite subset of these meridians, which is a generating set. This occupies section 4, and the key argument concerns generators for the $P \Gamma$-stabilizer of $\rho$. Our second step is show that the meridians corresponding to the point- and line-roots, but based at $\rho$ rather than $\tau$, are also a generating set. This is section 5, and the method is to show that the subgroup of $G_{\rho}$ they generate contains all the generators from section 4 . Finally, in section 6] we show that moving the basepoint from $\rho$ to $\tau$, along the geodesic $\overline{\rho \tau}$, identifies the point- and line-meridians based at $\rho$ with those based at $\tau$ in the obvious way. This implies theorem 1.2. At heart, all of our arguments involve concrete homotopies between various paths in $\mathbb{B}^{13}-\mathcal{H}$. Besides setting up our general definition of meridians, section 3 contains several theorems saying that such homotopies exist, provided that certain totally geodesic triangles in $\mathbb{B}^{13}$ miss $\mathcal{H}$. The paper rests on the verification of this property for a total of 10 triangles, in the appendices.

Part of this verification relies on computer calculation; we also used the computer to verify the paper's many hand-calculations involving vectors in $\mathbb{C}^{14}$. These calculations are involved enough that a reader skimming over them might imagine 
that the main theorem is a numerical accident. In fact, behind most of these calculations lurk special properties of the Leech lattice, such as inequalities that are exactly what is needed to complete a proof. So we will emphasize these properties when they arise; they lend the calculations a certain sense of inevitability.

The authors are grateful to the RIMS (Kyoto U.) for its hospitality during part of this work.

\section{BACKGround, CONVEntions, Notation}

2.1. Eisenstein lattices. Let $\omega=e^{2 \pi i / 3}$ and $\theta=\omega-\bar{\omega}=\sqrt{-3}$. Let $\mathcal{E}$ be the ring $\mathbb{Z}[\omega]$ of Eisenstein integers. An Eisenstein lattice $K$ means an hermitian $\mathcal{E}$-lattice, i.e., a free $\mathcal{E}$-module with an hermitian form $\langle\mid\rangle: K \times K \rightarrow \mathbb{Q}(\omega)$, linear in the first variable and antilinear in the second. We abbreviate $K \otimes_{\varepsilon} \mathbb{C}$ to $K \otimes \mathbb{C}$. If $K$ is nondegenerate then its dual lattice is defined as $K^{*}=\{x \in K \otimes \mathbb{C}:\langle x \mid k\rangle \in$ $\mathcal{E}$ for all $k \in K\}$. The norm $v^{2}$ of $v \in K$ means $\langle v \mid v\rangle$. If $X$ is a subset of a lattice then we write $X^{\perp}$ for the set of lattice vectors orthogonal to it. If $x, y, \ldots$ lie in an $\mathcal{E}$-lattice, then $\langle x, y, \ldots\rangle$ means their $\mathcal{E}$-span.

In the appendices, starting with lemma A.8, the Eisenstein integer $\psi=1-3 \bar{\omega}$ plays an important role.

2.2. Complex hyperbolic space. We call an Eisenstein lattice $K$ Lorentzian if it has signature $(n, 1)$. In that case we let $\mathbb{B}(K) \subseteq P(K \otimes \mathbb{C})$ denote the set of complex lines of negative norm in $K \otimes \mathbb{C}$. Topologically it is a complex ball of dimension $n$. It has a natural metric called the Bergman metric, and is sometimes called complex hyperbolic space. If it is clear what lattice we mean then we sometimes write $\mathbb{B}^{n}$ in place of $\mathbb{B}(K)$. In particular, $\mathbb{B}^{13}$ will always mean $\mathbb{B}(L)$ for the lattice $L$ defined in section 2.4 below. An inclusion of Lorentzian lattices induces an inclusion of their complex balls. If this inclusion has codimension 1 then we call the smaller ball a hyperplane.

Any vector $v$ of negative norm in $K \otimes \mathbb{C}$ determines a point $\mathbb{C} v$ in $\mathbb{B}(K)$. Often we use the same symbol for the point and the vector. The distance between two points of $\mathbb{B}(K)$ is given by

$$
d(v, w)=\cosh ^{-1} \sqrt{\frac{|\langle v \mid w\rangle|^{2}}{v^{2} w^{2}}}
$$

where $v$ and $w$ are two negative norm vectors of $K \otimes \mathbb{C}$. Similarly, if $v, s \in K \otimes \mathbb{C}$ have negative and positive norm respectively, then

$$
d\left(v, \mathbb{B}\left(s^{\perp}\right)\right)=\sinh ^{-1} \sqrt{-\frac{|\langle v \mid s\rangle|^{2}}{v^{2} s^{2}}}
$$

When $s$ is a root (see section 2.5 below) we usually write $s^{\perp}$ in place of $\mathbb{B}\left(s^{\perp}\right)$ when it is clear that we mean this rather than the orthogonal complement in the lattice. Formulas (2.1) and (2.2) differ from those in Go by an unimportant factor of 2 .

The boundary $\partial \mathbb{B}(K)$ of $\mathbb{B}(K)$ in $P(K \otimes \mathbb{C})$ is a real $(2 n-1)$-sphere. Its points are the projectivizations of null vectors, meaning non-zero vectors in $K \otimes \mathbb{C}$ of norm 0 . Given such a vector $\rho$, we define a sort of distance-to- $\rho$ function on $\mathbb{B}(K)$, called the height:

$$
\operatorname{ht}_{\rho}(v)=-\frac{|\langle v \mid \rho\rangle|^{2}}{v^{2}}
$$


This is invariant under scaling $v$, so it descends to a function on $\mathbb{B}(K)$. We will say that one point of $\mathbb{B}(K)$ is closer to $\rho$ than another point is, if its value of ht $\rho$ is smaller. The horosphere centered at $\rho$, of height $h$, means the set of $v \in \mathbb{B}(K)$ with $h_{\rho}(v)=h$. We define open and closed horoballs the same way, replacing $=$ by $<$ and $\leq$. Scaling $\rho$ by $\lambda \in \mathbb{C}$ scales the function ht $\rho$ by $|\lambda|^{2}$. But for us there will be a canonical normalization, because we will always take $\rho$ to be a primitive lattice vector. Under this condition, the only allowed scaling of $\rho$ is by sixth roots of unity, which does not affect the height function. We call the point of $\partial \mathbb{B}(K)$ represented by a primitive lattice vector a cusp, and for convenience we also call that vector itself a cusp. We write $\overline{\mathbb{B}(K)}$ for the topological closure $\mathbb{B}(K) \cup \partial \mathbb{B}(K)$ of $\mathbb{B}(K)$ in $P(K \otimes \mathbb{C})$.

2.3. Geodesics and totally geodesic triangles. Continuing to take $K$ as above, suppose $v, w \in K \otimes \mathbb{C}$ represent points of $\overline{\mathbb{B}(K)}$. Then the geodesic joining them may be described as follows. First we rescale one or both of $v, w$ so that $\langle v \mid w\rangle \in(-\infty, 0]$; this inner product will be negative unless $v$ and $w$ both represent the same point of $\partial \mathbb{B}(K)$. After this rescaling, the geodesic is the image in projective space of the real line segment in $K \otimes \mathbb{C}$ joining the vectors. If we have in mind a third point of $\overline{\mathbb{B}(K)}$, represented by $x \in K \otimes \mathbb{C}$, then there may or may not be a totally geodesic surface in $\overline{\mathbb{B}(K)}$ whose closure contains all three points. If there is one then we write $\triangle v w x$ for their convex hull. There are two cases in which we use this notation. One is when $v, w, x$ all lie in some complex 2-space in $K \otimes \mathbb{C}$. Then they lie in (the closure of) some $\mathbb{B}^{1}$. In this case we call $\triangle v w x$ a complex triangle.

The other case occurs when it is possible to scale $v, w, x$ so that their pairwise inner products lie in $(-\infty, 0]$. Suppose this has been accomplished. The image in $\mathbb{B}(K)$ of the set of negative-norm vectors in the real span of $v, w, x$ is a totally geodesic copy of the real hyperbolic plane. In this case the projectivization of the convex hull of $v, w$ and $x$ in $K \otimes \mathbb{C}$ is the convex hull of their images in $\overline{\mathbb{B}(K)}$. In this case we call $\triangle v w x$ a totally real triangle, and don't usually distinguish between the triangle in $K \otimes \mathbb{C}$ and its image in $\overline{\mathbb{B}(K)}$.

For us this situation occurs as follows. Let $b$ be a point of $\overline{\mathbb{B}(K)}, H$ be a hyperplane in $\mathbb{B}(K), p$ be the point of $H$ closest to $b$, and $q$ be another point of $H$. Using the same letters for vectors representing these points, and $s$ for a positivenorm vector orthogonal to $H$, we first scale $b$ so that its inner product with $s$ is real, and then scale $q$ so that that its inner product with $b$ lies in $(-\infty, 0]$. Then $p=b-\langle b \mid s\rangle s / s^{2}$ has inner product $b^{2}-\langle b \mid s\rangle^{2} / s^{2} \leq 0$ with $b$, and by $s \perp q$ we have $\langle p \mid q\rangle=\langle b \mid q\rangle \leq 0$. So $\triangle b p q$ is a totally real triangle.

2.4. The $P^{2} \mathbb{F}_{3}$ model of the lattice $L$. Now we set up an explicit model for the $\mathcal{E}$-lattice $L$ that governs everything in this paper, called the $P^{2} \mathbb{F}_{3}$ model. It was implicit in Ba1 (eq. 25 in proof of prop. 6.1) and was defined explicitly in A3. Most of our computations will be done in this coordinate system. See section 2.7 for an alternate model of $L$, the Leech model.

We write elements of $\mathbb{C}^{14}$ as vectors $x=\left(x_{0} ; x_{1}, \cdots, x_{13}\right)$ and use the standard hermitian form of signature $(13,1)$ on $\mathbb{C}^{14}$, namely

$$
\langle x \mid y\rangle=-x_{0} \bar{y}_{0}+x_{1} \bar{y}_{1}+\cdots+x_{13} \bar{y}_{13} .
$$

We index the last 13 coordinates by the points of $P^{2} \mathbb{F}_{3} . L$ consists of all vectors $x \in \mathcal{E}^{14}$ such that $x_{0} \equiv x_{1}+\cdots+x_{13} \bmod \theta$ and that $\left(x_{1}, \ldots, x_{13}\right)$, modulo $\theta$, is 
an element of the "line code", meaning the 7 -dimensional subspace of $\mathbb{F}_{3}^{13}$ spanned by the characteristic functions of the lines of $P^{2} \mathbb{F}_{3}$. The elements of the line code are tabulated in tables 2 and 3 of [A3]; the explicit list is not needed in this paper. We write $\Gamma$ for Aut $L$. It contains the simple group $L_{3}(3)=\operatorname{PGL}_{3}\left(\mathbb{F}_{3}\right)$, acting by permuting the points of $P^{2} \mathbb{F}_{3}$ in the obvious way. It also contains the group $3^{13}=(\mathbb{Z} / 3)^{13}$, acting by multiplying the last 13 coordinates by cube roots of unity.

An important property of $L$ is that it equals $\theta \cdot L^{*}$. The proof amounts to checking that all inner products are divisible by $\theta$ and that $\operatorname{det} L=-3^{7}$. The first part is easy, using the point- and line-roots from section 2.6. The second part follows from the fact that $L /(\theta \mathcal{E})^{14} \cong \mathbb{F}_{3}^{7}$.

2.5. Roots, mirrors and the hyperplane arrangement $\mathcal{H}$. A root of $L$ means a lattice vector of norm 3. What makes roots special is that their "triflections" preserve $L$. That is, supposing $s$ is a root, we define $R_{s}$ as the automorphism of $L$ that fixes $s^{\perp}$ pointwise and multiplies $s$ by the cube root of unity $\omega$. A formula is

$$
R_{s}: x \mapsto x-(1-\omega) \frac{\langle x \mid s\rangle}{s^{2}} s
$$

One can show that this isometry of $L \otimes \mathbb{C}$ preserves $L$. (The key is that all inner products in $L$ are divisible by $\theta$.) It is called the $\omega$-reflection in $s$. Replacing $\omega$ by $\bar{\omega}$ gives the $\bar{\omega}$-reflection, which is also $R_{s}^{-1}$. Both of these isometries are complex reflections of order 3 , sometimes called triflections. It is known that $\Gamma$ is generated by the triflections in the roots of $L$; see [Ba1] or [A3].

As the fixed-point set of the reflection $R_{s}$, the hyperplane $\mathbb{B}\left(s^{\perp}\right) \subseteq \mathbb{B}(L)$ is called the mirror of $s$. In this paper we use the word mirror exclusively for hyperplanes orthogonal to roots. The union of all mirrors is called $\mathcal{H}$. This hyperplane arrangement is central to the paper, since our goal is to study the orbifold fundamental group of $\left(\mathbb{B}^{13}-\mathcal{H}\right) / P \Gamma$.

2.6. Point-roots, line-roots, 13-points and 26-points. It is possible to number the points and lines of $P^{2} \mathbb{F}_{3}$ by the numbers $1, \ldots, 13$, such that the $j$ th line is the set of points $\{j, j+1, j+3, j+9\}$. Here the indices should be read modulo 13 . We adopt this numbering for the following important roots of $L$. First, the 13 pointroots $p_{i}$ are the vectors $p_{1}=(0 ; \theta, 0, \ldots, 0), \ldots, p_{13}=(0 ; 0, \ldots, 0, \theta)$. And second, for $j=1, \ldots, 13$, the line-root $l_{j}$ is the vector of the form $\left(1 ; 1^{4}, 0^{9}\right)$ with 1 's along the $j$ th line of $P^{2} \mathbb{F}_{3}$. Explicitly,

$$
\begin{array}{ll}
l_{1}=(1 ; 1101000001000) & l_{8}=(1 ; 0001000110100) \\
l_{2}=(1 ; 0110100000100) & l_{9}=(1 ; 0000100011010) \\
l_{3}=(1 ; 0011010000010) & l_{10}=(1 ; 0000010001101) \\
l_{4}=(1 ; 0001101000001) & l_{11}=(1 ; 1000001000110) \\
l_{5}=(1 ; 1000110100000) & l_{12}=(1 ; 0100000100011) \\
l_{6}=(1 ; 0100011010000) & l_{13}=(1 ; 1010000010001) \\
l_{7}=(1 ; 0010001101000) &
\end{array}
$$

We speak of a point-root $p_{i}$ and a line-root $l_{j}$ as being incident when the corresponding point and line of $P^{2} \mathbb{F}_{3}$ are. For example, $\left\langle p_{i} \mid l_{j}\right\rangle=\theta$ or 0 according to whether or not $p_{i}$ and $l_{j}$ are incident. Also, distinct point-roots are orthogonal, as are distinct line-roots.

There is some obvious symmetry preserving this configuration of roots: $L_{3}(3)$ sends point-roots to point-roots and line-roots to line-roots. But there is additional symmetry. From a correlation of $P^{2} \mathbb{F}_{3}$, i.e., an incidence-preserving exchange of 
points with lines, one can construct an isometry of $L$ that sends the point-roots to line-roots and the line-roots to negated point-roots. (We give such an isometry explicitly in the proof of lemma A.13) Together with scalars and $L_{3}(3)$, this generates a subgroup $\left(6 \times L_{3}(3)\right) \cdot 2$ of $\Gamma$, whose image in $P \Gamma$ is $L_{3}(3): 2$. Here we are using ATLAS notation [ATLAS: a group has "structure $A . B$ " if it has a normal subgroup isomorphic to a group $A$, with quotient isomorphic to a group $B$. If the extension splits then one can indicate this by writing $A: B$ instead. If it does not split then one can write $A \cdot B$. We are also using another ATLAS notation: writing $n$ to indicate a cyclic group of order $n$.

We define the point- and line-mirrors to be the mirrors of the point- and lineroots. The 13 point-mirrors are mutually orthogonal and intersect at a single point of $\mathbb{B}^{13}$, represented by

$$
p_{\infty}=\left(\bar{\theta} ; 0^{13}\right) .
$$

For lack of a better name we call it a 13-point to indicate the 13 mirrors passing through it. (It is easy to see that there are no mirrors through it except the pointmirrors.) We apply the same term to its $\Gamma$-translates, such as the intersection point of the 13 line-mirrors, represented by

$$
l_{\infty}=\left(4 ; 1^{13}\right) .
$$

We summarize the inner product information about $p_{\infty}, p_{1}, \ldots, p_{13}, l_{\infty}, l_{1}, \ldots, l_{13}$ as follows. First, all have norm 3 except for $p_{\infty}$ and $l_{\infty}$, which have norm -3 . Second, if $i \neq j$ then $p_{i} \perp p_{j}$ and $l_{i} \perp l_{j}$. Third, $\left\langle p_{\infty} \mid l_{\infty}\right\rangle=4 \theta$. Finally, if $i$ and $j$ are not both $\infty$, then $\left\langle p_{i} \mid l_{j}\right\rangle$ is $\theta$ or 0 according to whether or not $p_{i}$ and $l_{j}$ are incident. Here we are regarding $p_{\infty}$ as "incident" to every $l_{j}$, and $l_{\infty}$ as "incident" to every $p_{i}$.

We define the basepoint $\tau$ used in theorem 1.2 as the midpoint of the segment joining $p_{\infty}$ and $l_{\infty}$. It is represented by the vector

$$
\tau=l_{\infty}+i p_{\infty}=\left(4+\sqrt{3} ; 1^{13}\right)
$$

of norm $-6-8 \sqrt{3}$, and is the unique fixed point of $L_{3}(3): 2 \subseteq P \Gamma$. It is known that the mirrors closest to $\tau$ are exactly the 26 point- and line-mirrors. (See Ba1, prop. 1.2], where $\tau$ was called $\bar{\rho}$, or lemma A.5 in this paper.) Two consequences of this are that no mirrors pass through $\tau$, and that $L_{3}(3): 2$ is the full $P \Gamma$-stabilizer of $\tau$. For lack of a better name, we call $\tau$ a 26-point to indicate these 26 nearest mirrors. We use the same language for its $\Gamma$-translates.

2.7. The Leech model of $L$. By the Leech lattice we mean what might better be called the complex Leech lattice $\Lambda$. It is a 12 -dimensional positive definite $\mathcal{E}$ lattice, described in detail in $\mathrm{W}$. At the smallest scale at which it is integral as an $\mathcal{E}$-lattice, it has minimal norm 6 and satisfies $\Lambda=\theta \cdot \Lambda^{*}$ and $\operatorname{det} \Lambda=3^{6}$. Also see $[\mathrm{W}$ ] for a thorough study of Aut $\Lambda$, which is the universal central extension 6. Suz of Suzuki's sporadic finite simple group $S u z$. It will be important for us that $6 \cdot S u z$ acts transitively on the lattice vectors of norm 6 resp. 9 . We will postpone an explicit description of $\Lambda$ until the second half of appendix A, because we won't need it until then.

The Leech model of $L$ is useful when one has in mind a Leech cusp (see below). It is: $L \cong \Lambda \oplus\left(\begin{array}{cc}0 & \bar{\theta} \\ \theta & 0\end{array}\right)$. This means that we write lattice vectors as $(x ; y, z)$ where 
$x \in \Lambda$ and $y, z \in \mathcal{E}$, with the inner product given by

$$
\left\langle(x ; y, z) \mid\left(x^{\prime} ; y^{\prime}, z^{\prime}\right)\right\rangle=\left\langle x \mid x^{\prime}\right\rangle+\left(\begin{array}{ll}
y & z
\end{array}\right)\left(\begin{array}{cc}
0 & \bar{\theta} \\
\theta & 0
\end{array}\right)\left(\begin{array}{c}
\bar{y}^{\prime} \\
\bar{z}^{\prime}
\end{array}\right)
$$

This model of $L$ was introduced in [A1], and proven to be isometric to the $P^{2} \mathbb{F}_{3}$ model in [Ba1, Lemma 2.6]. The Leech cusp $\rho=(0 ; 0,1)$ is distinguished, and the mirrors nearest it can be conveniently parameterized as explained in section 2.8 .

2.8. Leech cusps and Leech roots. Earlier we declared that if $v \in L$ is a primitive null vector then we call it (or the point of $\partial \mathbb{B}^{13}$ it represents) a cusp. We refine this to "Leech cusp" in the special case that $v^{\perp} /\langle v\rangle$ is isometric to the Leech lattice. An example which will play a key role in this paper is the primitive null vector $\rho$ defined as $(0 ; 0,1)$ in the Leech model. For background we remark that there are five $\Gamma$-orbits on primitive null vectors $v \in L$, corresponding to the five possibilities for the isometry class of $v^{\perp} /\langle v\rangle$, which are the "Eisenstein Niemeier lattices". (See Lemma 2 and Theorem 4 of $\mathrm{A3}$.) In the $P^{2} \mathbb{F}_{3}$ model it is harder to find a Leech cusp, but an example is $\rho=(3 \omega-1 ;-1, \ldots,-1)$. (Proof sketch following the proof of theorem 1 in [A3: $\rho^{\perp} /\langle\rho\rangle$ is an Eisenstein Niemeier lattice, and its isometry group obviously contains $L_{3}(3)$. By [A3, Thm. 4] the Leech lattice is the only Eisenstein Niemeier lattice whose symmetry group contains $L_{3}(3)$.) We hope that the simultaneous use of $\rho$ for $(0 ; 0,1)$ in the Leech model and $(3 \omega-1 ;-1, \ldots,-1)$ in the $P^{2} \mathbb{F}_{3}$ model is helpful rather than confusing: we think of the models as two ways to describe a single lattice. In lemma A.10 we complete the identification of the two models by explicitly writing down the point- and line-roots in the Leech model.

The following description of vectors $s \in L \otimes \mathbb{C}$ not orthogonal to $\rho$ is very useful: every such $s$ can be written uniquely in the form

$$
s=\left(\sigma ; m, \frac{\theta}{\bar{m}}\left(\frac{\sigma^{2}-N}{6}+\nu\right)\right)
$$

where $\sigma \in \Lambda \otimes \mathbb{C}, m \in \mathbb{C}-\{0\}, N$ is the norm $s^{2}$, and $\nu$ is purely imaginary. Restricting the first component to $\Lambda$ and the others to $\mathcal{E}$ gives the elements of $L-\rho^{\perp}$. Further restricting $N$ to 3 gives the roots of $L$, and finally restricting $m$ to 1 gives the Leech roots (see equation (2.8) below). We remark that scaling $s$ by $\lambda \in \mathbb{C}-\{0\}$ scales $\sigma$ and $m$ by $\lambda$, and $N$ and $\nu$ by $|\lambda|^{2}$.

The elaborate form of the last coordinate in (2.6) allows the following interpretation of $\left\langle s \mid s^{\prime}\right\rangle$, where $s$ is from (2.6) and similarly for $s^{\prime}$ :

$$
\begin{aligned}
\left\langle s \mid s^{\prime}\right\rangle=m \bar{m}^{\prime}\left[\frac { 1 } { 2 } \left(\frac{N^{\prime}}{\left|m^{\prime}\right|^{2}}+\right.\right. & \left.\frac{N}{|m|^{2}}-\left(\frac{\sigma}{m}-\frac{\sigma^{\prime}}{m^{\prime}}\right)^{2}\right) \\
& \left.+\operatorname{Im}\left\langle\frac{\sigma}{m} \mid \frac{\sigma^{\prime}}{m^{\prime}}\right\rangle+3\left(\frac{\nu^{\prime}}{\left|m^{\prime}\right|^{2}}-\frac{\nu}{|m|^{2}}\right)\right] .
\end{aligned}
$$

The content of this is that $\left\langle s \mid s^{\prime}\right\rangle$ is governed by the relative positions of $\sigma / m$ and $\sigma^{\prime} / m^{\prime}$ in $\Lambda \otimes \mathbb{C}$, with $\nu$ and $\nu^{\prime}$ influencing only the imaginary part of the bracketed term. (Caution: we are using the convention that the imaginary part of a complex number is imaginary; for example $\operatorname{Im} \theta$ is $\theta$ rather than $\sqrt{3}$.) One proves the formula by writing out $\left\langle s \mid s^{\prime}\right\rangle$, completing the square and patiently rearranging.

Because the Leech lattice has no vectors of norm $<6$, a Leech cusp $\rho \in \partial \mathbb{B}^{13}$ lies in (the closures of) no mirrors. The mirrors which come closest to it are called 
first-shell mirrors, and the second- and third-shell mirrors are defined similarly. We use the same language for their corresponding roots. When the Leech cusp is $\rho$, then $s$ is a first-, second- or third-shell root if $|\langle\rho \mid s\rangle|^{2}$ is 3,9 or 12 respectively. It will be useful to fix a scaling of the first-shell roots: a Leech root (with respect to $\rho$ ) means a root $s$ with $\langle\rho \mid s\rangle=\theta$, and we call its mirror a Leech mirror.

In the Leech model, the Leech roots (with respect to $\rho$ ) are the vectors

$$
s=\left(\sigma ; 1, \theta\left(\frac{\sigma^{2}-3}{6}+\nu\right)\right)
$$

with $\sigma \in \Lambda$ and $\nu \in \frac{1}{\theta} \mathbb{Z}+\frac{1}{2 \theta}$ if $\sigma^{2}$ is divisible by 6 , or $\nu \in \frac{1}{\theta} \mathbb{Z}$ otherwise. This is a specialization of (2.6). We note that if $(\sigma ; 1, \alpha)$ is a Leech root, then the Leech roots of the form $(\sigma ; 1, *)$ are $(\sigma ; 1, \alpha+n)$ as $n$ runs over $\mathbb{Z}$. In the $P^{2} \mathbb{F}_{3}$ model there is no simple formula for the Leech roots, but (with respect to $\rho$ ) the point-roots are examples of Leech roots, and the line-roots are examples of second-shell roots. For later use, we record the inner products

$$
\left\langle\rho \mid p_{i}\right\rangle=\theta \text { and }\left\langle\rho \mid l_{i}\right\rangle=3 \bar{\omega} \text { for } i=1, \cdots, 13 .
$$

In section 3 we will choose a closed horoball $A$ centered at $\rho$ and disjoint from $\mathcal{H}$, and choose a point $a \in A$. We will speak of "meridians" to refer to certain elements $M_{a, A, H}$ of the orbifold fundamental group (see below) of $\left(\mathbb{B}^{13}-\mathcal{H}\right) / P \Gamma$, or their underlying paths $\mu_{a, A, H}$. The definition of a meridian involves a choice of a mirror $H$; we will call it a Leech meridian if $H$ is a Leech mirror.

2.9. Meridians and the orbifold fundamental group. The orbifold fundamental group $G_{b}=\pi_{1}^{\text {orb }}\left(\left(\mathbb{B}^{13}-\mathcal{H}\right) / P \Gamma, b\right)$ depends on the choice of a base point $b \in \mathbb{B}^{13}-\mathcal{H}$. It means the following set of equivalence classes of pairs $(\gamma, g)$, where $g \in P \Gamma$ and $\gamma$ is a path in $\mathbb{B}^{13}-\mathcal{H}$ from $b$ to $g(b)$. One such pair is equivalent to another one $\left(\gamma^{\prime}, g^{\prime}\right)$ if $g=g^{\prime}$ and $\gamma$ and $\gamma^{\prime}$ are homotopic in $\mathbb{B}^{13}-\mathcal{H}$, rel endpoints. The group operation is $(\gamma, g) \cdot\left(\gamma^{\prime}, g^{\prime}\right)=\left(\gamma\right.$ followed by $\left.g \circ \gamma^{\prime}, g g^{\prime}\right)$. Inversion is given by $(\gamma, g)^{-1}=\left(g^{-1} \circ\right.$ reverse $\left.(\gamma), g^{-1}\right)$. Projection of $(\gamma, g)$ to $g$ defines a homomorphism $G_{b} \rightarrow P \Gamma$. It is surjective because $\mathbb{B}^{13}-\mathcal{H}$ is path-connected. The kernel is obviously $\pi_{1}\left(\mathbb{B}^{13}-\mathcal{H}, b\right)$, yielding the exact sequence

$$
1 \rightarrow \pi_{1}\left(\mathbb{B}^{13}-\mathcal{H}, b\right) \rightarrow G_{b} \rightarrow P \Gamma \rightarrow 1
$$

The elements of $G_{b}$ we will use are the "meridians" $M_{b, H}$ and $M_{b, q, H}$, where $H$ is a mirror and $q$ is a point of $H$. These are defined in section 3 Their underlying paths are written $\mu_{b, H}$ and $\mu_{b, q, H}$ and are also called meridians. The meridians associated to the point- and line-mirrors are called the point- and line-meridians. The main theorem of this paper is that $G_{\tau}$ is generated by these 26 meridians, when the basepoint $\tau$ is the 26-point from section 2.6. There are also "fat basepoint" versions of meridians: $M_{a, A, H}, M_{a, A, q, H}, \mu_{a, A, H}$ and $\mu_{a, A, q, H}$ with $A$ a horoball centered at a Leech cusp, small enough to miss $\mathcal{H}$, and $a$ is a point of $A$. These are also defined in section 3. Of the meridians of this sort, we mostly use those with $H$ a Leech mirror, which we call Leech meridians. We also speak of second-shell meridians, with the corresponding meaning.

In any $\mu_{\ldots}$ or $M_{\ldots}$, we write just $s$ instead of $s^{\perp}$ in the subscript when the hyperplane is the mirror $s^{\perp}$ of a root. For example, $\mu_{b, s}$ rather than $\mu_{b, s^{\perp}}$. 


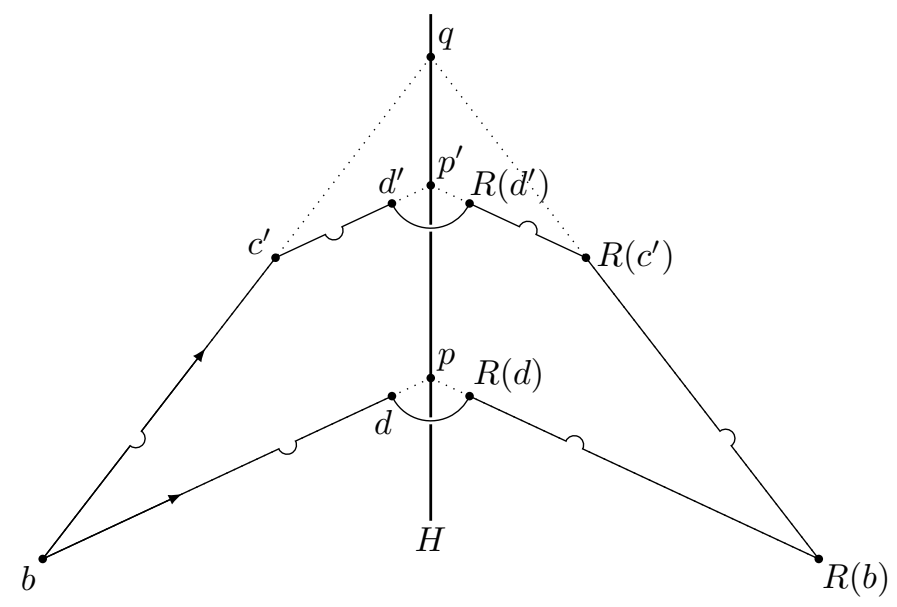

Figure 3.1. The meridians $\mu_{b, q, H}$ (top) and $\mu_{b, H}$ (bottom) go left to right from $b$ to $R(b)$. Here $b$ is the basepoint, $H$ is a mirror, $R$ is its $\omega$-reflection, $q \in H$, and $p$ is the point of $H$ nearest $b$. The semicircular arcs in the paths indicate possible diversions around points of $\mathcal{H}$.

\section{Meridians AND homotopies BetWeen them}

At its heart this paper consists of explicit manipulations of paths in $\mathbb{B}^{13}-\mathcal{H}$. In this section we describe the most important paths and manipulations. For concreteness we work only with our particular arrangement $\mathcal{H}$ in $\mathbb{B}^{13}$, but it will be obvious that everything works equally well in the generality of $\mathrm{AB}$. (In particular, for the mirror arrangements of finite complex reflection groups.) The ideas are simple, and we hope the following summary will enable the reader to skip the technical details.

Our basic path is called a "meridian" and written $\mu_{b, q, H}$. Here $b$ is a point of $\mathbb{B}^{13}-\mathcal{H}, H$ is a mirror, and $q$ is a point of $H$. The path begins at $b$ and travels along a geodesic towards $q$, stopping near it. Next it travels directly toward $H$, stopping very near it. Then it travels $2 \pi / 3$ of the way around $H$, on a positively oriented circular arc. Finally it "returns" by retracing the paths in the first two steps of the construction (or rather their images under the $\omega$-reflection in $H$ ). See figure 3.1. The term "meridian" is from knot theory: we think of the image of $\mathcal{H}$ in $\mathbb{B}^{13} / P \Gamma$ as a sort of knot. "Meridian" indicates that (the image of) $\mu_{b, q, H}$ is a loop, freely homotopic to a circle around (the image of) $H$.

We abbreviate the notation to $\mu_{b, H}$ when $q$ is the point of $H$ nearest $b$. These are the meridians considered in our earlier paper $\mathrm{AB}$.

Lemmas 3.2 and 3.6 are the main results of this section. The conceptual content of lemma 3.6 is the following. Consider the first turning point of $\mu_{b, q, H}$, which we took to be near $q$ (called $c^{\prime}$ in figure 3.1). If we used a point slightly further from $q$ in place of $c^{\prime}$, then we would obtain a homotopic path. If we gradually moved the turning point all the way back to $b$, and all the intermediate paths missed $\mathcal{H}$, then we would have a homotopy from $\mu_{b, q, H}$ to $\mu_{b, H}$. Lemma 3.6 shows that this applies if $\triangle b p q$ misses $\mathcal{H}$ except at the obvious intersection points. Here $p$ is the point of $H$ closest to $b$. 
The conceptual content of lemma 3.2 is the following. Consider a second basepoint $b^{\prime}$, and join $b$ to it by some path $b_{t \in[0,1]}$ that misses $\mathcal{H}$. One expects that under reasonable hypotheses, the family of paths $\mu_{b_{t}, q, H}$ will form a homotopy between $\mu_{b, q, H}$ and $\mu_{b^{\prime}, q, H}$. Lemma 3.2 gives sufficient conditions for this: first, the geodesics $\overline{b_{t} q}$ should miss $\mathcal{H}$ (except at $q$ ), and second, $H$ should be orthogonal to all other mirrors through $q$.

Informally we think of the meridians $\mu_{b, q, H}$ and $\mu_{b, H}$ as elements of the orbifold fundamental group $G_{b}=\pi_{1}^{\text {orb }}\left(\left(\mathbb{B}^{13}-\mathcal{H}\right) / P \Gamma, b\right)$. But strictly speaking, an element of this group is an ordered pair (see section 2.9). So we also say "meridian" for the ordered pairs $\left(\mu_{b, q, H}, R_{H}\right)$ and $\left(\mu_{b, H}, R_{H}\right)$, where $R_{H}$ is the $\omega$-reflection with mirror $H$. We write $M_{b, q, H}$ and $M_{b, H}$ for them.

In our applications the hyperplane is always the mirror of a root $s$. As mentioned in section 2.9, when writing $\mu \ldots$ or $M \ldots$ we will write just $s$ in the subscript rather than $s^{\perp}$, when $s$ is a root and the hyperplane is its mirror. For example, $\mu_{b, s}$ rather than $\mu_{b, s^{\perp}}$.

Our informal statements of lemmas 3.2 and 3.6 are so natural, even obvious, that the reader may wonder what else there is to say. There are two things to fuss over. First, the basepoint might not be in general position with respect to the hyperplanes. So the proper definition of $\mu_{b, q, H}$ must include detours around any hyperplanes met by the version of $\mu_{b, q, H}$ described above. Then it requires work even to show that the resulting homotopy class is well-defined (lemma 3.1). Second, we would like to use the Leech cusp $\rho$ as a sort of basepoint, even though $\rho$ is not a point of $\mathbb{B}^{13}$. We accomplish this by using the closed horoball $A$ centered at $\rho$ and disjoint from $\mathcal{H}$, and a point $a \in A$. Informally we think of $\rho$ as the basepoint, but $a$ is the official basepoint. In the end one can work with the resulting meridians just like the ones above. But the notation acquires an extra subscript: $\mu_{a, A, q, H}, \mu_{a, A, H}$, $M_{a, A, q, H}$ and $M_{a, A, H}$. Finally, to realize that lemma 3.2 is not a mere tautology, notice the role played by the technical assumption: $H$ is orthogonal to all other mirrors through $q$.

Now we begin the technical content of the section. For $b, c \in \overline{\mathbb{B}^{13}}$ we write $\overline{b c}$ for the geodesic segment from $b$ to $c$. Now suppose $b, c \notin \mathcal{H}$. It may happen that $\overline{b c}$ meets $\mathcal{H}$, so we define a perturbation $\widetilde{b c}$ of $\overline{b c}$ in the obvious way. The notation may be pronounced " $b$ dodge $c$ " or " $b$ detour $c$ ", and the precise definition is the following. We write $\overline{b c}^{\mathbb{C}}$ for the complex line containing $\overline{b c}$. By the local finiteness of the mirror arrangement, $\overline{b c} \mathbb{C} \cap \mathcal{H}$ is a discrete set. Consider the path got from $\overline{b c}$ by using positively oriented semicircular detours in $\overline{b c} \mathbb{C}$, around the points of $\overline{b c} \cap \mathcal{H}$, in place of the corresponding segments of $\overline{b c}$. By $\widetilde{b c}$ we mean this path, with the radius of these detours taken to be small enough. (This means: small enough for the construction to make sense and the resulting homotopy class in $\mathbb{B}^{13}-\mathcal{H}$, rel endpoints, to be radius-independent.)

Now we define $\mu_{b, H}$ for $b \in \mathbb{B}^{13}-\mathcal{H}$ and $H$ a hyperplane of $\mathcal{H}$. See the bottom path in figure 3.1 Write $p$ for the point of $H$ nearest $b$ and $R$ for the $\omega$-reflection with mirror $H$. Choose an open ball $U$ around $p$, small enough to miss all the mirrors except those through $p$, and choose some $d \in U \cap(\overline{b p}-\{p\})$. From $b \notin \mathcal{H}$ we get $d \notin \mathcal{H}$. Then $\mu_{b, H}$ means $\widetilde{b} \bar{d}$, followed by the positively oriented circular arc in $\overline{b p}^{\mathbb{C}}$ from $d$ to $R(d)$, centered at $p$, followed by $R($ reverse $(\widetilde{b} \bar{d}))$. It is easy to see that the resulting path is independent of our choices of $U$ and $d$, up to homotopy 


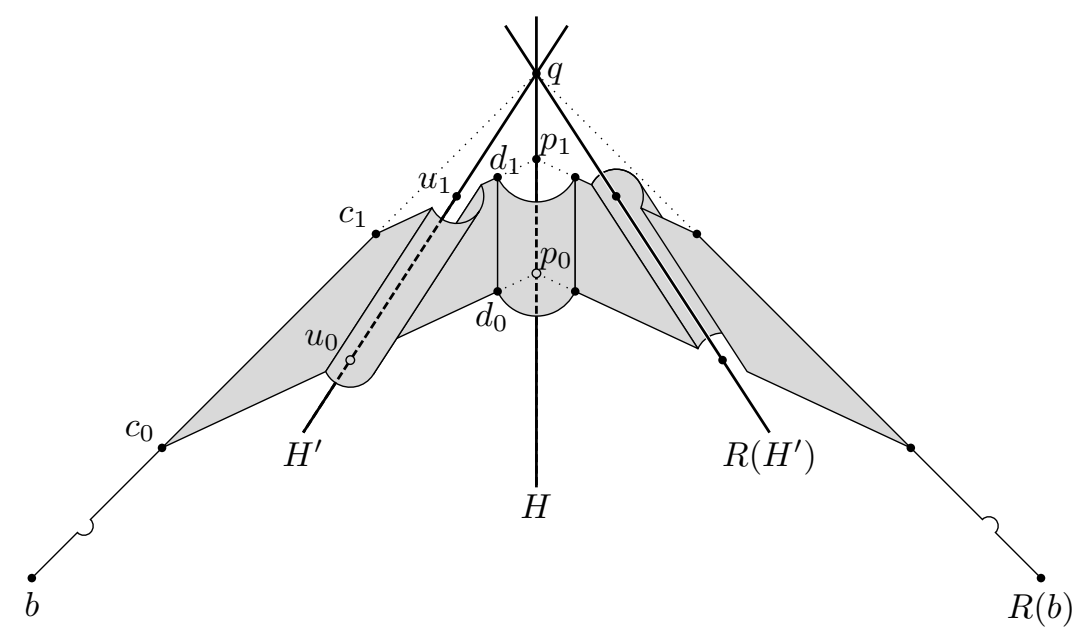

Figure 3.2. The well-definedness of $\mu_{b, q, H}$; see lemma 3.1. The unlabeled points on the right side are the $R$-images of $c_{0}, c_{1}, u_{0}$, $u_{1}, d_{0}$ and $d_{1}$.

rel endpoints in $\mathbb{B}^{13}-\mathcal{H}$. As mentioned above, $M_{b, H}$ means the element $\left(\mu_{b, H}, R\right)$ of the orbifold fundamental group, and is also called a meridian. Although we don't need it, we mention for background that $M_{b, H}^{3}$ is the loop in $\mathbb{B}^{13}-\mathcal{H}$ that we called $\overrightarrow{b H}$ (" $b$ loop $H$ ") in $\underline{\mathrm{AB}}$. It goes from $b$ to $d$, encircles $H$ in $\overline{b p} \mathbb{C}$, and then returns to $b$.

Next we define $\mu_{b, q, H}$, where $q$ is a point of $H$. See the top path in figure 3.1. Choose an open ball $U$ around $q$ that is small enough to miss all the mirrors except those through $q$, and choose some point $c^{\prime} \in U \cap(\overline{b q}-\{q\})$. From $b \notin \mathcal{H}$ we get $c^{\prime} \notin \mathcal{H}$, so $\mu_{c^{\prime}, H}$ is defined. We define $\mu_{b, q, H}$ as $\widehat{b c^{\prime}}$ followed by $\mu_{c^{\prime}, H}$ followed by $R\left(\right.$ reverse $\left(\widetilde{b c^{\prime}}\right)$ ). Figure 3.1 also shows primed versions of the points $p$ and $d$ from the previous paragraph's definition of $\mu_{c^{\prime}, H}$. The next lemma shows that $\mu_{b, q, H}$ is well-defined as a homotopy class. The tricky part is dealing with possible detours in the subpath $\mu_{c^{\prime}, H}$. (For the meridians in this paper, it happens that no such detours occur, in which case the lemma is obvious.) Just as with $M_{b, H}$, we refer to the orbifold fundamental group element $M_{b, q, H}=\left(\mu_{b, q, H}, R\right)$ as a meridian.

Lemma 3.1 (Well-definedness of $\mu_{b, q, H}$ ). The homotopy class of $\mu_{b, q, H}$, rel endpoints, is independent of the choices of $U$ and $c^{\prime}$.

Proof. Suppose $U$ is a ball around $q$ as above, and write $c_{0}, c_{1} \in U \cap(\overline{b q}-\{q\})$ for two candidates for $c^{\prime}$. We choose the subscripts so that $c_{0}$ is further from $q$ than $c_{1}$ is. We will show that the version of $\mu_{b, q, H}$ defined using $c^{\prime}=c_{0}$ is homotopic rel endpoints to the version of $\mu_{b, q, H}$ defined using $c^{\prime}=c_{1}$. The shaded surface in figure 3.2 is the homotopy that we will construct. It is a homotopy from $\mu_{c_{0}, H}$ to $\mu_{c_{1}, H}$ that moves $c_{0}$ along $\overline{c_{0} c_{1}}$. This is enough to build a homotopy between the two versions of $\mu_{b, q, H}$. It follows that the homotopy class of $\mu_{b, q, H}$ is independent of the choice of $c$. It follows that it is also independent of the choice of $U$. 
We parameterize $\overline{c_{0} c_{1}}$ by $t \mapsto c_{t}$ with $t$ varying over $[0,1]$. We write $p_{t}$ for the point of $H$ nearest $c_{t}$. The issue we must deal with is the following. If $\overline{c_{t} p_{t}}-\left\{p_{t}\right\}$ meets $\mathcal{H}$, then varying $t$ will move the intersection points but not eliminate them. This is visible in figure 3.2, where $H^{\prime}$ is a mirror giving rise to such intersections. The method of section 2.3 shows that $c_{0}, p_{0}$ and $q$ span a totally real triangle $\triangle c_{0} p_{0} q$, and also that all the $p_{t}$ lie on the segment $\overline{p_{0} q}$. By the nonpositive curvature of $\mathbb{B}^{13}, p_{0}$ is closer to $q$ than $c_{0}$ is. So $\triangle c_{0} p_{0} q$ lies in $U$. In particular, the mirrors meeting this triangle are among those containing $q$.

It follows that $\mathcal{H}$ meets $\triangle c_{0} p_{0} q$ in the union of finitely many geodesic segments from its vertex $q$ to the opposite edge $\overline{c_{0} p_{0}}$. Now restrict this to the quadrilateral with vertices $c_{0}, c_{1}, p_{1}$ and $p_{0}$. Its intersection with any mirror is either empty or a segment $\overline{u_{0} u_{1}}$, which we may parameterize by $t \mapsto u_{t}$ with $u_{t} \in \overline{c_{t} p_{t}}$ as shown in figure 3.2. If there is more than one such segment then they are disjoint from each other.

Now, any mirror that meets $\overline{p_{0} p_{1}}$ contains all of $\overline{p_{0} p_{1}}$. So we may choose $\varepsilon>0$ such that every mirror meeting the closed $\varepsilon$-neighborhood of $\overline{p_{0} p_{1}}$ contains $\overline{p_{0} p_{1}}$. Now let $D_{t}$ be the closed $\varepsilon$-disk in the complex geodesic ${\overline{c_{t} p_{t}}}^{\mathbb{C}}$, centered at $p_{t}$. The only mirrors it meets are the ones containing $\overline{p_{0} p_{1}}$. All of these mirrors contain $p_{t}$, and therefore contain no other points of $D_{t}$. So $D_{t} \cap \mathcal{H}=\left\{p_{t}\right\}$. Write $d_{t}$ for the point where $\overline{c_{t} p_{t}}$ enters $D_{t}$. Because $D_{t}$ misses $\mathcal{H}$ except at $p_{t}$, we may take $\mu_{c_{t}, H}$ to be $\overline{c_{t}} \overline{d_{t}}$, followed by the positively-oriented arc in $\partial D_{t}$ from $d_{t}$ to $R\left(d_{t}\right)$, followed by $R\left(\right.$ reverse $\left.\left(\overline{c_{t}} \overline{d_{t}}\right)\right)$. The $\partial D_{t}$ portions of these paths vary continuously with $t$, sweeping out the $\frac{1}{3}$-tube in the center of figure 3.2

The shaded surface to the left of this in figure 3.2 is got by modifying the quadrilateral with edges $\overline{d_{1} c_{1}}, \overline{c_{1} c_{0}}, \overline{c_{0} d_{0}}$ and $\left\{d_{t} \mid t \in[0,1]\right\}$. The modification is to replace a strip around each segment $\overline{u_{0} u_{1}}$ (notation as above and in figure 3.2) by a semi-cylindrical strip that dodges $\mathcal{H}$. More precisely, we may take this semicylindrical strip to be a union of positively oriented semicircles $S_{t}$, where $S_{t}$ lies in the complex geodesic $\overline{c_{t} p_{t}} \mathbb{C}$ and has center $u_{t}$ and some small constant radius. Each $S_{t}$ misses $\mathcal{H}$ by reasoning similar to the previous paragraph. Because the $u_{t}$ and the $\overline{c_{t} p_{t}} \mathbb{C}$ vary continuously with $t$, the $S_{t}$ 's do too.

The part of the homotopy on the right side of the figure is the $R$-image of the part on the left. The left, right and middle parts fit together to give the promised homotopy from $\mu_{c_{0}, H}$ to $\mu_{c_{1}, H}$.

Lemma 3.2 (Homotopy between meridians under change of basepoint). Suppose $H$ is a mirror, $q$ is a point of $H$, and that the other mirrors through $q$ are orthogonal to $H$. Suppose $b:[0,1] \rightarrow \mathbb{B}^{13}-\mathcal{H}$ is a path, written $t \mapsto b_{t}$, such that each $\overline{b_{t} q}$ misses $\mathcal{H}$ except at $q$. Then $M_{b_{0}, q, H} \in G_{b_{0}}$ is identified with $M_{b_{1}, q, H} \in G_{b_{1}}$ under the isomorphism of orbifold fundamental groups $G_{b_{0}} \cong G_{b_{1}}$ induced by the path $b$.

Proof. The proof consists mainly of looking at figure 3.3. Let $\varepsilon>0$ be small enough that the only mirrors at distance $\leq \varepsilon$ from $q$ are those that contain $q$. For each $t \in[0,1]$, take the point $c_{t}$ in the definition of $\mu_{b_{t}, q, H}$ to be the point of $\overline{b_{t} q}$ that lies at distance $\varepsilon$ from $q$. (We suppose without loss of generality that $\varepsilon<d\left(q, b_{t}\right)$ for all $t$, so this construction makes sense.) The leftmost region in figure 3.3 represents the union of the paths $\overline{b_{t} c_{t}}$. They all miss $\mathcal{H}$ by hypothesis. The rightmost region is its $R$-image, where $R$ still denotes the $\omega$-reflection in $H$. 


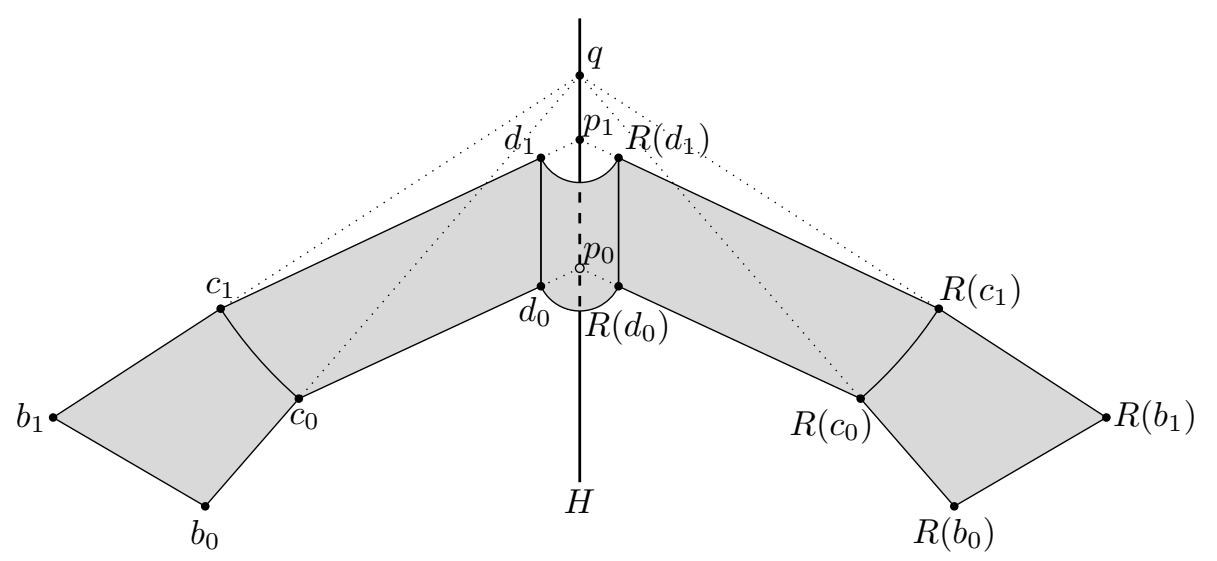

Figure 3.3. Illustration for lemma 3.2 The paths $\mu_{b_{0}, q, H}$ and $\mu_{b_{1}, q, H}$ go left to right across the bottom and top respectively, and the shaded area is a homotopy between them. It moves the basepoint from $b_{0}$ to $b_{1}$.

Now let $p_{t}$ be the point of $H$ closest to $c_{t}$; by the nonpositive curvature of $\mathbb{B}^{13}$, $p_{t}$ is closer to $q$ than $c_{t}$ is. So every point of every geodesic $\overline{c_{t} p_{t}}$ lies at distance $\leq \varepsilon$ from $q$. We claim these geodesics also miss $\mathcal{H}$, except that every $p_{t}$ lies in $H$. Otherwise, some mirror $H^{\prime} \neq H$ would meet some $\overline{c_{t} p_{t}}$. By the construction of $\varepsilon$, $H^{\prime}$ passes through $q$. So our orthogonality hypothesis gives $H^{\prime} \perp H$. Because of this orthogonality, the only way $\overline{c_{t} p_{t}}$ can meet $H^{\prime}$ is by lying entirely in $H^{\prime}$. This would contradict the known fact $c_{t} \notin \mathcal{H}$.

Now let $\varepsilon^{\prime}>0$ be small enough that the closed $\varepsilon^{\prime}$-neighborhood of $\left\{p_{t} \mid t \in\right.$ $[0,1]\}$ misses every mirror except $H$. Call this neighborhood $U$. By definition, the "middle" part of $\mu_{b_{t}, q, H}$ is the path $\mu_{c_{t}, H}$, whose definition involves choosing a point $d_{t}$ very near $p_{t}$. We choose $d_{t}$ to be the point of $\overline{c_{t} p_{t}}$ at distance $\varepsilon^{\prime}$ from $p_{t}$. (We suppose without loss of generality that $\varepsilon^{\prime}<d\left(H, c_{t}\right)$ for every $t$, so this construction makes sense.) The second region in figure 3.3 is the union of the geodesics $\overline{c_{t} d_{t}}$, and the second region from the right is its $R$-image.

The $\frac{1}{3}$-of-a-tube portion of the homotopy in figure 3.3 is the surface swept out by the path $t \mapsto d_{t}$ under the rotations around $H$ by angles in $[0,2 \pi / 3]$. It lies in $U-H=U-\mathcal{H}$, so it misses $\mathcal{H}$. The five pieces of the homotopy fit together to form a homotopy in $\mathbb{B}^{13}-\mathcal{H}$ from $\mu_{b_{0}, q, H}$ to $\mu_{b_{1}, q, H}$, in which $b_{0}$ moves to $b_{1}$ along the path $b$. It follows that the isomorphism $G_{b_{0}} \cong G_{b_{1}}$ induced by this path identifies $\left(\mu_{b_{0}, q, H}, R\right)$ with $\left(\mu_{b_{1}, q, H}, R\right)$. That is, it identifies $M_{b_{0}, q, H}$ with $M_{b_{1}, q, H}$.

Philosophically, we would like to take the Leech cusp $\rho$ as a basepoint for analyzing the orbifold fundamental group of $\left(\mathbb{B}^{13}-\mathcal{H}\right) / P \Gamma$. But we must work around the difficulty that $\rho$ is not a point of $\mathbb{B}^{13}$. To do this we follow the "fat basepoint" strategy of $[\mathrm{AB}$, by choosing a closed horoball $A$ centered at $\rho$ and small enough to miss $\mathcal{H}$. Lemma 4.3 of $[\mathrm{AB}$ ] shows that we may take $A$ to be the closed horoball of any height $<1$. We also choose some point $a$ of $A$.

We continue to suppose that $H$ is a mirror and $R$ is its $\omega$-reflection. Now we take $p$ to be $H$ 's point closest to $\rho$. (See section 2.2 for the meaning of "closest".) 
If the meridian were defined then it would begin with a geodesic (possibly with small detours) from $\rho$ to a point near $p$. This path would pierce $\partial A$ at the point of $\partial A$ nearest to $p$, which we will call $b$. To define the corresponding path based at $a$ we simply replace the segment $\overline{\rho b}$ by $\overline{a b}$. Formally, $\mu_{a, A, H}$ is $\overline{a b}$ followed by $\mu_{b, H}$ followed by $R(\overline{b a})$. See figure 3.4 for a picture: $\mu_{a, A, H}$ travels from left to right across the bottom. The point marked $d$ comes from the definition of $\mu_{b, H}$.

Similarly, if $q$ is any point of $H$ then we define $b^{\prime}$ as the point of $\partial A$ nearest to $q$, and then define $\mu_{a, A, q, H}$ as $\overline{a b^{\prime}}$ followed by $\mu_{b^{\prime}, q, H}$ followed by $R\left(\overline{b^{\prime} a}\right)$. This travels from left to right across the top of figure 3.4. The point marked $c^{\prime}$ comes from the definition of $\mu_{b^{\prime}, q, H}$, and the points marked $d^{\prime}$ and $p^{\prime}$ come from the definition of the subpath $\mu_{c^{\prime}, H}$ of $\mu_{b^{\prime}, q, H}$. Just as for ordinary basepoints, we refer to the orbifold fundamental group elements $M_{a, A, H}=\left(\mu_{a, A, H}, R\right)$ and $M_{a, A, q, H}=\left(\mu_{a, A, q, H}, R\right)$ as meridians.

Remark 3.3. In several places it will clarify an argument to assume "without loss of generality" that $a$ is some particular point of $A$. One should expect that the exact location of $a$ is unimportant: if $a^{\prime}$ is another point of $A$ then $G_{a}$ and $G_{a^{\prime}}$ are canonically isomorphic. One just moves the basepoint from $a$ to $a^{\prime}$ along any path in the simply connected space $A$. But more is needed: this should identify each meridian $M_{a, A, q, H} \in G_{a}$ with $M_{a^{\prime}, A, q, H} \in G_{a^{\prime}}$. It is easy to see that this holds. In fact, arranging for it to be true was the main reason for introducing $A$ into the definition of the meridians. This was necessary because the canonical isomorphism $G_{a} \cong G_{a^{\prime}}$ does not identify every $M_{a, q, H}$ with the corresponding $M_{a^{\prime}, q, H}$.

Remark 3.4. There is a more elegant treatment of fat basepoints in $\mathrm{Be}$, Appendix A]. This treats $A$ itself as the basepoint and avoids mention of $a$. But this approach is not well-suited for moving the basepoint from $a$ to the 26-point $\tau$ (that lies outside $A$ ). We do this in section 6, and it is an essential part of our proof of theorem 1.2 ,

Lemma 3.5 (Homotopies between meridians based "at" a cusp). Suppose $\rho \in \partial \mathbb{B}^{13}$ is a Leech cusp, $A$ is a closed horoball centered at $\rho$ and disjoint from $\mathcal{H}$, and $a$ is a basepoint in $A$. Let $H$ be a mirror, $p$ be the point of $H$ closest to $\rho$, and $q$ be another point of $H$. Our key assumption is that the totally real triangle $\triangle \rho p q$ is disjoint from $\mathcal{H}$ except that $\overline{p q}$ lies in $H$ and that $q$ might lie in additional mirrors. Then $\mu_{a, A, H}$ and $\mu_{a, A, q, H}$ are homotopic rel endpoints in $\mathbb{B}^{13}-\mathcal{H}$, and $M_{a, A, H}=M_{a, A, q, H}$ in $G_{a}$.

Proof. The proof mostly consists of looking at figure 3.4, which uses the notation $b, d, b^{\prime}, c^{\prime}, d^{\prime}, p^{\prime}, R$ from the definitions given above. The paths $\mu_{a, A, q, H}$ and $\mu_{a, A, H}$ are the upper and lower paths around the dark gray region, traversed from left to right. This region indicates the homotopy between them that we will construct.

By hypothesis, for small enough $\varepsilon>0$, the $\varepsilon$-neighborhood of $\overline{p p^{\prime}}$ meets no mirrors except $H$. This neighborhood, which we call $U$, is convex by the nonpositive curvature of $\mathbb{B}^{13}$ (see the remark after Prop. II.2.4 of $[\mathrm{BH}]$ ). By moving $d$ closer to $p$ and $d^{\prime}$ closer to $p^{\prime}$ we may suppose without loss of generality that they lie in $U$. The portion of the homotopy that appears in the figure as $\frac{1}{3}$ of a tube is the surface swept out by $\overline{d d^{\prime}}$ under the rotations around $H$ by angles in $[0,2 \pi / 3]$. It lies in $U$ since $\overline{d d^{\prime}}$ does. It misses $\mathcal{H}$ because it misses $H$, which is the only mirror that meets $U$. The pentagon with vertices $b^{\prime}, c^{\prime}, d^{\prime}, d$ and $b$ misses $\mathcal{H}$ because it 


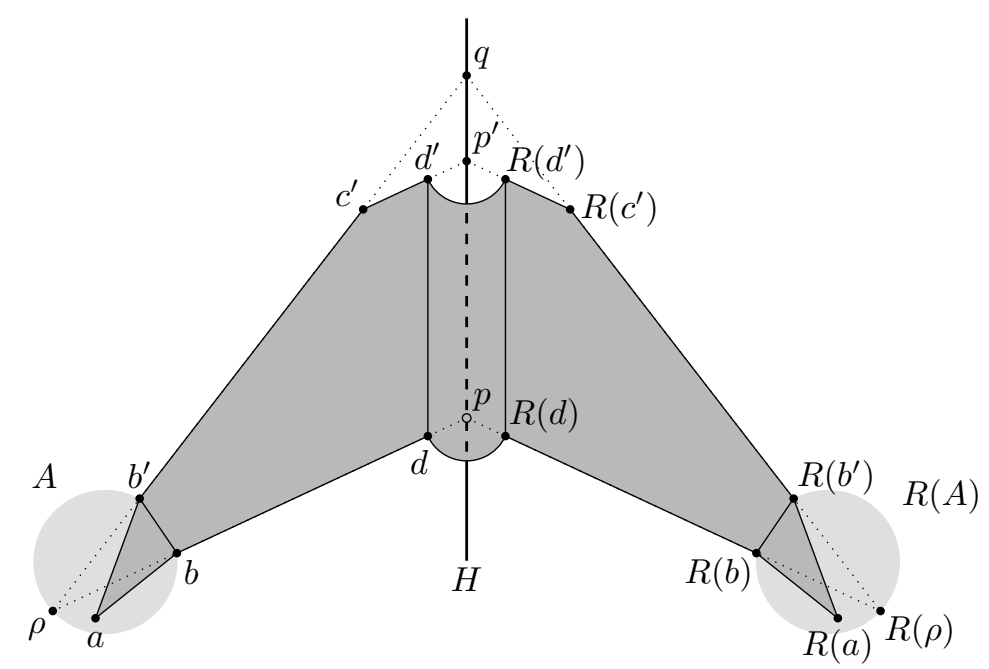

FiguRE 3.4. Illustration of the meridians $\mu_{a, A, H}$, left to right across the bottom, and $\mu_{a, A, q, H}$, left to right across the top. The dark region indicates lemma [3.5]s homotopy between them. The light gray balls are horoballs centered at Leech cusps $\rho$ and $R(\rho)$.

lies in $\triangle \rho p q-\overline{p q}$. (Recall from section 2.3 that $\triangle \rho p q$ is totally geodesic.) The triangle with vertices $a, b$ and $b^{\prime}$ misses $\mathcal{H}$ because it lies in $A$. By symmetry, the $R$-images of this pentagon and triangle also miss $\mathcal{H}$. The $\frac{1}{3}$-tube, two pentagons and two triangles fit together to give a homotopy between $\mu_{a, A, H}$ and $\mu_{a, A, q, H}$.

We will also need a version of this where the basepoint is an ordinary point of $\mathbb{B}^{13}-\mathcal{H}$ rather than a Leech cusp. The picture and proof differ from lemma 3.5 only by replacing all of $\rho, A, a, b$ and $b^{\prime}$ by a single point $b$.

Lemma 3.6 (Homotopies between meridians based at a point of $\mathbb{B}^{13}$ ). Suppose $b \in \mathbb{B}^{13}-\mathcal{H}$. Let $H$ be a mirror, $p$ be the point of $H$ closest to $b$, and $q$ be another point of $H$. Suppose that the totally real triangle $\triangle b p q$ is disjoint from $\mathcal{H}$ except that $\overline{p q}$ lies in $H$ and that $q$ might lie in additional mirrors. Then $\mu_{b, H} \simeq \mu_{b, q, H}$ rel endpoints in $\mathbb{B}^{13}-\mathcal{H}$, and $M_{b, H}=M_{b, q, H}$ in $G_{b}$.

\section{Finitely many Generators Based "AT" a CUSP}

In this section we begin the proof of our main result, theorem 1.2. Our starting point is theorem 1.4 from $[\mathrm{AB}$, which states that the set of all Leech meridians generates $G_{a}=\pi_{1}^{\text {orb }}\left(\left(\mathbb{B}^{13}-\mathcal{H}\right) / P \Gamma, a\right)$, where $a$ is a basepoint chosen near a Leech cusp $\rho$. Recall from section 2.8 that the Leech roots are the roots $r$ satisfying $\langle\rho \mid r\rangle=\theta$ and Leech meridians are the meridians $M_{a, A, s}$ with $s$ varying over the Leech roots, where $A$ is a closed horoball centered at $\rho$, disjoint from $\mathcal{H}$ and containing $a$. This is an infinite generating set, which we improve in theorem 4.1 by exhibiting an explicit finite set of Leech meridians that generates $G_{a}$. The exact nature of this generating set is not so important, but it must be explicit and finite so that we will be able to prove theorem 5.1 in the next section. 
Theorem 4.1 (130 generators based "at" a cusp). Suppose $\rho=(3 \omega-1 ;-1, \ldots,-1)$ is the Leech cusp from section 2.8. $A$ is a closed horoball centered at $\rho$ and disjoint from $\mathcal{H}$, and $a$ is an element of $A$. Write $S$ for the set of 130 Leech roots

$$
p_{i}, \quad p_{i}-\rho, \quad \bar{\omega} p_{i}-l_{j}, \quad \bar{\omega} p_{i}-l_{j}-\rho
$$

with $i, j=1, \ldots, 13$ and $p_{i}$ and $l_{j}$ incident in the last two cases. Then $G_{a}$ is generated by the Leech meridians $M_{a, A, s}$, where $s$ varies over $S$.

In this section we will write $G$ for the subgroup of $G_{a}$ generated by these particular Leech meridians, and our goal is to show that $G$ is all of $G_{a}$. The strategy is to show that $G$ contains some isometries of $\mathbb{B}^{13}$ that fix $\rho$ and act transitively on the Leech roots. It follows from this that every Leech meridian is $G$-conjugate into $G$, hence lies in $G$. Since the set of all Leech meridians generates $G_{a}$, it follows that $G$ is all of $G_{a}$, as desired.

But $G_{a}$ is an orbifold fundamental group, not a group of isometries of $\mathbb{B}^{13}$, so we must say what we mean by $G_{a}$ "containing" some isometries of $\mathbb{B}^{13}$. We will explain this and then develop the details of the strategy just outlined. The idea is that the subgroup $P \Gamma_{A}$ of $P \Gamma \subseteq \operatorname{Isom}\left(\mathbb{B}^{13}\right)$ preserving $A$ can be thought of as a subgroup of $G_{a}$. To see this, consider the obvious homomorphism

$$
P \Gamma_{A}=\pi_{1}^{\text {orb }}\left(A / P \Gamma_{A}, a\right) \rightarrow \pi_{1}^{\text {orb }}\left(\left(\mathbb{B}^{13}-\mathcal{H}\right) / P \Gamma, a\right)=G_{a} .
$$

The first equality holds because $A$ is simply connected, and the map exists because $A$ misses $\mathcal{H}$. This map $P \Gamma_{A} \rightarrow G_{a}$ is an embedding, because following it by the natural map $G_{a} \rightarrow P \Gamma$ (see section 2.9) gives the identity on $P \Gamma_{A}$. In this manner, we may regard $P \Gamma_{A}$ as a subgroup of $G_{a}$. Concretely, if $g \in P \Gamma_{A}$, then the corresponding element of $G_{a}$ is $(\gamma, g)$ where $\gamma$ is any path in $A$ from $a$ to $g(a)$. The choice of $\gamma$ is unimportant since $A$ is simply connected.

The elements of $P \Gamma_{A}$ we will need are the following "translations" $T_{\lambda, z}$. The language comes from their analogy with the isometries of real hyperbolic space which look like Euclidean translations in the upper half space model. Given $\lambda \in \Lambda$ and $z \in \operatorname{Im} \mathbb{C}$ such that $z-\lambda^{2} / 2 \in \theta \mathcal{E}$, the definition is

$$
\begin{aligned}
(l ; 0,0) & \mapsto\left(l ; 0, \bar{\theta}^{-1}\langle l \mid \lambda\rangle\right) \\
T_{\lambda, z}:(0 ; 1,0) & \mapsto\left(\lambda ; 1, \theta^{-1}\left(z-\lambda^{2} / 2\right)\right) \\
(0 ; 0,1) & \mapsto(0 ; 0,1)
\end{aligned}
$$

One can see that the translations form a Heisenberg group by checking the identities:

$$
\begin{aligned}
T_{\lambda, z} T_{\lambda^{\prime}, z^{\prime}} & =T_{\lambda+\lambda^{\prime}, z+z^{\prime}+\operatorname{Im}\left\langle\lambda \mid \lambda^{\prime}\right\rangle} \\
\left(T_{\lambda, z}\right)^{-1} & =T_{-\lambda,-z} \\
T_{\lambda, z} T_{\lambda^{\prime}, z^{\prime}} T_{\lambda, z}^{-1} T_{\lambda^{\prime}, z^{\prime}}^{-1} & =T_{0,2 \operatorname{Im}\left\langle\lambda \mid \lambda^{\prime}\right\rangle}
\end{aligned}
$$

This group preserves $A$ and $\rho$, and acts simply transitively on the Leech roots. The center of this group consists of $T_{0, z}$ given by $T_{0, z}(v ; 1, \alpha)=\left(v ; 1, \alpha+\theta^{-1} z\right)$ where $z \in \theta \mathbb{Z}$. The next lemma, which is the crucial trick in this section, obtains some elements of $P \Gamma_{A}$ as products of meridians. Then we combine these in lemma 4.3 to obtain translations.

Lemma 4.2. Suppose $\rho \in L$ is a Leech cusp, $A$ is a closed horoball centered at $\rho$ and disjoint from $\mathcal{H}$, and $a \in A$. Suppose $s$ is a Leech root, and write $s^{\prime}$ for 


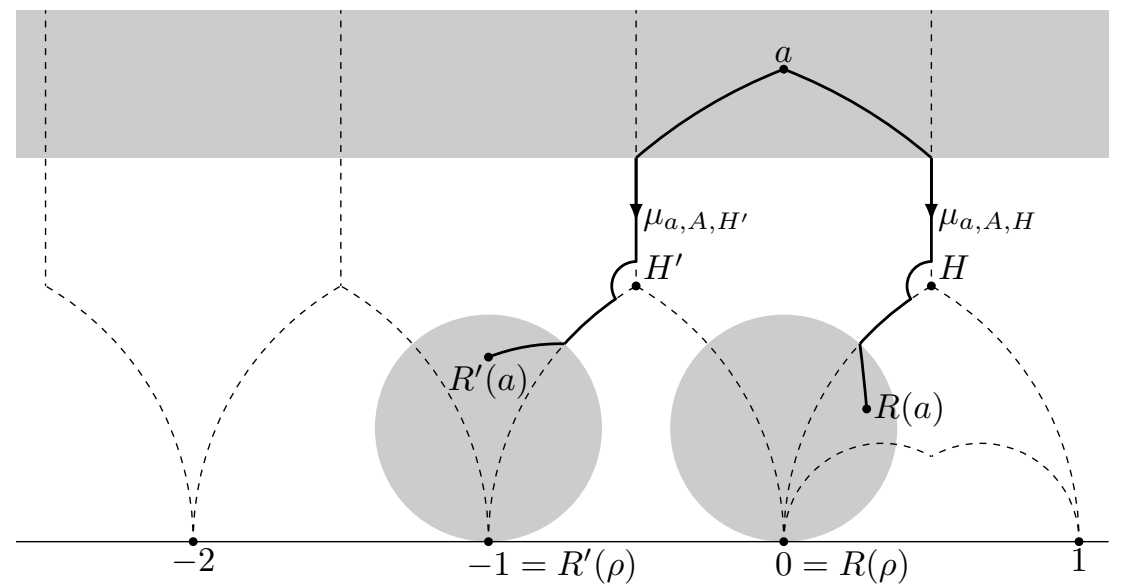

Figure 4.1. The Leech meridians appearing in (4.6). The cusp $\rho$ is at infinity and the hyperplanes $H$ and $H^{\prime}$ appear as the points where they meet the pictured $\mathbb{B}^{1}$. The top gray region is the horoball $A$, and the regions below it are its images under $R$ and $R^{\prime}$.

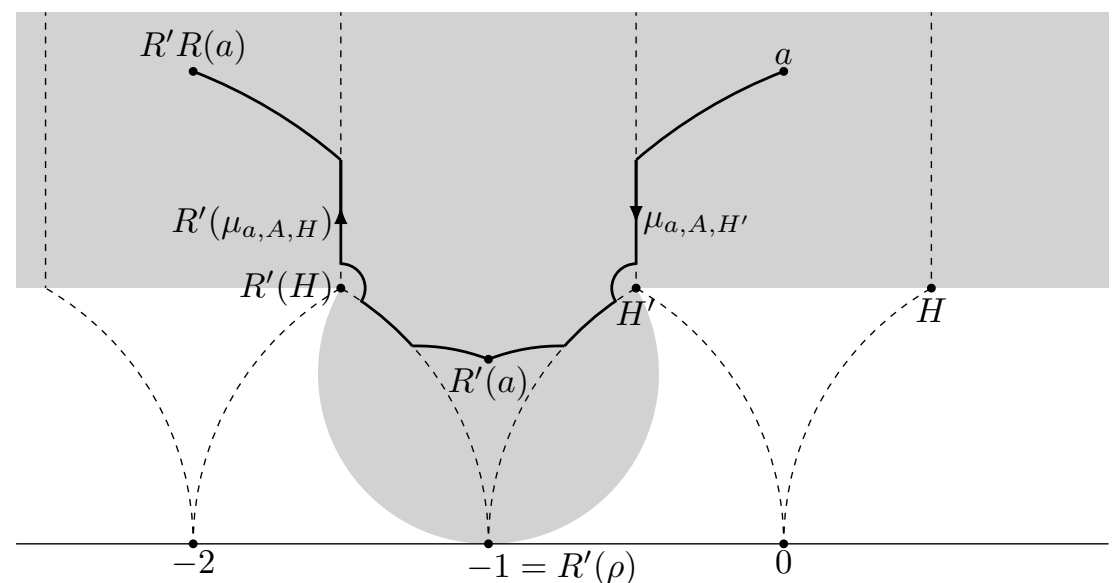

Figure 4.2. The proof of lemma 4.2. The gray region is the union of the open height 1 horoballs around $\rho$ and $R(\rho)$. Since these horoballs miss $\mathcal{H}$, the path from $a$ to $R^{\prime} R(a)$ is homotopic into $A$.

the Leech root $s-\rho$. Write $H$ and $H^{\prime}$ for their mirrors and $R$ and $R^{\prime}$ for their $\omega$-reflections. Then

$$
M_{a, A, H^{\prime}} \cdot M_{a, A, H}=R^{\prime} \cdot R
$$

Here the product of meridians on the left is evaluated in the orbifold fundamental group $G_{a}$, and the product of reflections on the right is evaluated in $P \Gamma$, with result in $P \Gamma_{A}$. When stating equality we are regarding $P \Gamma_{A}$ as a subgroup of $G_{a}$ via (4.1). 
Proof. We use the Leech model of $L$, with $\rho=(0 ; 0,1)$ as usual. By the transitivity of translations on Leech roots, we may suppose without loss of generality that $s=(0 ; 1,-\omega)$, so $s^{\prime}=(0 ; 1, \bar{\omega})$. Consider the subspace $V=\{(0 ; x, y)\}$ of $L \otimes \mathbb{C}$. We plot the point in $P(V) \cong P^{1}$ represented by $(0 ; x, y)$ as $y / x \in \mathbb{C} \cup\{\infty\}$. Using this, we have drawn the situation in figure 4.1. In particular, $\rho$ corresponds to the point at infinity and $\mathbb{B}^{1}=\mathbb{B}(V)$ to the upper half plane. The orthogonal complements in $V$ of $s$ and $s^{\prime}$ are spanned by $(0 ; 1,-\bar{\omega})$ and $(0 ; 1, \omega)$ respectively. So $H=s^{\perp}$ and $H^{\prime}=s^{\perp}$ correspond to $-\bar{\omega}$ and $\omega$ in the upper half plane. The triflections $R^{\prime}$ and $R$ act by counterclockwise hyperbolic rotations by $2 \pi / 3$ around these points. It is easy to see that $R(\infty)=0, R^{\prime}(0)=\infty$ and $R^{\prime}(\infty)=-1$. In particular, $R^{\prime} R \in P \Gamma_{A}$, which is part of the lemma. The closed horoball $A$ and its images under $R$ and $R^{\prime}$ also appear in figure 4.1. To draw the figure we have assumed that $a$, a priori any point of $A$, actually lies in $\mathbb{B}^{1}$ and has real part 0 . This is harmless, by remark 3.3

By definition (section 2.9), the left side of (4.6) is equal to

$$
\left(\mu_{a, A, H^{\prime}}, R^{\prime}\right) \cdot\left(\mu_{a, A, H}, R\right)=\left(\mu_{a, A, H^{\prime}} \text { followed by } R^{\prime} \circ \mu_{a, A, H}, R^{\prime} R\right)
$$

The path part of the right side appears in figure 4.2, This figure is the same as figure 4.1 except as follows. First, we have drawn $R^{\prime} \circ \mu_{a, A, H}$ instead of $\mu_{a, A, H}$. Second, we have drawn the height 1 open horoballs around $\rho=\infty$ and $R^{\prime}(\rho)=-1$, instead of $A$ and its translates. It is easy to see that $\mu_{a, A, H^{\prime}}$ followed by $R^{\prime} \circ \mu_{a, A, H}$ lies in the union of these two horoballs. (One must check that $s^{\prime}$ and $R^{\prime}(s)$ are first-shell roots with respect to $R^{\prime}(\rho)$, which is almost obvious.) The union of these horoballs is contractible and misses $\mathcal{H}$. So our path from a to $R^{\prime} R(a)$ may be homotoped (rel endpoints and missing $\mathcal{H}$ ) to a path in $A$. We have proven that the left side of (4.6) is equal to

$$
\text { (some path in } A \text { from } a \text { to } R^{\prime} R(a), R^{\prime} R \text { ) }
$$

in the orbifold fundamental group $G_{a}$. This is exactly what the equality (4.6) asserts, so the proof is complete.

Remark. Figures 4.1 and 4.2 bring to mind the classical action of $\mathrm{SL}_{2} \mathbb{Z}$ on the upper half plane. This is because the projective isometry group of $\left(\begin{array}{ll}0 & \bar{\theta} \\ \theta & 0\end{array}\right)$ happens to be $\mathrm{PSL}_{2} \mathbb{Z}$. The dashed lines indicate some fundamental domains for the index 2 subgroup generated by triflections.

Lemma 4.3. Suppose $\rho, A$ and $a$ are as in lemma 4.2, and $S_{0}$ is a set of Leech roots whose differences span $\rho^{\perp} /\langle\rho\rangle$. Then the subgroup of $P \Gamma_{A}$ generated by the products $R^{\prime} R$ of lemma 4.2. with $s$ varying over $S_{0}$, contains all the translations (4.2). In particular, it acts transitively on the Leech roots.

Proof. At heart this is lemma 8 of [A3]. But the statement there, and conventions and notation, are different enough that we indicate the proof. Write $G$ for the specified subgroup of $P \Gamma_{A}$.

We begin by taking $r$ to be the Leech root $(0 ; 1,-\omega)$ and setting $r^{\prime}=r-\rho=$ $(0 ; 1, \bar{\omega})$. One can check that on the span of these vectors, $R_{r}^{\prime} R_{r}$ acts by $-\omega T_{0,-2 \theta}$ (Here, as in lemma 4.2, using a prime means replacing a Leech root by a translate of itself and replacing the complex reflection in the corresponding way). Obviously $R_{r}^{\prime} R_{r}$ acts trivially on $\Lambda$. We re-express this by saying that $R_{r}^{\prime} R_{r}$ acts on $L$ by $-\omega Q T_{0,-2 \theta}$, where $Q$ scales $\Lambda \subseteq L$ by $-\bar{\omega}$ and fixes $r$ and $r^{\prime}$. One can check that $Q T_{\lambda, z} Q^{-1}=T_{-\bar{\omega} \lambda, z}$ for every translation $T_{\lambda, z}$. 
Now suppose $s \in S_{0}$. As a Leech root, we have $s=T_{\sigma, y}(r)$ for some translation $T_{\sigma, y}$, and we also have $s^{\prime}=T_{\sigma, y}\left(r^{\prime}\right)$ because $s^{\prime}=s-\rho$ and $r^{\prime}=r-\rho$. Therefore

$$
R_{s}^{\prime} R_{s}=T_{\sigma, y}\left(R_{r}^{\prime} R_{r}\right) T_{\sigma, y}^{-1}=T_{\sigma, y} \cdot-\bar{\omega} Q T_{0,-2 \theta} \cdot T_{\sigma, y}^{-1}
$$

Choosing another element $t$ of $S_{0}$ and writing it as $T_{\tau, z}(r)$, we get a similar description of $R_{t}^{\prime} R_{t}$. So

$$
\begin{aligned}
\left(R_{s}^{\prime} R_{s}\right)\left(R_{t}^{\prime} R_{t}\right)^{-1} & =T_{\sigma, y} \cdot\left(-\omega Q T_{0,-2 \theta}\right) \cdot T_{\sigma, y}^{-1} \cdot T_{\tau, z} \cdot\left(-\bar{\omega} T_{0,2 \theta} Q^{-1}\right) \cdot T_{\tau, z}^{-1} \\
& =T_{\sigma, y} Q\left(T_{-\sigma,-y} T_{\tau, z}\right) Q^{-1} T_{\tau, z}^{-1} \\
& =T_{\sigma, y} T_{\bar{\omega} \sigma,-y} T_{-\bar{\omega} \tau, z} T_{\tau, z}^{-1} \\
& =T_{\sigma+\bar{\omega} \sigma-\bar{\omega} \tau-\tau, ?} \\
& =T_{-\omega(\sigma-\tau), ?}
\end{aligned}
$$

where the value of "?" could be worked out but is unimportant for us.

We have shown that for each pair $s, t \in S_{0}, G$ contains a translation $T_{-\omega(\sigma-\tau), ?}$. So it also contains a translation $\left(R_{s}^{\prime} R_{s}\right) T_{-\omega(\sigma-\tau), ?}\left(R_{s}^{\prime} R_{s}\right)^{-1}=T_{\sigma-\tau, ?}$. We have shown that for all $s, t \in S_{0}, G$ contains a translation $T_{\sigma-\tau, ?}$ and a translation $T_{-\omega(\sigma-\tau), ?}$. It follows from (4.3) that for every $\lambda$ in the $\mathcal{E}$-span of the differences $\sigma-\tau, G$ contains a translation $T_{\lambda, ?}$.

By hypothesis, the differences $s-t$, with $s, t \in S_{0}$, projected into $\rho^{\perp} /\langle\rho\rangle$, span $\rho^{\perp} /\langle\rho\rangle$ over $\mathcal{E}$. Another way to say this is the $\mathcal{E}$-span of the corresponding differences $\sigma-\tau$ is all of $\Lambda$. So we have shown that for every $\lambda \in \Lambda, G$ contains a translation $T_{\lambda, ?}$. Using these in the commutator formula (4.5), and using $\Lambda=\theta \Lambda^{*}$, shows that $G$ also contains $T_{0, z}$ for every $z \in \operatorname{Im} \mathcal{E}$. It follows that $G$ contains all translations.

Lemma 4.4. Suppose $\rho, A, a$ and $S_{0}$ are as in lemma 4.3, and define

$$
S=\left\{s, s-\rho \mid s \in S_{0}\right\} .
$$

Then $G_{a}$ is generated by the Leech meridians $M_{a, A, s}$, where $s$ varies over $S$.

Proof. Write $G$ for the subgroup of $G_{a}$ generated by these Leech meridians. Also, fix some $s \in S$. For any other Leech root $t$, there is a translation $g \in P \Gamma_{A}$ that sends $s$ to $t$. We choose a path $\gamma$ in $A$ from $a$ to $g(a)$, and identify $g$ with the element $(\gamma, g)$ of the orbifold fundamental group $G_{a}$, via (4.1). By the previous lemma we know that $(\gamma, g)$ lies in $G$. By drawing a picture, it is easy to see that

$$
(\gamma, g) \cdot\left(\mu_{a, A, s}, R_{s}\right) \cdot(\gamma, g)^{-1}=\left(\mu_{a, A, t}, R_{t}\right)
$$

in $G_{a}$, so $G$ also contains the Leech meridian associated to $t$. Since $t$ was an arbitrary Leech root, $G$ contains all the Leech meridians. These generate $G_{a}$ by theorem 1.4 of $\mathrm{AB}$, completing the proof that $G=G_{a}$.

Proof of theorem 4.1. This follows from the previous lemma with $S_{0}$ equal to the set of Leech roots $p_{i}$ and $\bar{\omega} p_{i}-l_{j}$, where $i, j=1, \ldots, 13$ and $p_{i}$ and $l_{j}$ are incident. To apply the lemma, we must show that the image of $K$ in $\rho^{\perp} /\langle\rho\rangle$ is equal to $\rho^{\perp} /\langle\rho\rangle$, where $K$ is the $\mathcal{E}$-span of $\left\{s-t: s, t \in S_{0}\right\}$. We show that in fact $K=\rho^{\perp}$.

Note that $\rho$ has the same inner product with all elements of $S_{0}$. So $K \subseteq \rho^{\perp}$. For the converse, let $K_{1}$ be the $\mathcal{E}$-span of $\left\{\left(p_{i}-p_{1}\right),\left(l_{i}-l_{1}\right): i=1, \cdots, 13\right\}$. Observe that $K$ contains $\left(p_{1}-p_{i}\right)$. Given a line root $l_{i}$, let $p_{j}$ be the point root incident on 
$l_{i}$ and $l_{1}$. Writing $\left(l_{i}-l_{1}\right)=\left(\bar{\omega} p_{j}-l_{1}\right)-\left(\bar{\omega} p_{j}-l_{i}\right)$ we find that $K$ contains $\left(l_{i}-l_{1}\right)$ too. We have now shown that $K_{1} \subseteq K$.

Now suppose $v \in \rho^{\perp}$; we must show that it lies in $K$. By adding a multiple of $\left(\bar{\omega} p_{1}-l_{1}\right)-p_{1} \in K$, which has inner product $\theta$ with $p_{\infty}$, we may suppose without loss of generality that $v \perp p_{\infty}$. Being orthogonal to $\rho$ and $p_{\infty}$, the vector $v$ is also orthogonal to $l_{\infty}$. Since $L$ is spanned by the point and line roots, we may write $v=y+a p_{1}+b l_{1}$ for some $y \in K_{1}$ and $a, b \in \mathcal{E}$. Taking inner product with $p_{\infty}$ and $l_{\infty}$ and using the fact that $p_{\infty}, l_{\infty} \in K_{1}^{\perp}$ shows $a=b=0$. So $v=y \in K_{1} \subseteq K$.

\section{TWENTY-SIX GENERATORS BASED "AT" A CUSP}

The goal of this section is to prove the theorem 5.1 below. We recall that in the $P^{2} \mathbb{F}_{3}$ model of $L$ from section 2.4 , the point-roots are the 13 vectors of the form $\left(0 ; \theta, 0^{12}\right)$ and the line-roots are the 13 vectors of the form $\left(1 ; 1^{4}, 0^{9}\right)$. We also recall from section 2.8 that the null vector $\rho=(3 \omega-1 ;-1, \ldots,-1)$ represents a Leech cusp, that $A$ indicates a closed horoball centered at $\rho$, small enough to miss $\mathcal{H}$, and that $a \in A$ indicates the basepoint. Finally, recall that if $s$ is a point- or line-root, then we will call the meridian $M_{a, A, s} \in G_{a}$ the corresponding point- or line-meridian (based at $a$ ).

Theorem 5.1 (26 generators based "at" a cusp). The 26 point- and line-meridians $M_{a, A, s}$, based at a, generate the orbifold fundamental group $G_{a}$.

To prove it we will show that the subgroup $G$ of $G_{a}$ generated by the point- and line-meridians contains the 130 Leech meridians from theorem 4.1. That theorem says that the 130 meridians generate $G_{a}$, so theorem 5.1 follows. We remark that the point-roots are Leech roots with respect to $\rho$, so their corresponding meridians are Leech meridians. The line-roots are 2 nd-shell roots rather than Leech roots.

Our recipe for getting additional Leech meridians from the point- and linemeridians is the following. Suppose $x$ and $y$ are Leech roots with inner product $\pm \theta$, or a point-root and an incident line-root, in which case they also have inner product $\pm \theta$. The span of two roots with inner product $\pm \theta$ is a well-known Eisenstein lattice, called $D_{4}^{\mathcal{E}}$ because its underlying $\mathbb{Z}$-lattice is a copy of the usual $D_{4}$ root lattice, scaled to have minimal norm 3 . Among the 24 roots in $\langle x, y\rangle$, it turns out that there is exactly one Leech root that is not a scalar multiple of $x$ or $y$. Call it $z$. We will show that the subgroup of $G_{a}$ generated by $M_{a, A, x}$ and $M_{a, A, y}$ also contains $M_{a, A, z}$. "New" Leech meridians obtained this way can be combined with old ones using the same method, to obtain even more Leech meridians. Repeating the process many times enables us to prove that $G$ contains the 130 Leech meridians of theorem 4.1 .

This idea suggested itself because the braid group associated to the complex reflection group Aut $D_{4}^{\mathcal{E}}$ is known to be isomorphic to the usual 3-strand braid group $B_{3}$, with the conjugacy class of our meridians corresponding to the conjugacy class of the standard generators of $B_{3}$. It was natural to hope that the meridians $M_{a, A, x}, M_{a, A, y}$ and $M_{a, A, z}$ all lie in a single copy of $B_{3}$, with the first two of them serving as generators. So our first result is that the braid group of $D_{4}^{\mathcal{E}}$ is generated by two specific meridians, for either of two specific basepoints. It seems silly to have to prove this, since the braid groups of finite complex reflections groups are well understood $\mathrm{Ban} \mathrm{Be} \mathrm{Mi}$. But we do not know of any quotable result stating generation by particular loops with particular basepoints. 
Lemma 5.2 (Generators for the braid group of $D_{4}^{\mathcal{\varepsilon}}$ ). Consider the lattice $D_{4}^{\mathcal{E}}$ in $\mathbb{C}^{2}$, namely the $\mathcal{E}$-span of $\alpha=(\theta, 0)$ and $\beta=(1, \sqrt{2})$ under the usual inner product. Let $\mathcal{H}$ be the union of the mirrors orthogonal to the 24 roots of $D_{4}^{\mathcal{E}}$, and let $c$ be any scalar multiple of $(-1,-\bar{\omega} \sqrt{2})$ or $\frac{\omega}{2}(\theta+1,(\theta-2) \sqrt{2})$. Then $J_{c}=\pi_{1}^{\text {orb }}\left(\left(\mathbb{C}^{2}-\mathcal{H}\right) /\left\langle R_{\alpha}, R_{\beta}\right\rangle, c\right)$ is generated by $M_{c, \alpha}$ and $M_{c, \beta}$.

Remark. The $\sqrt{2}$ 's can be avoided by using three coordinates with the last two being equal: $\alpha=(\theta, 0,0)$ and $\beta=(1,1,1)$.

Proof. We begin by remarking that $\alpha^{2}=\beta^{2}=3$ and $\langle\alpha \mid \beta\rangle=\theta$. We write $\gamma=$ $(1, \bar{\omega} \sqrt{2})$ and $\delta=(1, \omega \sqrt{2})$ for the remaining roots of $D_{4}^{\mathcal{E}}$, up to scale. We will begin the proof by establishing the lemma for a different basepoint $c_{0}=(-2, \sqrt{2}+\sqrt{6})$. This basepoint is probably the most natural: when one plots the situation in $\mathbb{C} P^{1}$, the four mirrors form the vertices of a regular tetrahedron and $c_{0}$ corresponds to the midpoint of the edge between $\alpha^{\perp}$ and $\beta^{\perp}$. We will suppress the subscript 0 until we need to refer to the other $c$ 's.

One can check that $\langle c \mid \alpha\rangle$ and $\langle c \mid \beta\rangle$ are equal in absolute value, and smaller in absolute value than $\langle c \mid \gamma\rangle$ and $\langle c \mid \delta\rangle$. Therefore $\alpha^{\perp}$ and $\beta^{\perp}$ are the mirrors nearest $c$. Write $J$ for the subgroup of $J_{c}$ generated by $M_{c, \alpha}=\left(\mu_{c, \alpha}, R_{\alpha}\right)$ and $M_{c, \beta}=\left(\mu_{c, \beta}, R_{\beta}\right)$. We are claiming that $J$ is all of $J_{c}$. Theorem 1.2 of $\mathrm{AB}$ gives sufficient conditions for this. First, $J$ should surject to $\left\langle R_{\alpha}, R_{\beta}\right\rangle$, which is obvious. Second, some triflection in $\alpha$ or $\beta$ should move $c$ closer to $c$ 's projection to $\gamma^{\perp}$, and some triflection should do the same with $\delta$ in place of $\gamma$. Direct calculation reveals that $R_{\alpha}$ does this for $\gamma^{\perp}$ and $R_{\alpha}^{-1}$ does it for $\delta^{\perp}$. This finishes the proof of the lemma for the basepoint $c_{0}$.

A different method is required for the other basepoints. First we suppose the basepoint $c$ is a scalar multiple of $(-1,-\bar{\omega} \sqrt{2})$. It does not matter which multiple, because scaling permutes the possibilities (and transforms their meridians accordingly). We choose $c$ to be the particular multiple $c_{1}=(\theta \bar{\omega}-\omega \sqrt{3})(-1,-\bar{\omega} \sqrt{2})$. The advantage of this scaling is that $\left\langle c_{0} \mid c_{1}\right\rangle$ is positive, so the angle between $c_{0}$ and $c_{1}$ is as small as possible. We regard this as saying that $c_{1}$ is "as close as possible" to $c_{0}$, which is desirable because we will study the identification of $J_{c_{0}}$ with $J_{c_{1}}$ got by moving $c_{0}$ to $c_{1}$ along $\overline{c_{0} c_{1}}$. Namely, will show that $M_{c_{0}, \alpha} \in J_{c_{0}}$ corresponds to $M_{c_{1}, \alpha} \in J_{c_{1}}$, and similarly with $\beta$ in place of $\alpha$. From this correspondence and the previous paragraph it follows immediately that $M_{c_{1}, \alpha}$ and $M_{c_{1}, \beta}$ generate $J_{c_{1}}$.

We will write $p_{i}$ for the projection of $c_{i}$ to $\alpha^{\perp}$. One can check that the convex hull $T$ of $c_{0}, p_{0}$ and $c_{1}$ meets $\mathcal{H}$ only at $p_{0}$. (Let $\langle T \mid \alpha\rangle$ be the triangle in $\mathbb{C}$ with vertices $\left\langle c_{0} \mid \alpha\right\rangle,\left\langle p_{0} \mid \alpha\right\rangle,\left\langle p_{1} \mid \alpha\right\rangle$. One checks that the triangle $\langle T \mid \alpha\rangle$ touches the origin only at its vertex $\left\langle p_{0} \mid \alpha\right\rangle$. Similarly one checks that $\beta^{\perp}, \gamma^{\perp}, \delta^{\perp}$ do not intersect $T$ by checking that the triangles $\langle T \mid \beta\rangle,\langle T \mid \gamma\rangle,\langle T \mid \delta\rangle$ do not contain the origin.) The obvious variant of Lemma 3.2 for Euclidean space implies that moving the basepoint from $c_{0}$ to $c_{1}$ along $\overline{c_{0} c_{1}}$ identifies $M_{c_{0}, \alpha} \in J_{c_{0}}$ with $M_{c_{1}, p_{0}, \alpha} \in J_{c_{1}}$. Similarly, one checks that the convex hull of $p_{0}, c_{1}$ and $p_{1}$ misses $\mathcal{H}$ except that $\overline{p_{0} p_{1}} \subseteq \alpha^{\perp}$. From this, lemma 3.6 concludes that $M_{c_{1}, p_{0}, \alpha}$ is the same element of $J_{c_{1}}$ as $M_{c_{1}, \alpha}$. We have proven the correspondence between $M_{c_{0}, \alpha}$ and $M_{c_{1}, \alpha}$. The same method applies with $\beta$ in place of $\alpha$. This completes the proof for the basepoint $c_{1}$. 
Exactly the same analysis applies to the second possibility for $c$. The scalar multiple of $\frac{\omega}{2}(\theta+1,(\theta-2) \sqrt{2})$ that we used was

$$
c_{2}=-(3+2 \sqrt{3}+\theta \sqrt{3})(\theta+1,(\theta-2) \sqrt{2}),
$$

for the same reason as before.

As explained above, our method starts with two Leech meridians, corresponding to Leech roots with inner product $\pm \theta$, and shows that the group they generate contains a third Leech meridian. So naturally we will need to understand such pairs of Leech roots. At the same time we will classify the pairs of Leech roots with inner product $-\frac{3}{2} \pm \frac{\theta}{2}$. We do not care about these pairs themselves. But they turn out to be key for the other half of our method: using a point-meridian and a line-meridian to generate another Leech meridian. The precise statements of our constructions of "new" Leech meridians are lemmas 5.4(3) and 5.6.

Lemma 5.3. Under the $\Gamma$-stabilizer $\Gamma_{\rho}$ of $\rho$, there are four orbits of ordered pairs of Leech roots with inner product of absolute value $\sqrt{3}$. In the Leech model of $L$, orbit representatives are the pairs $\left(s, s^{\prime}\right)$ where

$$
\begin{array}{ll}
s=(0 ; 1,-\omega) \text { and } s^{\prime}=\left(\lambda_{6} ; 1, \omega\right), & \text { with }\left\langle s \mid s^{\prime}\right\rangle=\theta \\
s=(0 ; 1,-\omega) \text { and } s^{\prime}=\left(\lambda_{9} ; 1, \theta\right), & \text { with }\left\langle s \mid s^{\prime}\right\rangle=-\frac{3}{2}+\frac{\theta}{2}=\bar{\omega} \bar{\theta}
\end{array}
$$

and the pairs got from these by exchanging $s, s^{\prime}$. Here $\lambda_{6}$ and $\lambda_{9}$ are any fixed vectors of norms 6 and 9 in $\Lambda$.

Proof. Suppose $\left(s, s^{\prime}\right)$ is such an ordered pair. In the Leech model, the Leech roots are parameterized by (2.8). So $s$ has the form $\left(\sigma ; 1, \theta\left(\frac{\sigma^{2}-3}{6}+\nu\right)\right)$ where $\sigma$ lies in the Leech lattice and $\nu$ is purely imaginary and chosen so that the last coordinate lies in $\mathcal{E}$. And similarly for $s^{\prime}$. By the transitivity of $\Gamma_{\rho}$ on Leech roots, we may take $\sigma=0$ and $\nu=\frac{1}{2 \theta}$, yielding $s=\left(0 ; 1, \theta\left(-\frac{1}{2}+\frac{1}{2 \theta}\right)\right)=(0 ; 1,-\omega)$. The inner product $\left\langle s \mid s^{\prime}\right\rangle$ is best understood using (2.7), which in this case reduces to

$$
\left\langle s \mid s^{\prime}\right\rangle=\frac{6-\sigma^{\prime 2}}{2}+3\left(\nu^{\prime}-1 / 2 \theta\right) .
$$

The first term is real and the second is imaginary. Now we consider the elements of $\mathcal{E}$ of absolute value $\sqrt{3}$. First, $\frac{3}{2} \pm \frac{\theta}{2}$ cannot occur, because $\sigma^{\prime}$ would have to have norm 3 in order for $\left\langle s \mid s^{\prime}\right\rangle$ to have real part $\frac{3}{2}$. This is impossible since the Leech lattice has minimal norm 6 . By the same reasoning, $\pm \theta$ can occur only when $\sigma^{\prime}$ has norm 6 , and $-\frac{3}{2} \pm \frac{\theta}{2}$ can occur only when $\sigma^{\prime}$ has norm 9 . Since Aut $\Lambda=6 \cdot S u z$ acts transitively on the norm 6 and norm 9 elements of $\Lambda$, we may suppose without loss of generality that $\sigma^{\prime}=\lambda_{6}$ or $\lambda_{9}$. Then $\nu^{\prime}$ is determined by the imaginary part of $\left\langle s \mid s^{\prime}\right\rangle$. When $\left\langle s \mid s^{\prime}\right\rangle=\theta$ we get $3\left(\nu^{\prime}-\frac{1}{2 \theta}\right)=\theta$, so $\nu^{\prime}=-\frac{1}{2 \theta}$ and $s^{\prime}$ is as displayed. Similarly, when $\left\langle s \mid s^{\prime}\right\rangle=-\frac{3}{2}+\frac{\theta}{2}$ one gets $\nu^{\prime}=0$.

The proofs of the next two lemmas depend on how certain totally real triangles in $\mathbb{B}^{13}$ meet $\mathcal{H}$. Because the calculations are long, and similar computations will be needed later, we moved the verifications to appendix $\mathrm{A}$ Lemma 5.4 relies on lemma A.3 and lemma 5.5 relies on lemma A.4.

Lemma 5.4. Suppose $x, y$ are Leech roots with $\langle x \mid y\rangle=\theta, p$ is the projection of $\rho$ to $x^{\perp}$, and $q$ is the projection of $\rho$ to the $x^{\perp} \cap y^{\perp}$. Then 
(1) Of the four mirrors of the reflection group $\left\langle R_{x}, R_{y}\right\rangle$, three are Leech mirrors (corresponding to the Leech roots $x, y$ and $z=-\bar{\omega} x-\omega y$ ) and one is a second-shell mirror.

(2) These four mirrors are the only ones containing q.

(3) The subgroup of $G_{a}$ generated by the Leech meridians $M_{a, A, x}$ and $M_{a, A, y}$ also contains the Leech meridian $M_{a, A, z}$.

Remark. An example of the situation described in this lemma is given by $x=$ $-R_{p_{2}}(l)$ and $y=p$, where $l$ is a line-root and $p, p_{2}$ are the two point-roots incident on $l$. We formulated our notion of "meridian" to include cases where the basepoint is not in general position. The situation in this lemma is such a case, because $q$ is the projection of $\rho$ to the second-shell mirror in (11), and is only one of 4 mirrors passing through $q$. In fact, one can prove that this is the only sort of non-genericity that occurs for our lattice $L$.

Proof. By lemma 5.3 we may suppose without loss of generality that $x=(0 ; 1,-\omega)$ and $y=\left(\lambda_{6} ; 1, \omega\right)$. And we suppose without loss of generality that our basepoint $a$ lies in $\overline{\rho q} \cap A$ (see remark 3.3).

(11) Using $x^{2}=y^{2}=3$ and $\langle x \mid y\rangle=\theta$, one checks that $z$ and $\omega x-\bar{\omega} y$ are roots. Then, using $\langle\rho \mid x\rangle=\langle\rho \mid y\rangle=\theta$, one checks that $\langle\rho \mid z\rangle=\theta$, so that $z$ is also a Leech root, and that $\langle\rho \mid \omega x-\bar{\omega} y\rangle=3$, so that $(\omega x-\bar{\omega} y)^{\perp}$ is a second-shell mirror.

(2) Lemma A.3 identifies all the mirrors that meet the totally real triangle $\triangle \rho p q$. The only ones that do are the four from (1). So they are the only ones that can contain $q$.

Before proving (3) we establish two preparatory results. First, there is an isometry of $L$ that fixes $\rho$ and permutes $x, y$ and $z$ cyclically. To see this, one checks that $\langle y \mid z\rangle=\theta$ and applies the transitivity in lemma 5.3 to conclude that some $g \in \Gamma_{\rho}$ sends $(x, y)$ to $(y, z)$. Now, $g$ must permute the three Leech roots in $\langle x, y\rangle=\langle y, z\rangle$. Since $g$ sends $x$ to $y$ and $y$ to $z$, it must also send $z$ to $x$.

Second, we claim that $\mu_{a, A, x}$ is homotopic to $\mu_{a, A, q, x}$ in $\mathbb{B}^{13}-\mathcal{H}$, rel endpoints, and similarly with $y$ or $z$ in place of $x$. By the symmetry just established, the $y$ and $z$ cases follow from the $x$ case. Lemma A.3 describes how the mirrors of $\mathcal{H}$ meet $\triangle \rho q p$. Namely, $x^{\perp}$ contains $\overline{p q}$, the other three mirrors from (1) meet the triangle at $q$ only, and all other mirrors miss it completely. This verifies the hypothesis of lemma 3.5. whose conclusion is that $\mu_{a, A, x} \simeq \mu_{a, A, q, x}$, as claimed.

(3) Let $U$ be an open ball around $q$, small enough that the only mirrors meeting it are the ones through $q$. We also take it small enough to miss $A$. Let $c$ be any point of $\overline{\rho q} \cap U$ other than $q$. Examining the definition of the meridian shows that we may suppose without loss of generality that $c$ is the "turning point" of $\mu_{a, A, q, x}$. Formally: $\mu_{a, A, q, x}$ equals $\overline{a c}$ followed by $\mu_{c, x}$ followed by $R_{x}(\overline{c a})$. (Note that $\overline{a c} \subseteq \overline{\rho q}$ by our choice of $a$ at the beginning of the proof. And we can use $\overline{a c}$ in place of $\vec{a} \bar{c}$ because it misses $\mathcal{H}$ by lemma A.3.) Another way to state the homotopy $\mu_{a, A, x} \simeq \mu_{a, A, q, x}$ of the previous paragraph is that $M_{a, A, x}=\left(\mu_{a, A, x}, R_{x}\right) \in G_{a}$ corresponds to $M_{c, x}=\left(\mu_{c, x}, R_{x}\right) \in G_{c}$ under the identification of $G_{a}$ with $G_{c}$ induced by $\overline{a c}$. And similarly with $y$ or $z$ in place of $x$. Therefore proving (3) is equivalent to proving that $M_{c, z}$ lies in the subgroup of $G_{c}$ generated by $M_{c, x}$ and $M_{c, y}$.

We focus attention on $U$ by defining a "local" analogue of $G_{c}$, namely $J_{c}=$ $\pi_{1}^{\text {orb }}\left((U-\mathcal{H}) /\left\langle R_{x}, R_{y}\right\rangle, c\right)$. By considering the natural map $J_{c} \rightarrow G_{c}$, it suffices to prove that $M_{c, z}$ lies in the subgroup of $J_{c}$ generated by $M_{c, x}$ and $M_{c, y}$. We convert 
this into a problem in the Euclidean space $T_{q} \mathbb{B}^{13}$ as follows. We write $g_{0}$ for the Riemannian metric on $\mathbb{B}^{13}$ got by identifying $\mathbb{B}^{13}$ with $T_{q} \mathbb{B}^{13}$ under the exponential

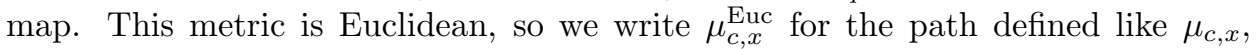
but using this Euclidean metric in place of the hyperbolic metric. We claim: $\mu_{c, x}^{\text {Euc }}$ and $\mu_{c, x}$ are homotopic rel endpoints in $U-\mathcal{H}$. In particular, $M_{c, x}=\left(\mu_{c, x}, R_{x}\right)$ and $M_{c, x}^{\text {Euc }}=\left(\mu_{c, x}^{\text {Euc }}, R_{x}\right)$ are the same element of $J_{c}$, and similarly with $y$ or $z$ in place of $x$. After proving this claim, it will suffice to prove that $M_{c, x}^{\text {Euc }}$ and $M_{c, y}^{\text {Euc }}$ generate $J_{c}$.

To prove the claim, we define for each $t \in(0,1]$ a diffeomorphism $S_{t}$ of $\mathbb{B}^{13}$, namely the one corresponding to the scaling-by- $t$ map on $T_{q} \mathbb{B}^{13}$. Let $g_{t}$ be the Riemannian metric got by pulling back the hyperbolic metric under $S_{t}$ and multiplying it by $1 / t$. It is easy to see that the $g_{t}$ converge to $g_{0}$ as $t \rightarrow 0$. For any $t \in[0,1]$ we define $\mu_{c, x}^{t}$ just as we did for $\mu_{c, x}$, except that we use $g_{t}$ in place of the hyperbolic metric. All the ingredients in this definition (nearest points, geodesics, etc.) vary continuously with $t$, so the $\mu_{c, x}^{t}$ provide a homotopy between $\mu_{c, x}^{1}=\mu_{c, x}$ and $\lim _{t \rightarrow 0} \mu_{c, x}^{t}=\mu_{c, x}^{\text {Euc }}$, as desired. (Note that $\mathbb{B}^{13}$ is nonpositively curved for any $t$; for $t>0$ this is because $\left(\mathbb{B}^{13}, g_{t}\right)$ is isometric to $\mathbb{B}^{13}$ with its metric scaled, and scaling doesn't affect the sign of sectional curvature. And $g_{0}$ is Euclidean by definition. $U$ is convex under any $g_{t}$, because it is always an open ball centered at $q$.)

It remains to show that $M_{c, z}^{\text {Euc }}$ lies in the group generated by $M_{c, x}^{\text {Euc }}$ and $M_{c, y}^{\text {Euc }}$. Obviously we may restrict attention to the $\mathbb{B}^{2}$ orthogonal to $\langle x, y\rangle^{\perp}$ at $q$. We will identify $T_{q} \mathbb{B}^{2}$ with the $\mathbb{C}^{2}$ from lemma 5.2 in a way which lets us quote that result. We first note that $\mathbb{B}^{2}=\mathbb{B}(V)$ where $V$ is the complex span of $\rho, x$ and $y$. We write $W$ for the subspace of $V$ spanned by $x$ and $y$, and $Q$ for the linear projection of $\rho$ to $W^{\perp}$. This is a vector representing $q \in \mathbb{B}^{2}$. We identify $\mathbb{B}^{2}$ with a neighborhood of 0 in $W$ by

$$
(w \in W) \leftrightarrow(\text { the image in } P V \text { of } Q+w) .
$$

Up to a scaling factor, this correspondence is essentially the exponential map of $\mathbb{B}^{13}$ at $q$. This yields an identification of $T_{q} \mathbb{B}^{2}$ with $T_{0} W=W$, in which the mirrors through $q$ correspond to the mirrors of the $D_{4}^{\mathcal{E}}$ spanned by $x$ and $y$, and this identification is equivariant under the action of the finite group generated by $R_{x}$ and $R_{y}$. (We saw in (2) that no more mirrors pass through q.) Furthermore, $c$ lies in $P(\mathbb{C} Q+\mathbb{C} \rho)$, whose corresponding complex line in $W$ is spanned by the projection $\pi_{W}(\rho)$ of $\rho$ to $W$. This projection can be worked out from $\langle\rho \mid x\rangle=\langle\rho \mid y\rangle=\theta$, namely $\pi_{W}(\rho)=\omega x-\bar{\omega} y$.

Now we identify $x$ (resp. $y$ ) with $\alpha$ (resp. $\beta$ ) in lemma 5.2. Under this identification, $T_{q} \mathbb{B}^{2}-T_{q} \mathcal{H}$ is identified with the mirror complement of $D_{4}^{\mathcal{E}}$, with $c$ corresponding to some scalar multiple of $\omega \alpha-\bar{\omega} \beta=(-1,-\bar{\omega} \sqrt{2})$. That lemma tells us that $M_{c, \alpha}$ and $M_{c, \beta}$ generate $\pi_{1}^{\text {orb }}\left(\left(\mathbb{C}^{2}-\mathcal{H}\right) /\left\langle R_{\alpha}, R_{\beta}\right\rangle, c\right)$. Transferring this back to $U-\mathcal{H}$ finishes the proof.

Lemma 5.5. Suppose $x, z$ are Leech roots with $\langle x \mid z\rangle=-\frac{3}{2}+\frac{\theta}{2}$, and let $q$ be the projection of $\rho$ to the intersection of their mirrors. Then

(1) Of the four mirrors of the reflection group $\left\langle R_{x}, R_{z}\right\rangle$, two are Leech mirrors $\left(x^{\perp}\right.$ and $\left.z^{\perp}\right)$, one is a second-shell mirror $\left(y^{\perp}\right.$ for $\left.y=\bar{\omega} x-z\right)$, and one is a third-shell mirror.

(2) These mirrors are the only ones containing $q$. 
(3) The subgroup of $G_{a}$ generated by the Leech meridian $M_{a, A, x}$ and the secondshell meridian $M_{a, A, y}$ also contains the Leech meridian $M_{a, A, z}$.

Proof. This is very similar to the proof of lemma 5.4. By lemma 5.3 we may suppose without loss of generality that $x=(0 ; 1,-\omega)$ and $z=\left(\lambda_{9} ; 1, \theta\right)$, and as before we take $a \in \overline{\rho q}$.

(11) Using $x^{2}=z^{2}=3$ and $\langle x \mid z\rangle=-\frac{3}{2}+\frac{\theta}{2}$, one checks that $y$ and $x+z$ are roots. So the 24 roots in $\langle x, z\rangle$ are their unit multiples together with those of $x$ and $z$. Using $\langle\rho \mid x\rangle=\langle\rho \mid z\rangle=\theta$, one computes $\langle\rho \mid y\rangle=3 \bar{\omega}$ and $\langle\rho \mid x+z\rangle=2 \theta$. So $y$ is a second shell root and $x+z$ is a third shell root.

(2) This is just like the corresponding part of the previous lemma, except that we refer to lemma A.4 in place of lemma A.3

In preparation for (3), we claim that $\mu_{a, A, x}$ is homotopic to $\mu_{a, A, q, x}$ in $\mathbb{B}^{13}-\mathcal{H}$, rel endpoints, and similarly with $y$ or $z$ in place of $x$. This is just like the corresponding part of the previous proof, except that there is no cyclic symmetry. So one has to analyze three triangles rather than just one. This is done in lemma A.4.

(3) This is just like the corresponding part of the previous lemma. The only difference is in the very last step: now $\langle\rho \mid x\rangle=\theta$ and $\langle\rho \mid y\rangle=3 \bar{\omega}$, so $\pi_{W}(\rho)=$ $-\frac{\theta}{2} \omega x+\left(\frac{\theta}{2}-1\right) \omega y$. We still identify $T_{q} \mathbb{B}^{2}$ with $\mathbb{C}^{2}$ by taking $x$ and $y$ to correspond to lemma [5.2] $\mathrm{s} \alpha$ and $\beta$. This makes sense since one can check $\langle x \mid y\rangle=\theta$. Under this identification, $\pi_{W}(\rho)$ corresponds to $\frac{\omega}{2}(\theta+1,(\theta-2) \sqrt{2}) \in \mathbb{C}^{2}$, and we can apply lemma 5.2 just as before.

Lemma 5.6. Suppose $x$ is a point-root and $y$ is an incident line-root. Then $z=$ $\bar{\omega} x-y$ is a Leech root, and the subgroup of $G_{a}$ generated by $M_{a, A, x}$ and $M_{a, A, y}$ contains the Leech meridian $M_{a, A, z}$.

Proof. Using $x^{2}=y^{2}=3$ and $\langle x \mid y\rangle=\theta$, one checks $z^{2}=3$, so $z$ is a root. Using $\langle\rho \mid x\rangle=\theta$ and $\langle\rho \mid y\rangle=3 \bar{\omega}$, one checks $\langle\rho \mid z\rangle=\theta$, so $z$ is a Leech root. Similarly, one checks that $\langle x \mid z\rangle=-\frac{3}{2}+\frac{\theta}{2}$. So we may apply lemma 5.5 to $x$ and $z$. One checks that the root called $y$ there is the same as the one we have called $y$. To finish the proof we appeal to lemma 5.5(3).

Proof of theorem 5.1. Let $G$ denote the subgroup of $G_{a}$ generated by the 26 point and line meridians $M_{a, A, s}$ based at $a$. We must prove $G=G_{a}$. By theorem 4.1 it suffices to show that $G$ contains the Leech meridians associated to the Leech roots $p_{i}, \bar{\omega} p_{i}-l_{j}, p_{i}-\rho$ and $\bar{\omega} p_{i}-l_{j}-\rho$, where $i, j=1, \ldots, 13$ and $p_{i}$ and $l_{j}$ are incident. $G$ contains the Leech meridians associated to the $p_{i}$ by definition, and those associated to the $\bar{\omega} p_{i}-l_{j}$ by lemma 5.6. By $L_{3}(3)$ symmetry, it now suffices to show that $G$ contains the meridians corresponding to the Leech roots $p_{1}-\rho$ and $\bar{\omega} p_{1}-l_{1}-\rho$.

For $p_{1}-\rho$, consider the following sequence of Leech roots:

$$
\begin{array}{lll}
s_{1}=\bar{\omega} p_{1}-l_{1} & s_{7}=-\bar{\omega} s_{1}-\omega p_{4} & s_{13}=-\bar{\omega} s_{12}-\omega s_{11} \\
s_{2}=\bar{\omega} p_{1}-l_{11} & s_{8}=-\bar{\omega} s_{4}-\omega p_{3} & s_{14}=-\bar{\omega} s_{13}-\omega p_{1} \\
s_{3}=\bar{\omega} p_{1}-l_{13} & s_{9}=-\bar{\omega} s_{4}-\omega p_{5} & s_{15}=-\bar{\omega} s_{3}-\omega s_{14} \\
s_{4}=\bar{\omega} p_{2}-l_{2} & s_{10}=-\bar{\omega} s_{5}-\omega s_{8} & s_{16}=-\bar{\omega} s_{15}-\omega s_{7} \\
s_{5}=\bar{\omega} p_{5}-l_{5} & s_{11}=-\bar{\omega} s_{6}-\omega s_{9} & s_{17}=-\bar{\omega} s_{16}-\omega p_{10} \\
s_{6}=\bar{\omega} p_{11}-l_{11} & s_{12}=-\bar{\omega} s_{2}-\omega s_{10} &
\end{array}
$$


In each equation in the left column, the point and line root on the right hand side are incident. By lemma 5.6, $G$ contains the Leech meridian associated to the Leech root defined by that equation. In each equation in the other two columns, the roots appearing on the right side are Leech roots and $\langle$ the first one $|$ the second $\rangle=\theta$. By repeated use of lemma 5.4(3), $G$ contains the meridians associated to the Leech roots defined by these equations. And one checks that $s_{17}=p_{1}-\rho$.

For $\bar{\omega} p_{1}-l_{1}-\rho$ the argument is the same, defining

$$
\begin{aligned}
& s_{1}=\bar{\omega} p_{3}-l_{2} \quad s_{6}=-\bar{\omega} s_{1}-\omega p_{5} \quad s_{11}=-\bar{\omega} s_{8}-\omega s_{10} \\
& s_{2}=\bar{\omega} p_{6}-l_{5} \quad s_{7}=-\bar{\omega} s_{5}-\omega p_{12} \quad s_{12}=-\bar{\omega} s_{2}-\omega s_{11} \\
& s_{3}=\bar{\omega} p_{8}-l_{5} \quad s_{8}=-\bar{\omega} s_{4}-\omega s_{6} \quad s_{13}=-\bar{\omega} s_{12}-\omega s_{1} \\
& s_{4}=\bar{\omega} p_{2}-l_{6} \quad s_{9}=-\bar{\omega} s_{3}-\omega s_{7} \quad s_{14}=-\bar{\omega} s_{13}-\omega p_{11} \\
& s_{5}=\bar{\omega} p_{2}-l_{12} \quad s_{10}=-\bar{\omega} s_{9}-\omega p_{13}
\end{aligned}
$$

and checking that $s_{14}=\bar{\omega} p_{1}-l_{1}-\rho$. We found these sequences of Leech roots by a rather intensive computer search. But their validity can be verified easily.

\section{Change of Basepoint}

In this section we prove the main theorem of the paper, theorem 1.2 the orbifold fundamental group $G_{\tau}=\pi_{1}^{\text {orb }}\left(\left(\mathbb{B}^{13}-\mathcal{H}\right) / P \Gamma, \tau\right)$ is generated by the 26 pointand line-meridians $M_{\tau, H}$. Here $\tau=(4+\sqrt{3} ; 1, \ldots, 1)$ is the 26-point specified in section 2.6 and $H$ varies over the point- and line-mirrors. The starting point of the proof is theorem [5.1] for any basepoint $a$ in the horoball $A$ centered at the Leech cusp $\rho$, the 26 point- and line-meridians $M_{a, A, H}$ generate $G_{a}$. Here $\rho=(3 \omega-1 ;-1, \ldots,-1)$ is the Leech cusp defined in section 2.8 and used throughout section [5, and $H$ varies over the same 26 mirrors. By remark 3.3 we may choose $a$ to be a point of $\overline{\tau \rho}$ very close to $\rho$; exactly how close will be specified later. So $\overline{\tau a}$ is a subsegment of $\overline{\tau \rho}$. In light of theorem 5.1, theorem 1.2 follows immediately from the next lemma.

Lemma 6.1. Suppose $H$ is a point- or line-mirror. Then the meridian $M_{\tau, H} \in G_{\tau}$ corresponds to the meridian $M_{a, A, H} \in G_{a}$ under the isomorphism $G_{\tau} \cong G_{a}$ induced by the path $\overline{\tau a}$.

For the lemma to make sense, one must verify that $\overline{\tau a}$ misses $\mathcal{H}$. Because $\overline{\tau a} \subseteq \overline{\tau \rho}$, this follows from the stronger result (lemma B.2) that the complex triangle $\triangle \rho \tau l_{\infty}$ misses $\mathcal{H}$ except at $l_{\infty}$.

Proof. We will give the proof when $H$ is a line-mirror, and then remark on the changes needed for the point-mirror case. Recall from section 2.6 that $l_{\infty}$ is where all 13 line-mirrors intersect. We will use a 4-step homotopy. The first step corresponds to the bottom region (shaded darkly) in figure 6.1, the second step to the region above it (lighter), the third to the next (dark again), and the fourth to the rightmost region (light again). The key fact is that these regions miss $\mathcal{H}$ except at known points. These verifications are lemmas A.6 A.13 and B.2 in the appendices.

The first step is that $M_{\tau, H}$ is equal to $M_{\tau, l_{\infty}, H}$ in $G_{\tau}$. This follows from lemma 3.6 once one checks that $\triangle \tau p l_{\infty}$ misses $\mathcal{H}$, except that $\overline{p l_{\infty}}$ lies in $H$ and $l_{\infty}$ may lie in additional hyperplanes. Here $p$ means the point of $H$ closest to $\tau$. This check is lemma A.6. 


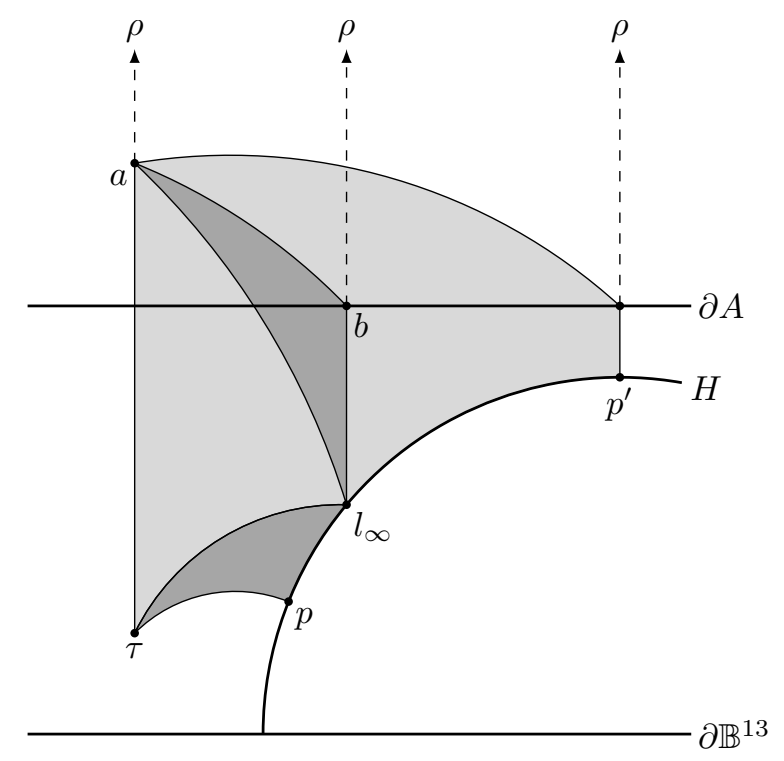

FiguRE 6.1. The regions correspond to the four steps in the proof of lemma 6.1 starting from the bottom. We use the upper half space model with the Leech cusp $\rho$ at vertical infinity and $A$ being the horoball centered there, whose boundary is the horizontal line. Each of the regions misses $\mathcal{H}$ except for obvious intersection points.

The second step is that the isomorphism $G_{\tau} \cong G_{a}$ identifies $M_{\tau, l_{\infty}, H}$ with $M_{a, l_{\infty}, H}$. This follows from lemma 3.2, once one checks that the (complex) triangle $\triangle a \tau l_{\infty}$ misses $\mathcal{H}$ except at $l_{\infty}$. This check is lemma B.2, it proves the corresponding result for $\triangle \rho \tau l_{\infty}$, which contains $\triangle a \tau l_{\infty}$.

The third step is that $M_{a, l_{\infty}, H}$ is equal to $M_{a, A, l_{\infty}, H}$ in $G_{a}$. By taking $a$ very far up in figure 6.1 this becomes almost obvious. Namely, the uniform distance between $\overline{a l_{\infty}}$ and the concatenation of $\overline{a b}$ and $\overline{b l_{\infty}}$ tends to 0 as $a$ approaches $\rho$. Here $b$ is the point of $A$ closest to $l_{\infty}$. Therefore, by taking $a$ close enough to $\rho$, we may take the uniform distance between $M_{a, l_{\infty}, H}$ and $M_{a, A, l_{\infty}, H}$ to be arbitrarily small. We take it small enough that the straight-line homotopy misses $\mathcal{H}$ (This uses the fact verified in lemma A.13 that the geodesic segment joining $b$ and $l_{\infty}$ misses $\mathcal{H}$ except at $l_{\infty}$.)

The final step is like the first: $M_{a, A, l_{\infty}, H}$ is equal in $G_{a}$ to $M_{a, A, H}$. This follows from lemma 3.5, once one checks that $\triangle \rho l_{\infty} p^{\prime}$ misses $\mathcal{H}$ except that $\overline{p^{\prime} l_{\infty}} \subseteq H$ and that $l_{\infty}$ may lie in additional mirrors. Here $p^{\prime}$ is the point of $\mathcal{H}$ nearest $\rho$. This check is lemma A.13.

Putting these four steps together gives

$$
M_{\tau, H}=M_{\tau, l_{\infty}, H} \leftrightarrow M_{a, l_{\infty}, H}=M_{a, A, l_{\infty}, H}=M_{a, A, H}
$$

Here the first two terms are equal in $G_{\tau}$ by the first step of the homotopy. The second and third terms correspond under the isomorphism $G_{\tau} \cong G_{a}$ induced by $\overline{\tau a}$, by the second step. And the last three terms are equal in $G_{a}$ by the third and fourth steps. This finishes the proof for line-meridians. 
The point-meridian case is exactly the same. Now $H$ is a point-mirror and $p$ and $p^{\prime}$ are the projections of $\tau$ and $\rho$ to it. And we replace $l_{\infty}$ by $p_{\infty}$. In step $1, \triangle \tau p l_{\infty}$ gets replaced by $\triangle \tau p p_{\infty}$, whose intersection with $\mathcal{H}$ is also given by lemma A.6. In step $2, \triangle \tau \rho l_{\infty}$ gets replaced by $\triangle \tau \rho p_{\infty}$, whose intersection with $\mathcal{H}$ is also given by lemma B.2. In step $4, \triangle \rho p^{\prime} l_{\infty}$ gets replaced by $\triangle \rho p^{\prime} p_{\infty}$, whose intersection with $\mathcal{H}$ is given by lemma A.11 rather than lemma A.13.

\section{Appendix A. How eight totally REAl triangles MeEt the MirRors}

In this appendix we examine how certain totally real triangles in $\overline{\mathbb{B}^{13}}$ meet the mirror arrangement $\mathcal{H}$. This was needed in the proofs of lemmas 5.4, 5.5 and 6.1. Recall from section 2.3 that if $x, y, z \in L \otimes \mathbb{C}-\{0\}$ have norms and inner products in $(-\infty, 0]$, then the convex hull of the corresponding points in $\overline{\mathbb{B}^{13}}$ is the projectivization of the convex hull of $x, y$ and $z$ in $L \otimes \mathbb{C}$. In section 2.3 we called such a triangle in $\overline{\mathbb{B}^{13}}$ totally real. For totally real triangles we don't usually distinguish between the triangle in $L \otimes \mathbb{C}$ and the triangle in $\overline{\mathbb{B}^{13}}$. The following lemma is trivial but crucial.

Lemma A.1 (How to intersect a totally real triangle and a hyperplane). Suppose $x, y, z \in L \otimes \mathbb{C}$ have norms and inner products in $(-\infty, 0], T$ is the totally real triangle $\triangle x y z$, and $s \in L \otimes \mathbb{C}$. Then $T \cap s^{\perp}$ is the preimage of the origin under the map $T \rightarrow \mathbb{C}$ given by $v \mapsto\langle v \mid s\rangle$.

In particular, if the convex hull in $\mathbb{C}$ of $\langle x \mid s\rangle,\langle y \mid s\rangle$ and $\langle z \mid s\rangle$ does not contain the origin, then $T \cap s^{\perp}=\emptyset$.

In the situation of the lemma we will write $\langle T \mid s\rangle$ for the convex hull in $\mathbb{C}$ of $\langle x \mid s\rangle,\langle y \mid s\rangle$ and $\langle z \mid s\rangle$. Note that if the vertices of the triangle $\langle T \mid s\rangle$ have coordinates in $\mathbb{Q}[\omega]$ or $\mathbb{Q}[\omega, \sqrt{3}]$, then the condition $0 \in\langle T \mid s\rangle$ can be checked by exact arithmetic in that field. Most arguments in this appendix amount to showing that $0 \notin\langle T \mid s\rangle$ for various triangles $T$ and roots $s$. Some of the results require computer calculation, and others depend on properties of the Leech lattice. For example, lemmas A.3 and A.13 use the following result:

Lemma A.2 (Leech lattice points near $\lambda_{6} / \theta$ and $\left.\lambda_{9} / \theta\right)$. Suppose $\lambda_{6}$ and $\lambda_{9}$ are vectors in $\Lambda$ with norms 6 and 9 . Then the nearest points of $\Lambda$ to $\lambda_{6} / \theta$ are three in number, at distance $\sqrt{2}$. And the nearest points of $\Lambda$ to $\lambda_{9} / \theta$ are 36 in number, at distance $\sqrt{3}$.

Proof. The essential point is that the shortest elements of $\Lambda$ which are congruent $\bmod \theta$ to a lattice vector of norm 6 (resp. 9) are 3 (resp. 36) in number, all of norm 6 (resp. 9). This is the lemma on p. 153 of $\mathrm{W}$.

We abbreviate $\lambda_{6} / \theta$ to $C$ (for "centroid"). $\Lambda$ contains the vector $C-\lambda_{6} / \theta$, which lies at distance $\sqrt{2}$ from $C$. (It lies in $\Lambda$ because it is the zero vector.) Now consider any lattice point at distance $\leq \sqrt{2}$ from $C$, and write it as $C+x / \theta$ with $x \in \Lambda \otimes \mathbb{C}$ of norm $\leq 6$. Since $\Lambda$ contains the difference between this point and the one just mentioned, it contains $\left(x+\lambda_{6}\right) / \theta$. That is, $x$ lies in $\Lambda$ and is congruent to $-\lambda_{6} \bmod \theta$. By Wilson's result, the possibilities for $x$ are the three minimal representatives of $-\lambda_{6}$ 's congruence class $\bmod \theta$. This finishes the $\lambda_{6}$ case. The $\lambda_{9}$ case is similar.

When we have a Leech cusp $\rho$ in mind, we will call a horosphere centered there a critical horosphere if it is tangent to some mirror. We use the same language for 
the (open or closed) horoball it bounds. Recall the definition of a horosphere of height $h$ given in the discussion following equation (2.3). If $s$ is a root then it is easy to see that the horosphere tangent to its mirror is the one of height $\frac{1}{3}|\langle\rho \mid s\rangle|^{2}$. (Compute the inner product of $\rho$ with its projection onto $s^{\perp}$.) So the heights of the first four critical horospheres are 1, 3, 4 and 7. The first-, second- and third-shell mirrors (defined in section 2.8) are the mirrors tangent to the first, second and third critical horoballs.

The following lemma is the first of several in this appendix having the general form: prove that some particular triangle misses the mirrors except for some obvious intersection points. The general strategy is to start by showing that the triangle is covered by the union of a ball and a horoball. We enumerate the finitely many mirrors that meet the ball, and check by direct computation that they miss the triangle. Then we enumerate the mirrors that meet the horoball; there are infinitely many such mirrors, but they correspond to roots of small height and therefore they may be parametrized. Checking that these mirrors miss the triangle requires some intricate analysis rather than just direct computation.

Lemma A.3 (The triangle needed in lemma 5.4). In the Leech model, consider the Leech roots $x=(0 ; 1,-\omega)$ and $y=\left(\lambda_{6} ; 1, \omega\right)$, where $\lambda_{6}$ is a norm 6 vector in $\Lambda$. Define $p$, resp. $q$, as the projection of $\rho$ to $x^{\perp}$, resp. $\langle x, y\rangle^{\perp}$. Then the totally real triangle $T=\triangle \rho p q$ meets $\mathcal{H}$ as follows: $x^{\perp}$ meets $T$ in $\overline{p q}$, the other three mirrors of $\left\langle R_{x}, R_{y}\right\rangle$ meet $T$ only at $q$, and all other mirrors miss $T$.

Proof. First we introduce various important points. Recall that $\rho=(0 ; 0,1)$. By definition,

$$
p=\rho-\frac{\langle\rho \mid x\rangle}{x^{2}} x=(0 ; 1 / \theta,-\bar{\omega} / \theta),
$$

and one can compute $p^{2}=\langle\rho \mid p\rangle=-1$. For later calculations, we also write $p$ in the form (2.6), writing $\sigma_{p}, m_{p}, N_{p}$ and $\nu_{p}$ in place of their unsubscripted forms that appear there. Obviously we have $\sigma_{p}=0$ and $m_{p}=1 / \theta$. And $N_{p}$ is $p^{2}$, which we just computed to be -1 . One can then solve for $\nu_{p}$, namely $\nu_{p}=-\frac{\theta}{18}$. One can verify the following formula for $q$ by checking that it is orthogonal to $x$ and $y$ :

$$
q=\rho-(\omega x-\bar{\omega} y)=\left(\bar{\omega} \lambda_{6} ; \bar{\theta}, \theta \bar{\omega}\right) .
$$

One can check $q^{2}=\langle\rho \mid q\rangle=\langle p \mid q\rangle=-3$. Since the vectors we have chosen to represent the three vertices of $\triangle \rho p q$ have inner products in $(-\infty, 0]$, we will be able to apply lemma A.1. Also, writing $q$ in the form (2.6), just as we did for $p$, gives $\sigma_{q}=\bar{\omega} \lambda_{6}, m_{q}=\bar{\theta}, N_{q}=-3$ and $\nu_{q}=-\frac{\theta}{2}$.

The mirrors of $\left\langle R_{x}, R_{y}\right\rangle$ obviously meet $T$ as claimed, so it suffices to show that no other mirrors meet $T$. By construction, $p$ lies on the boundary of the first critical horoball. And $\operatorname{ht}(q)=3$, so $q$ lies on the boundary of the second. Therefore only one mirror not in the first shell could meet the triangle, and then only at $q$. This mirror corresponds to the second-shell root $\omega x-\bar{\omega} y$ in $\langle x, y\rangle$ that we left unnamed in lemma 5.4(1). So it suffices to show that no Leech mirrors meet the triangle except the three coming from $\langle x, y\rangle$.

Our strategy is to write a general Leech root $s$ in the form (2.8), namely

$$
s=\left(\sigma ; 1, \theta\left(\frac{\sigma^{2}-3}{6}+\nu\right)\right)
$$


and compute its inner products with $\rho, p$ and $q$, and then apply lemma A.1 to gain control over $\sigma$ and $\nu$. To find $\langle p \mid s\rangle$ and $\langle q \mid s\rangle$ we appeal to formula (2.7); being able to apply this is the reason we computed $\sigma_{p}, \ldots, \nu_{q}$. By (2.7), we have

$$
\begin{aligned}
& \langle p \mid s\rangle=\frac{\theta}{6} \sigma^{2}+\left(\frac{1}{2}-\theta \nu\right) \\
& \langle q \mid s\rangle=\frac{\theta}{2}\left(\left(\sigma+\bar{\omega} \frac{\lambda_{6}}{\theta}\right)^{2}-2\right)-\left(\theta \operatorname{Im}\left\langle\sigma \mid \bar{\omega} \frac{\lambda_{6}}{\theta}\right\rangle-\frac{3}{2}+3 \theta \nu\right)
\end{aligned}
$$

In each of these, the first term is imaginary and the second is real. Our goal is to show that only three possible pairs $\sigma, \nu$ allow 0 to lie in the triangle $\langle T \mid s\rangle \subseteq \mathbb{C}$ whose vertices are (A.1), A.2 and $\langle\rho \mid s\rangle=\theta$.

First we consider the case $\sigma=0$. Then A.1 and A.2 simplify dramatically, and $\langle T \mid s\rangle$ is the triangle in $\mathbb{C}$ with vertices $\frac{1}{2}-\theta \nu, 3\left(\frac{1}{2}-\theta \nu\right)$ and $\theta$. Since the last vertex is above the real axis, while the first two are real and differ by a factor of 3 , $\langle T \mid s\rangle$ can only contain 0 if both of its first two vertices are 0 . This forces $\nu=1 / 2 \theta$, which leads to $s=x$, whose mirror we already know meets $T$.

Now we suppose $\sigma \neq 0$. So (A.1) lies above the real axis, just as $\langle\rho \mid s\rangle$ does. If $\sigma$ lies at distance $>\sqrt{2}$ from $-\bar{\omega} \lambda_{6} / \theta$ then (A.2) also lies above the real axis. In this case it is obvious that $0 \notin\langle T \mid s\rangle$. By lemma A.2, the only other possibility is that $\sigma$ is one of the three elements of $\Lambda$ that lie at distance $\sqrt{2}$ from $-\bar{\omega} \lambda_{6} / \theta$, in which case $\langle q \mid s\rangle$ is real. One of these nearest neighbors is $0 \in \Lambda$, which we treated in the previous paragraph. In each of the other two cases, $\langle p \mid s\rangle$ and $\langle\rho \mid s\rangle$ are still above the $x$-axis and $\langle q \mid s\rangle$ is on the $x$-axis. So the only way the origin can lie in $\langle T \mid s\rangle$ is for it to be the vertex $\langle q \mid s\rangle$. Then $\nu$ is determined in terms of $\sigma$ using equation (A.2), since $\langle q \mid s\rangle=0$. We have shown that there are at most three Leech mirrors that meet the triangle. Since we know three Leech mirrors that do meet it, coming from roots in $\langle x, y\rangle$, the proof is complete.

Lemma A.4 (Three triangles needed in lemma 5.5). In the Leech model, consider the Leech roots $x=(0 ; 1,-\omega)$ and $z=\left(\lambda_{9} ; 1, \theta\right)$ and define the second shell root $y$ as $\bar{\omega} x-z$. Write $X, Y$ and $Z$ for the projections of $\rho$ to $x^{\perp}, y^{\perp}$ and $z^{\perp}$, and $q$ for the projection of $\rho$ to $\langle x, y\rangle^{\perp}$. Then the only mirrors of $\mathcal{H}$ which meet any of the totally real triangles $\triangle \rho q X, \triangle \rho q Y$ and $\triangle \rho q Z$ are the four mirrors of $\left\langle R_{x}, R_{y}\right\rangle$.

Proof. We begin by finding basic data about various important points. In addition to $x$ and $z$ given in the statement, we have

$$
\rho=(0 ; 0,1) \quad \text { and } \quad y=\left(-\lambda_{9} ; \theta \omega, 2 \bar{\omega}\right)
$$

where $\lambda_{9}$ is some norm 9 vector in the Leech lattice. Recall that $x$ and $z$ are Leech roots, so $\langle\rho \mid x\rangle=\langle\rho \mid z\rangle=\theta$. Also, $\langle x \mid z\rangle=-\frac{3}{2}+\frac{\theta}{2}$ and $y$ is a second-shell root with $\langle\rho \mid y\rangle=3 \bar{\omega}$. The projections $X, Y, Z$ are

$$
\begin{aligned}
& X=\rho-\frac{\langle\rho \mid x\rangle}{x^{2}} x=\rho+x / \theta=(0 ; 1 / \theta,-\bar{\omega} / \theta) \\
& Y=\rho-\frac{\langle\rho \mid y\rangle}{y^{2}} y=\rho-\bar{\omega} y=\left(\bar{\omega} \lambda_{9} ; \bar{\theta}, 1-2 \omega\right) \\
& Z=\rho-\frac{\langle\rho \mid z\rangle}{z^{2}} z=\rho+z / \theta=\left(\lambda_{9} / \theta ; 1 / \theta, 2\right)
\end{aligned}
$$

Using these one can check

$$
X^{2}=Z^{2}=-1 \quad\langle\rho \mid X\rangle=\langle\rho \mid Z\rangle=-1 \quad\langle X \mid y\rangle=1+3 \bar{\omega}
$$




$$
Y^{2}=-3 \quad\langle\rho \mid Y\rangle=-3 \quad\langle Y \mid x\rangle=-\omega \theta .
$$

Because $X \perp x$ and $Y \perp y$, the following gives a formula for the projection $q$ of $\rho$ to $\langle x, y\rangle^{\perp}$ :

$$
q=(2 \omega-1)\left(\rho-\frac{\langle\rho \mid x\rangle}{\langle Y \mid x\rangle} Y-\frac{\langle\rho \mid y\rangle}{\langle X \mid y\rangle} X\right)=\left((2 \bar{\omega}-\omega) \lambda_{9} ; 3 \bar{\theta}, 3-3 \omega\right)
$$

(The initial factor $2 \omega-1$ makes $\langle\rho \mid q\rangle$ negative, and also makes $q$ a primitive lattice vector.) Using this one can check

$$
q^{2}=-18 \text { and }\langle q \mid \rho\rangle=\langle q \mid X\rangle=\langle q \mid Y\rangle=\langle q \mid Z\rangle=-9
$$

We will be able to apply lemma A.1 to $\triangle \rho q X$ because $\rho, q$ and $X$ have negative inner products. And similarly with $Y$ or $Z$ in place of $X$.

We first claim that $\triangle \rho q X \cup \triangle \rho q Y \cup \triangle \rho q Z$ lies in the interior of the fourth critical horoball. It suffices to show that $X, Y, Z$ and $q$ do. By definition, $Y$ lies on the boundary of the 2nd critical horoball and $X$ and $Z$ lie on the boundary of the 1st. For $q$, we use $q^{2}=-18$ and $\langle q \mid \rho\rangle=-9$ to get $\operatorname{ht}(q)=9 / 2<7$, as desired.

Let $Q_{X}$ be $\triangle \rho q X$ minus the first (open) critical horoball around $\rho$, and similarly for $Q_{Y}$ and $Q_{Z}$. By working in the hyperbolic plane containing $\triangle \rho q X$, it is obvious that the point of $Q_{X}$ furthest from $q$ is $X$. The distance formula (2.1) gives $d(q, X)=\cosh ^{-1} \sqrt{9 / 2}$. Similarly, $Z$ is the point of $Q_{Z}$ furthest from $q$, and $d(q, Z)$ is also $\cosh ^{-1} \sqrt{9 / 2}$. Finally, the furthest point of $Q_{Y}$ from $q$ is where $\overline{Y \rho}$ intersects the first critical horosphere. We can find this point by parameterizing $\overline{Y \rho}-\{\rho\}$ by $Y+t \rho$ for $t \in[0, \infty)$. The intersection point is defined by the condition ht $_{\rho}(Y+t \rho)=1$. Writing this out explicitly using (2.3) and solving for $t$ gives $t=1$. So the point is $Y+\rho$. The distance formula gives $d(q, Y+\rho)=\cosh ^{-1} \sqrt{2}$. This is smaller than $\cosh ^{-1} \sqrt{9 / 2}$, so we conclude that $Q_{X} \cup Q_{Y} \cup Q_{Z}$ lies in the closed ball around $q$ of radius $\cosh ^{-1} \sqrt{9 / 2}$.

Now suppose $s$ is a root whose mirror meets $\triangle \rho q X \cup \triangle \rho q Y \cup \triangle \rho q Z$. Since these triangles lie in the fourth (open) critical horoball, $s$ must be a 1st, 2nd or 3rd shell root. By scaling $s$ we may suppose $\langle\rho \mid s\rangle \in\{\theta, 3,2 \theta\}$. We will work with many projections of $s$, so we name them in a uniform way:

$$
\begin{array}{cl}
s_{q}=\frac{\langle s \mid q\rangle}{q^{2}} q & \text { is its projection to the span of } q, \\
s_{q \perp}=s-s_{q} & \text { is its projection to the orthogonal complement of this, } \\
s_{x y} & \text { is its projection to the span of } x \text { and } y, \\
s_{q x y}=s_{q}+s_{x y} & \text { is its projection to the span of } q, x \text { and } y, \\
s_{q x y \perp}=s-s_{q x y} & \text { is its projection to the orthogonal complement of this, } \\
s_{\rho x} & \text { is its projection to the span of } \rho \text { and } x, \\
s_{9} & \text { is its projection to the span of } \lambda_{9} \in \Lambda, \text { and } \\
s_{\Lambda}=s_{9}+s_{q x y \perp} & \text { is its projection to the summand } \Lambda=\langle\rho, x\rangle^{\perp} \text { of } L .
\end{array}
$$

Because all mirrors miss the first (open) critical horoball, $s^{\perp}$ must meet $Q_{X} \cup$ $Q_{Y} \cup Q_{Z}$. Because this set lies in the closed $\left(\cosh ^{-1} \sqrt{9 / 2}\right)$-ball around $q$, we get $d\left(q, s^{\perp}\right) \leq \cosh ^{-1} \sqrt{9 / 2}$. Using the second distance formula (2.2) gives

$$
\sinh ^{-1} \sqrt{-\frac{|\langle q \mid s\rangle|^{2}}{q^{2} s^{2}}} \leq \cosh ^{-1} \sqrt{9 / 2}
$$


Now $s^{2}=3, q^{2}=-18$ and $\sinh \left(\cosh ^{-1}(?)\right)=\sqrt{(?)^{2}-1}$ give us the inequality $|\langle q \mid s\rangle|^{2} \leq 189$. Using $q^{2}=-18$ a second time shows that the most negative $s_{q}^{2}$ can be is $-21 / 2$. Next, $3=s^{2}=s_{q}^{2}+\left(s_{q \perp}\right)^{2}$, so $\left(s_{q \perp}\right)^{2} \leq 27 / 2$. Since $s_{x y}$ is a projection of $s_{q \perp}$ inside the positive definite space $q^{\perp}$, it follows that $s_{x y}^{2} \leq 27 / 2$.

We recall that $\langle x, y\rangle$ is a copy of the Eisenstein lattice $D_{4}^{\mathcal{E}}$, for which one can introduce explicit coordinates, for example as in lemma 5.2. Since all inner products in $L$ are divisible by $\theta$, we have $s_{x y} \in \theta\left(D_{4}^{\mathcal{E}}\right)^{*}$. One can check that $\theta\left(D_{4}^{\mathcal{E}}\right)^{*}$ is a copy of $D_{4}^{\mathcal{E}}$ scaled to have minimal norm $3 / 2$. An $\mathcal{E}$-basis consists of $(\theta x+y) / 2$ and $(\theta y-x) / 2$. Let $S_{x y}$ be the set of vectors in this lattice with norm $\leq 27 / 2$. We have shown that the projection $s_{x y}$ of $s$ must lie in $S_{x y}$. This set can be enumerated on a computer and turns out to have size 937. (PARI has a built-in function to do this. It is most natural to rescale by multiplying all inner products by $4 / 3$, to work with a copy of the standard $D_{4}$ lattice over $\mathbb{Z}$. Then one enumerates all lattice vectors of norm $\leq \frac{4}{3} \cdot \frac{27}{2}=18$. The number 937 matches what one expects from CSI, Table 4.8].)

Now we consider the possibilities for $s_{q x y}$. We claim it lies in

$$
S_{q x y}=\left\{s_{x y}+t q \mid s_{x y} \in S_{x y} \text { and } t=\frac{(\text { one of } \bar{\theta}, 3, \text { and } 2 \bar{\theta})-\left\langle s_{x y} \mid \rho\right\rangle}{-9}\right\}
$$

This is easy: $s_{q x y}$ equals $s_{x y}$ plus some multiple of $q$, and the multiple of $q$ is determined by the condition $\left\langle\rho \mid s_{q x y}\right\rangle=\langle\rho \mid s\rangle \in\{\theta, 3,2 \theta\}$. So $S_{q x y}$ has order $937 \cdot 3=2811$.

We are working under the assumption that $s$ is orthogonal to some point of $Q_{X} \cup Q_{Y} \cup Q_{Z}$. Now, $s_{q x y}$ has the same inner products as $s$ with all elements of the span of $q, x, y$, which contains $Q_{X} \cup Q_{Y} \cup Q_{Z}$. It follows that $s_{q x y}^{\perp}$ meets one of the triangles $\triangle \rho q X, \triangle \rho q Y$ and $\triangle \rho q Z$. Of the 2811 possibilities for $s_{q x y}$, only 460 satisfy this condition. And the following considerations further restrict the possibilities.

Because the span of $q, x$ and $y$ is the same as the span of $\rho, x$ and $\lambda_{9}$ and $\lambda_{9}$ is orthogonal to the span of $\rho$ and $x$, we have $s_{q x y}=s_{9}+s_{x \rho}$ and hence $s=s_{q x y \perp}+s_{9}+s_{x \rho}$. It follows that $s_{\Lambda}=s_{q x y \perp}+s_{9}$. Note that the projection $s_{9}$ of $s$ to $\mathbb{C} \lambda_{9}$ coincides with the corresponding projection of $s_{q x y}$. Furthermore, the equalities

$$
\begin{gathered}
s_{\Lambda}^{2}=\left(s_{q x y \perp}\right)^{2}+s_{9}^{2}=\left(3-s_{q x y}^{2}\right)+s_{9}^{2} \\
\left\langle s_{\Lambda} \mid \lambda_{9}\right\rangle=\left\langle s_{q x y} \mid \lambda_{9}\right\rangle
\end{gathered}
$$

show that $s_{q x y}$ determines $s_{\Lambda}^{2}$ and $\left\langle s_{\Lambda} \mid \lambda_{9}\right\rangle$. Therefore it determines all inner products in the span of $s_{\Lambda}$ and $\lambda_{9}$. Also, note that $s_{\Lambda}$ lies in $\Lambda$, not just $\Lambda \otimes \mathbb{C}$, because $\Lambda$ is an orthogonal summand of $L$.

Of the 460 possibilities for $s_{q x y}$, 449 lead to $\left|\left\langle s_{\Lambda} \mid \lambda_{9}\right\rangle\right|^{2}>9 s_{\Lambda}^{2}$, which violates the Cauchy-Schwarz inequality. Of the remaining 11 possibilities, 4 lead to $s_{\Lambda}^{2}=$ 3 , which contradicts the fact that $\Lambda$ has minimal norm 6 . Of the remaining 7 possibilities, 3 lead to the similar contradiction that $\left(\zeta s_{\Lambda}-\lambda_{9}\right)^{2}=3$ for some unit $\zeta$ of $\mathcal{E}$. The remaining 4 possibilities for $s_{q x y}$ turn out to be $x, \bar{\omega} y, z$ and $x+z$, all of which are roots of $\langle x, y\rangle$. In particular, they all have norm 3 . It follows that

$$
s_{q x y \perp}^{2}=s^{2}-s_{q x y}^{2}=3-3=0 .
$$




$$
\begin{array}{llr}
\operatorname{distance}\left(s^{\perp}, p_{\infty}\right) & \begin{array}{l}
\text { representative } \\
0
\end{array} & \mid 3^{13}: L_{3}(3) \text {-orbit } \mid \\
0 & \left(0 ; 0^{12}\right) & 13 \\
\sinh ^{-1} \sqrt{1 / 3} \approx .549 & \left(1 ; 1^{4}, 0^{9}\right) & 1053=13 \cdot 3^{4} \\
\sinh ^{-1} \sqrt{3 / 3} \approx .881 & \left(\theta ; 1^{3},-1^{3}, 0^{7}\right) & 113724=156 \cdot 3^{6} \\
& \left(\theta ; \theta, \bar{\theta}, 0^{11}\right) & 1404=13 \cdot 12 \cdot 3^{2} \\
& \left(\theta ; \theta, \theta, 0^{11}\right) & 702=\left(\begin{array}{c}
13 \\
2
\end{array}\right) \cdot 3^{2} \\
& \left(\theta ; \bar{\theta}, \bar{\theta}, 0^{11}\right) & 702
\end{array}
$$

TABLE 1 . The $3^{13}: L_{3}(3)$-orbits of batch 0,1 and 2 roots, up to units. Their mirrors pass through or near the 13 -point $p_{\infty}$. The last 13 coordinates, read modulo $\theta$, must give an element of the line code.

Since $s_{q x y \perp}$ lies in the positive-definite space $q^{\perp}$, we conclude $s_{q x y \perp}=0$. Therefore $s=s_{q x y}$, which we have already observed is a root of $\langle x, y\rangle$.

For the rest of the results in this section we will need to understand the mirrors near the intersection point $p_{\infty}$ of all 13 point-mirrors. Just as we spoke of critical horoballs around $\rho$, we will speak of critical balls around $p_{\infty}$. They are defined the same way: the balls centered at $p_{\infty}$ and tangent to some mirror. The radii of the critical balls are called critical radii. Even though some mirrors pass through $p_{\infty}$, we don't regard 0 as a critical radius.

For this analysis we use the $P^{2} \mathbb{F}_{3}$ model, in which $p_{\infty}=(\bar{\theta} ; 0, \ldots, 0)$. For a given root $s$ of $L$, the distance formula (2.2) gives $d\left(p_{\infty}, s^{\perp}\right)$ in terms of $p_{\infty}^{2}=-3$, $s^{2}=3$ and $\left|\left\langle p_{\infty} \mid s\right\rangle\right|^{2}$. Since the inner product lies in $\theta \mathcal{E}$ and hence has norm in $3 \cdot\{0,1,3,4,7,9,12, \ldots\}$, one can work out the critical radii as

$$
r_{1}, r_{2}, r_{3}, r_{4}, r_{5}, r_{6}, \ldots=\sinh ^{-1} \sqrt{(1,3,4,7,9,12, \ldots) / 3}
$$

This assumes that there are indeed roots in $L$ having the appropriate inner products with $p_{\infty}$, which is easy to check in the range we care about (see lemma A.12 below). Numerically, the first six critical radii are approximately $.549, .881, .987,1.210$, 1.317 and 1.444 . We call the mirrors tangent to the $n$th critical ball around $p_{\infty}$ the batch $n$ mirrors. For completeness we also refer to the point-mirrors as the batch 0 mirrors. Lemma A.12 below describes batches $0, \ldots, 5$ explicitly, but until lemma A.13 we only need batches 0,1 and 2. These are easy to enumerate and appear in table 1. As an example application we reprove the following result from Ba1, Prop. 1.2]. Although this result helps motivate the main theorem of the paper (theorem 1.2), it is not logically necessary for us.

Lemma A.5 (Mirrors near a 26-point Ba1, prop. 1.2]). The mirrors of L closest to the 26-point $\tau$ are the point- and line-mirrors, at distance $\sinh ^{-1}(6+8 \sqrt{3})^{-1 / 2} \approx$ .223 .

Proof. First we use (2.1) to compute

$$
d\left(\tau, p_{\infty}\right)=\cosh ^{-1} \sqrt{\frac{\left|\left\langle\tau \mid p_{\infty}\right\rangle\right|^{2}}{p_{\infty}^{2} \tau^{2}}}=\cosh ^{-1} \sqrt{\frac{19+8 \sqrt{3}}{6+8 \sqrt{3}}} \approx .740 .
$$

That the point- and line-mirrors lie at distance $\sinh ^{-1}(6+8 \sqrt{3})^{-1 / 2} \approx .223$ from $\tau$ is a similar calculation, using (2.2) in place of (2.1). Any mirror which passes as 
near or nearer to $\tau$ as these do must lie at distance at most

$$
d\left(\tau, p_{\infty}\right)+\sinh ^{-1}(6+8 \sqrt{3})^{-1 / 2} \approx .740+.223=.963
$$

from $p_{\infty}$. This is less than $r_{3}=\sinh ^{-1} \sqrt{4 / 3} \approx .987$, so all such mirrors occur in batches 0,1 and 2. Our computer iterated over these batches and found that the mirrors closest to $\tau$ are just the point and line mirrors.

Lemma A.6 (Two triangles needed for step 1 of lemma 6.1). Let $s$ be a point-root and $x$ be the projection of the 26-point $\tau$ to its mirror. Then the only mirrors of $L$ that meet the totally real triangle $\triangle \tau x p_{\infty}$ are the point-mirrors. The intersection of $\triangle \tau x p_{\infty}$ with each of them is $p_{\infty}$, except that the intersection with $s^{\perp}$ is $\overline{x p_{\infty}}$. The same results hold if $s$ is a line-mirror, provided we replace $p_{\infty}$ by $l_{\infty}$.

Proof. We prove the point-mirror case; the line-mirror case follows by applying an element of $L_{3}(3): 2$ that swaps the point- and line-mirrors. We gave $d\left(p_{\infty}, \tau\right) \approx .740$ in the previous lemma, and $x$ is closer to $p_{\infty}$ than $\tau$ is. So the triangle lies within $d\left(p_{\infty}, \tau\right)$ of $p_{\infty}$. This is less than $r_{2}=\sinh ^{-1} 1 \approx .881$, so only the batch 0 and 1 mirrors might meet the triangle. The batch 0 mirrors are the point-mirrors, which obviously meet the triangle as stated. For the batch 1 roots we used our computer to check (using lemma A.1) that none of their mirrors meet the triangle. When doing this we replaced the vector $\left(\bar{\theta} ; 0^{13}\right)$ representing $p_{\infty}$ by $\theta p_{\infty}=\left(3 ; 0^{13}\right)$. Then the vectors representing the $\tau, x$ and $p_{\infty}$ have negative inner products, so lemma A.1 applies.

So far we have not needed a concrete description of the Leech lattice. But we need one to check that $\mathcal{H}$ is disjoint from the homotopies in step 4 of the proof of lemma 6.1. We will use Wilson's $L_{3}(3)$-invariant model from [W, p. 188]. Its description involves $\psi=1-3 \bar{\omega} \in \mathcal{E}$ from [W] p. 154], and uses 13 coordinates in $\mathcal{E}$, indexed by the points of $P^{2} \mathbb{F}_{3}$. Namely, $\left(x_{1}, \ldots, x_{13}\right) \in \mathcal{E}^{13}$ lies in $\Lambda$ just if $x_{1}+\cdots+x_{13}=0$, all coordinates are congruent modulo $\bar{\psi}$, and the element of $\mathbb{F}_{3}^{13}$ got by reducing the components $\bmod \theta$ is an element of the line difference code described in section 2.4. The inner product is the usual one divided by 13. For completeness we record:

Lemma A.7. The lattice just described is isometric to the complex Leech lattice.

Proof. We will use the vectors $P_{i}$ and $L_{j}$ from the next lemma, and their inner product information from the remark after it. But we won't use that lemma itself. Write $\Lambda$ for the $\mathcal{E}$-lattice just defined. We will show that it is isometric to the complex Leech lattice, which we have denoted $\Lambda$ elsewhere in the paper. First, define $\delta_{i j}=L_{i}-L_{j}$ and $\varepsilon_{i j}=\left(P_{i}-P_{j}\right) / \psi$. The $\varepsilon_{i j}$ have the form $(0, \ldots, 0, \bar{\psi} \theta, 0, \ldots, 0,-\bar{\psi} \theta, 0, \ldots, 0)$, so they lie in $\Lambda$. We claim that $\Lambda$ is spanned by the $\delta_{i j}, \varepsilon_{i j}$ and any one $P_{i}$, say $P_{1}$. To see this, given $x \in \Lambda$, the reduction of its components modulo $\theta$ lies in the line difference code. By adding suitable multiples of $\delta_{i j}=L_{i}-L_{j}$, we may suppose without loss of generality that this codeword is the zero codeword. That is, all coordinates are divisible by $\theta$. By adding a multiple of $P_{1}$, we may suppose that the last component is 0 . It follows that all components are zero $\bmod \bar{\psi}$. Since all coordinates are divisible by $\bar{\psi} \theta$, and the coordinate sum is zero, $x$ may be expressed as a linear combination of the $\varepsilon_{i j}$.

Second, all inner products in $\Lambda$ lie in $\theta \mathcal{E}$-in particular, $\Lambda$ is integral. This is just a computation using the data in remark A.9. Third, the determinant of $\Lambda$ is 
$3^{6}$. To see this, note that the $\mathcal{E}$-span of the $\varepsilon_{i j}$ is a scaled copy of $\mathcal{E} \otimes A_{12}$, with determinant $3^{12} \cdot 13$. (Recall the factor $\frac{1}{13}$ in $\Lambda$ 's inner product.) Adjoining the $\delta_{i j}$ gives a larger lattice, whose quotient by this copy of $\mathcal{E} \otimes A_{12}$ is isomorphic to the line difference code $\mathbb{F}_{3}^{6}$, and whose determinant is $3^{6} \cdot 13$. Finally, adjoining $P_{1}$ reduces the determinant to $3^{6}$. Since $\Lambda$ has determinant $3^{6}$, and all inner products are divisible by $\theta$, it is an Eisenstein Niemeier lattice (cf. [A3, Sec. 2]). And the only Eisenstein Niemeier lattice whose isometry group contains $L_{3}(3)$ is the Leech lattice [A3, Thm. 4].

Correction to A3. This proof is similar to that of Lemma 6 in A3, which asserts that there is a unique $L_{3}(3)$-invariant integral lattice properly containing $\theta \mathcal{E} \otimes A_{12}$. This is wrong because it neglects the 13-part of the discriminant group of $\theta \mathcal{E} \otimes A_{12}$. A counterexample is $\Lambda$ in the form above. That lemma was used only once in [A3, in the proof of Lemma 9, where it is used to recognize a certain lattice as the complex Leech lattice. That result can be saved by addressing the 13-part in a manner similar to the proof above.

We will need to understand the lattice points near a particular point $C$ of $\Lambda \otimes \mathbb{C}$ that is not in $\Lambda$ itself. This will allow us to write down Leech model versions of the point-roots, line-roots and 13-points, and thereby identify the $P^{2} \mathbb{F}_{3}$ and Leech models of $L$. The name "centroid" in the next lemma is explained after lemma A.10.

Lemma A.8 (The "centroid" $C$ ). For $i=1, \ldots, 13$ define $P_{i}$ as the Leech lattice vector $\left(12 \theta, \bar{\theta}^{12}\right)$ with the $12 \theta$ in the ith position, and define $C=-P_{1} / \psi$. Then the only points of $\Lambda$ at distance $<\sqrt{42 / 13}$ from $C$ are the

$$
C+\frac{1}{\psi} P_{i}=\frac{P_{i}-P_{1}}{\psi}= \begin{cases}(0, \ldots, 0) & \text { if } i=1 \\ (-\bar{\psi} \theta, 0, \ldots, 0, \bar{\psi} \theta, 0, \ldots, 0) & \text { if } i=2, \ldots, 13\end{cases}
$$

all of which lie at distance $\sqrt{36 / 13}$.

Similarly, for $j=1, \ldots, 13$ define $L_{j}$ as the Leech lattice vector $\left((-9)^{4}, 4^{9}\right)$ with the (-9)'s in the positions corresponding to the points of $P^{2} \mathbb{F}_{3}$ that lie on the $j$ th line. Then the only points of $\frac{1}{\theta} \Lambda$ at distance $<\sqrt{14 / 13}$ from $C$ are the

$$
C-\frac{\bar{\omega}}{\theta \psi} L_{j}= \begin{cases}\frac{1}{\theta}(-9 \omega,-3 \text { (three times), }-\bar{\omega} \text { (nine times) }) & \text { if } P_{1} \in L_{j} \\ \frac{1}{\theta}(4-8 \omega,-3 \text { (four times), }-\bar{\omega} \text { (eight times) }) & \text { otherwise }\end{cases}
$$

all of which lie at distance $\sqrt{12 / 13}$.

Remark A.9. Even though the $P_{i}$ and $L_{j}$ are vectors, we write " $P_{i} \in L_{j}$ " as shorthand for "the $i$ th point and $j$ th line of $P^{2} \mathbb{F}_{3}$ are incident". The following data is useful in later calculations. The $P_{i}$ and $L_{j}$ have norm 36, and for $i \neq j$ we have $\left\langle P_{i} \mid P_{j}\right\rangle=\left\langle L_{i} \mid L_{j}\right\rangle=-3$. Also, $\left\langle P_{i} \mid L_{j}\right\rangle=-9 \theta$ or $4 \theta$ and $\left(C+\frac{1}{\psi} P_{i}\right)-\left(C-\frac{\bar{\omega}}{\theta \psi} L_{j}\right)$ has norm 3 or 4 , both according to whether or not $P_{i} \in L_{j}$. In particular, since $P_{1} \in L_{1}$ and $C+P_{1} / \psi=0$, we have $\left(C-\frac{\bar{\omega}}{\theta \psi} L_{1}\right)^{2}=3$.

Proof. To show that these vectors lie in $\Lambda$ (resp. $\frac{1}{\theta} \Lambda$ ), one just checks the conditions in Wilson's definition. Their distances from $C$ are as stated because $P_{i}^{2}=L_{j}^{2}=36$ for all $i$ and $j$. It is easy to check that all the $P_{i}$ (resp. all the $L_{j}$ ) are congruent $\bmod \psi$. The rest of the proof is like the proof of lemma A.2. That is, the lemma follows from the claim: the $P_{i}$ (resp. $L_{j}$ ) are the only norm $<42$ representatives of their $\bmod \psi$ congruence class. We treat the $P_{i}$ case; the $L_{j}$ case is the same. 
Let $v$ be a Leech vector such that $(v-C)^{2}<42 / 13$. Let $x=\psi(v-C)$. Then $x \in \Lambda$ has norm $<42$ and is distinct from all the $P_{i}$, but congruent to them $\bmod \psi$. As a nonzero member of $\psi \Lambda, x-P_{i}$ has norm at least $6 \cdot 13=78$. Since $P_{i}^{2}=36$ and $x^{2}<42$, the angle between $x$ and $P_{i}$ is obtuse. That is, in the real Euclidean space underlying $\Lambda \otimes \mathbb{C}, x$ and $P_{i}$ have negative inner product. This holds for all $i$. So $x$ has negative inner product (in this real Euclidean space) with $P_{1}+\cdots+P_{13}=0$, which is absurd.

From 2.7 recall that the Leech model for our lattice is $L \simeq \Lambda \oplus H$ where $H$ has gram matrix $\left(\begin{array}{cc}0 & \bar{\theta} \\ \theta & 0\end{array}\right)$. Wilson's definition of $\Lambda$ gives an $L_{3}(3)$ action on $L$. The $P^{2} \mathbb{F}_{3}$ model of $L$ gives another $L_{3}(3)$ action on $L$. We should note that these two $L_{3}(3)$ 's are not conjugate in $\operatorname{Aut}(L)$. The lattice pointwise fixed by the two $L_{3}(3)$ 's are $H$ and $\left(\mathcal{E} p_{\infty}+\mathcal{E} l_{\infty}\right)$ respectively and these two lattices are not isometric.

Now we can write down the point- and line-roots in the Leech model. We define $p_{i}$ to be the Leech root obtained by using $\sigma=C+\frac{1}{\psi} P_{i}$ and $\nu=1 / 2 \theta$ in (2.8). Explicitly,

$$
\begin{aligned}
p_{1} & =(0, \ldots, 0 ; 1,-\omega) \\
p_{i} & =(-\bar{\psi} \theta, 0, \ldots, 0, \bar{\psi} \theta, 0, \ldots, 0 ; 1,-\bar{\omega}) \quad \text { if } i=2, \ldots, 13
\end{aligned}
$$

where the $\bar{\psi} \theta$ appears in the $i$ th position. Similarly, we define $l_{j}$ as the root obtained from (2.6) by taking $\sigma=\left(C-\frac{\bar{\omega}}{\theta \psi} L_{j}\right) \theta \omega, m=\theta \omega, N=3$, and $\nu=\bar{\theta}$ or $\bar{\theta} / 2$ according to whether $P_{1} \in L_{j}$ or not. Explicitly, $l_{j}$ has the form

$$
l_{j}= \begin{cases}(-9 \bar{\omega},-3 \omega \text { (three times) },-1 \text { (nine times); } \theta \omega, 2 \bar{\omega}) & \text { if } P_{1} \in L_{j} \\ (4 \omega-8 \bar{\omega},-3 \omega \text { (four times) },-1 \text { (eight times); } \theta \omega, \bar{\theta}) & \text { otherwise. }\end{cases}
$$

Here the $-9 \bar{\omega}$ or $4 \omega-8 \bar{\omega}$ appears in the first position, and the $(-3 \omega)$ 's resp. (-1)'s appear in positions indexed by points of $P^{2} \mathbb{F}_{3}$ lying resp. not lying in $L_{j}$. One verifies the next lemma by direct computation.

Lemma A.10 (The point- and line-roots in the Leech model). These vectors $p_{i}$, $l_{j}$ and $\rho$ in the Leech model have the same inner products with each other as do the vectors with the same names in the $P^{2} \mathbb{F}_{3}$ model. This defines an isometry of the two models, that identifies their point-roots, line-roots and Leech cusp $\rho$. Under this identification, in the Leech model we have

$$
\begin{aligned}
p_{\infty} & =(\psi C ; \psi,-\bar{\omega} \psi)=(12 \bar{\theta}, \theta, \ldots, \theta ; \psi,-\bar{\omega} \psi) \\
l_{\infty} & =(-\bar{\omega} \theta \psi C ;-\bar{\omega} \theta \psi, 6 \bar{\omega}-\omega)=(-36 \bar{\omega}, 3 \bar{\omega}, \ldots, 3 \bar{\omega} ;-\bar{\omega} \theta \psi, 6 \bar{\omega}-\omega)
\end{aligned}
$$

Writing these vectors in the form (2.6), their values of $\sigma$ and $m$ are their entries before and immediately after the semicolon, their values of $N$ are both -3 , and their values of $\nu$ are $-\frac{13}{6} \theta$ and $-\frac{17}{2} \theta$, respectively.

Remarks. Here is how we found this identification. For each $i$ we have $\left\langle\rho \mid p_{i}\right\rangle=\theta$ in the $P^{2} \mathbb{F}_{3}$ model, so their analogues in the Leech model should have the form $\left(\sigma_{i} ; 1, \ldots\right)$ for suitable $\sigma_{i} \in \Lambda$. The inner product formula (2.7) shows that the differences between the $\sigma_{i}$ must be minimal vectors of $\Lambda$. This suggested looking for the centroid of the $\sigma_{i}$ in $\Lambda \otimes \mathbb{C}$, expecting it to have stabilizer $L_{3}(3)$ in the affine isometry group $\Lambda$ : $6 S u z$ of the Leech lattice. We were already familiar with the principle used in lemma A.2. the lattice vectors near an element of the rational span of a lattice are related to the short lattice vectors in a suitable congruence 
class. This suggested looking for a congruence class in $\Lambda$ with stabilizer $L_{3}(3)$ and exactly 13 minimal representatives. In Wilson's model of $\Lambda$ the $P_{i}$ form an $L_{3}(3)$ orbit of size 13. So we investigated and found that they are congruent mod $\psi$. This suggested that the centroid should be $C$ from lemma A.8 and that the $\sigma_{i}$ 's should be its nearest neighbors. This determined the $p_{i}$ up to their values of $\nu$. For $p_{1}$ we chose $\nu=\frac{1}{2 \theta}$ arbitrarily, and then used the orthogonality of the $p_{i}$ and (2.7) to compute the $\nu$ values of the other $p_{i}$. Since $\rho$ and the point-roots $\operatorname{span} L$ up to finite index, the expressions for all the other vectors follow.

As we mentioned after (2.7), inner products with a vector $(\sigma ; m, \ldots)$ in the Leech model can be expressed in terms of $\sigma / m \in \Lambda \otimes \mathbb{C}$. For $p_{\infty}$ and $l_{\infty}$ this point is $C$. And lemma A.8 shows that for the point-roots (resp. line-roots), the corresponding points of $\Lambda \otimes \mathbb{C}$ are $C$ 's nearest neighbors in $\Lambda$ (resp. $\frac{1}{\theta} \Lambda$ ). This will be crucial in the proofs of lemma A.11 and A.13 Finally, with additional work it is possible to introduce a version of the Leech model "centered at $C$ ", to avoid hiding some of the $L_{3}(3)$ symmetry.

Lemma A.11 (The triangle needed for step 4 in lemma 6.1, point-mirror case). Let $x$ be the projection of $\rho$ onto a point-mirror, and $T$ be the totally real triangle $\triangle \rho x p_{\infty}$. Then $T$ meets that point-mirror in $\overline{x p_{\infty}}$, meets the other point-mirrors in $p_{\infty}$ only, and misses all other mirrors.

Proof. By $L_{3}(3)$ symmetry it suffices to treat the point-root $p_{1}$. Using the Leech model, and the formula for $p_{1}$ given just before lemma A.10 we find $x=(0 ; 1 / \theta$, $-\bar{\omega} / \theta)=\left(\theta^{-1} p_{1}+\rho\right)$, which has $x^{2}=\langle\rho \mid x\rangle=-1$. The other two vertices of $T$ are represented by $p_{\infty}$ from lemma $\mathrm{A} .10$ and $\rho=(0 ; 0,1)$. In calculations we will use $\bar{\theta} \bar{\psi} p_{\infty}$ rather than $p_{\infty}$, because it has negative inner product (namely -39 ) with both $\rho$ and $x$. So we will be able to apply lemma A.1.

First we claim that $T$ lies in the union of the second critical horoball around $\rho$ and the second critical ball around $p_{\infty}$. To see this, note that $\operatorname{ht}_{\rho}(x)=1$, so $x$ lies on the boundary of the first critical horoball. (In fact this holds by construction.) Also, one checks ht ${ }_{\rho}\left(p_{\infty}\right)=13>3$, so $p_{\infty}$ lies outside the second critical horoball. Therefore it suffices to find where the boundary of this horoball intersects $\overline{p_{\infty} \rho}$ and $\overline{p_{\infty} x}$, and check that both points lie in the second critical ball around $p_{\infty}$. Since $\overline{p_{\infty} \rho}$ travels directly toward the horoball, its intersection point is closer to $p_{\infty}$ than is the intersection point of $\overline{p_{\infty} x}$. So it suffices to show that this second intersection point lies in the second critical horoball. We found this intersection point by the method from the proof of lemma A.4 obtaining $\bar{\theta} \bar{\psi} p_{\infty}+(9 \sqrt{26}-39) x$. This can be verified by checking that it has height 3 . It has norm -702 and its inner product with $\bar{\theta} \bar{\psi} p_{\infty}$ is $1404-351 \sqrt{26}$. So the point of $\mathbb{B}^{13}$ it represents lies at distance $\cosh ^{-1} \sqrt{63-12 \sqrt{26}} \approx .810$ from $p_{\infty}$. This is less than $r_{2}=\sinh ^{-1}(1) \approx .881$, so this point lies in the second critical ball, as desired.

Therefore the only mirrors that might meet $T$ are the Leech mirrors (which include the batch 0 roots, namely the point-roots) and the batch 1 mirrors around $p_{\infty}$. We used computer calculations in the $P^{2} \mathbb{F}_{3}$ model, and lemma A.1 to check that the batch 1 mirrors miss $T$. So it remains to examine how the mirror of a Leech root $s$ can meet $T$. This part of the proof is similar to the proof of lemma A.3. we will compute $\langle T \mid s\rangle \subseteq \mathbb{C}$ and examine whether it contains the origin. For use in the calculation we tabulate the parameters of the important vectors when they are 
written as in (2.6):

$\begin{array}{cccccc} & & \sigma & m & N & \nu \\ x & (\text { a vertex of } T) & 0 & 1 / \theta & -1 & -\theta / 18 \\ \bar{\theta} \bar{\psi} p_{\infty} & (\text { a vertex of } T) & -13 \theta C & -13 \theta & -117 & -169 \theta / 2 \\ s & \text { (a Leech root) } & \sigma & 1 & 3 & \nu\end{array}$

The inner product formula (2.7) and some simplification gives

$$
\begin{aligned}
\langle x \mid s\rangle & =\frac{\theta}{6} \sigma^{2}-\frac{\theta}{3}(3 \nu+\theta / 2) \\
\left\langle\bar{\theta} \bar{\psi} p_{\infty} \mid s\right\rangle & =\frac{13 \theta}{2}\left((C-\sigma)^{2}-\frac{36}{13}\right)-13 \theta(\operatorname{Im}\langle C \mid \sigma\rangle+3(\nu+\theta / 6))
\end{aligned}
$$

The first terms are their imaginary parts. So $\langle x \mid s\rangle$ lies on the real axis (if $\sigma=0$ ) or above it (otherwise). Also, lemma A.8 gives exactly the inequality needed to make $\left\langle\bar{\theta} \bar{\psi} p_{\infty} \mid s\right\rangle$ lie on or above the real axis, namely that $C-\sigma$ has norm $\geq \frac{36}{13}$. It is something of a miracle that the geometry of the Leech lattice gives us exactly the bound that we need for our argument to work. Finally, $\langle\rho \mid s\rangle=\theta$ since $s$ is a Leech root. So $\langle T \mid s\rangle$ lies in the closed upper half plane. If $\sigma \neq 0$ then two of its vertices lie strictly above the real axis, so $\langle T \mid s\rangle$ misses the origin unless the third vertex coincides with the origin, i.e., unless $\left\langle\bar{\theta} \bar{\psi} p_{\infty} \mid s\right\rangle=0$. But then $s$ is a point-root, since these are the only roots orthogonal to $p_{\infty}$. So suppose $\sigma=0$. Then the above formulas simplify to the real numbers $\langle x \mid s\rangle=-\theta(\nu+\theta / 6)$ and $\left\langle\bar{\theta} \bar{\psi} p_{\infty} \mid s\right\rangle=-39 \theta(\nu+\theta / 6)$. (The latter uses $C^{2}=36 / 13$.) Since these differ by a positive factor, the only way $\langle T \mid s\rangle$ can contain the origin is for both of them to vanish. In particular $\left\langle p_{\infty} \mid s\right\rangle=0$, so again $s$ is a point-root, indeed $p_{1}$.

The analogue of lemma A.11 for a line-mirror requires a much larger tabulation of the mirrors near $p_{\infty}$ than we have needed so far. The following lemma describes batches $0, \ldots, 5$ in a manner suitable for machine computation. Batches 0, 1 and 2 appear in table 1 In batches 3, 4 and 5 there are 743418,107953560 and 480961338 mirrors. To actually construct the batches one should refer to the tabulation of the line code in tables 2 and 3 of $\mathrm{A3}$.

Lemma A.12 (Mirrors near a 13-point). Write vectors of $L$ in the $P^{2} \mathbb{F}_{3}$ model. Then the mirrors in batches $b=0, \ldots, 5$, i.e., those at distance $<\sinh ^{-1} 2 \approx 1.444$ from $p_{\infty}$, are the orthogonal complements of the roots $s=\left(s_{0} ; s_{1}, \ldots, s_{13}\right)$ described in the next paragraph. For $b=0$ this gives all roots, while for the other cases it gives one from each scalar class.

Define the "desired norm" $N=3,4,6,7,10$ or 12 and the "required coordinate sum" $S=0,1,0,1,1$, or $0 \in \mathbb{F}_{3}$ according to the value of $b=0, \ldots, 5$. Choose any codeword $w=\left(w_{1}, \ldots, w_{13}\right)$ in the line code whose weight is at most $N$ and whose coordinate sum is $S$. Choose $e_{1}, \ldots, e_{13}$ with $e_{i} \in\{0,3,6\}$ if $w_{i} \neq 0$ and $e_{i} \in\{0,3,9,12\}$ if $w_{i}=0$, such that the sum of the $e_{i}$ equals $N-\operatorname{weight}(w)$. Choose any Eisenstein integers $s_{1}, \ldots, s_{13}$ such that $s_{i} \bmod \theta$ is $w_{i}$, and $\left|s_{i}\right|^{2}$ is either $e_{i}$ or $e_{i}+1$ according to whether $w_{i}=0$ or $w_{i} \neq 0$. Prefix the coordinates $\left(s_{1}, \ldots, s_{13}\right)$ by a coordinate $s_{0}=0,1, \theta,-2,2-(\omega$ or $\bar{\omega})$, or 3 according to value of $b=0, \ldots, 5$.

Proof. Suppose $s=\left(s_{0} ; s_{1}, \ldots, s_{13}\right)$ is a root in one of the batches $0, \ldots, 5$. Then $\left|\left\langle s \mid p_{\infty}\right\rangle\right|^{2}<3 \cdot 12$, which boils down to the condition $\left|s_{0}\right|^{2}<12$, i.e., $\left|s_{0}\right|^{2} \in$ $\{0,1,3,4,7,9\}$. If $\left|s_{0}\right|^{2}=0$ then of course $s_{0}=0$. In the other cases there is a 
unique way to scale $s$ by a unit, such that $s_{0}$ is as described at the end of the lemma. In all cases, $s^{2}=3$ says that the vector $\left(s_{1}, \ldots, s_{13}\right)$ has norm $N$, and the definition of $L$ requires that its reduction $w$ modulo $\theta$ is in the line code and has coordinate sum $S$. For $i=1, \ldots, 13$ we take $e_{i}$ to be defined as $\left|s_{i}\right|^{2}$ if $w_{i}=0$, or $\left|s_{i}\right|^{2}-1$ if $w_{i} \neq 0$. If $w_{i}=0$ then $s_{i}$ must be divisible by $\theta$, and $\left|s_{i}\right|^{2} \leq 12$, so $e_{i} \in\{0,3,9,12\}$. If $w_{i} \neq 0$ then $N \leq 12$ gives $\left|s_{i}\right|^{2} \leq 9$ since $w_{i}$ has weight at least 4 (so there are at least 3 other nonzero coordinates). An element of $\mathcal{E}$ not divisible by $\theta$, and having norm $\leq 9$, must have norm 1,4 or 7 . That is, when $w_{i} \neq 0$ we have proven $e_{i} \in\{0,3,6\}$. We have just established the lemma's constraints on the $e_{i}$. The constraints on the $s_{i}$ in terms of the $e_{i}$ are satisfied by the construction of the $e_{i}$. Conversely, if one follows the instructions in choosing $w, e_{1}, \ldots, e_{13}, s_{1}, \ldots, s_{13}, s_{0}$ then one obtains a norm 3 vector of $L$ in the specified batch.

Lemma A.13 (The triangle needed for step 4 in lemma 6.1 line-mirror case). Let $x$ be the projection of $\rho$ onto a line-mirror, and $T$ be the totally real triangle $\triangle \rho x l_{\infty}$. Then $T$ meets that line-mirror in $\overline{x l_{\infty}}$, meets the other line-mirrors in $l_{\infty}$ only, and misses all other mirrors.

Proof. This is similar to lemma A.11 but there are some new issues. By symmetry we may take the line root to be $l_{1}$. Computation gives $x=\rho-\bar{\omega} l_{1}$, with $x^{2}=$ $\langle\rho \mid x\rangle=-3$. In computations we use $\omega \bar{\psi} l_{\infty}$ in place of $l_{\infty}$ because it has negative inner product with $\rho$ and $x$, namely -39 . This is also its norm.

We claim that $T$ lies in the union of the sixth critical ball around $l_{\infty}$ and the third critical horoball around $\rho$. Following the proof of lemma A.11, it is enough to check that the point where $\overline{l_{\infty} x}$ pierces the boundary of the 3rd critical horoball lies in the 6 th critical ball. One can check that this point is represented by the vector $\omega \bar{\psi} l_{\infty}+(4 \sqrt{39}-13) x$, by computing that its height is 4 . Its norm is -1404 and its inner product with $\omega \bar{\psi} l_{\infty}$ is $468-156 \sqrt{39}$. This lets us compute the distance from this point to $l_{\infty}$, namely $\cosh ^{-1} \sqrt{\frac{64}{3}-\frac{8}{3} \sqrt{39}} \approx 1.407$. This is less than $r_{6}=\sinh ^{-1}(2) \approx 1.444$, as desired.

So the only mirrors that could meet $T$ are the first and second shell mirrors around $\rho$ (this includes the line mirrors, which are the batch 0 roots around $l_{\infty}$ ), and the mirrors in batches $1, \ldots, 5$ around $l_{\infty}$. The latter checks are done in the $P^{2} \mathbb{F}_{3}$ model. We applied an isometry $F$ of $L$ that exchanges the point- and lineroots up to signs, namely $p_{i} \mapsto l_{14-i}, l_{j} \mapsto-p_{14-j}$. (It follows that $F\left(p_{\infty}\right)=$ $l_{\infty}$ and $F\left(l_{\infty}\right)=-p_{\infty}$, which is useful for writing down a matrix for $F$.) So it suffices to check that the triangle with vertices $F(\rho), F(x)$ and $F\left(\omega \bar{\psi} l_{\infty}\right)$ misses all batch $1, \ldots, 5$ mirrors around $p_{\infty}$. For this we enumerated these mirrors in the $P^{2} \mathbb{F}_{3}$ model by using lemma $\mathrm{A} .12$, and checked that they all miss this triangle by using lemma A.1. This was the only computer calculation in the paper that took more than a moment - sixteen hours on a laptop.

To examine how the Leech mirrors and second-shell mirrors meet $T$, we return to the Leech model. We begin by writing down the parameters $\sigma, m, N$ and $\nu$ 
when we write the following important vectors in the form (2.6):

$\begin{array}{cccccc} & & \sigma & m & N & \nu \\ x & \text { (a vertex of } T) & -\left(C-\frac{\omega}{\theta \psi} L_{1}\right) \theta & -\theta & -3 & -\theta \\ \omega \bar{\psi} l_{\infty} & \text { (a vertex of } T) & -13 \theta C & -13 \theta & -39 & -\frac{221}{2} \theta \\ s & \text { (a Leech root) } & \sigma & 1 & 3 & \nu \\ \text { or } s & \text { (a second-shell root) } & \sigma & \theta & 3 & \nu\end{array}$

Again we will use lemma A.1 to determine whether $s^{\perp}$ meets $T$. We begin with the case of a second-shell root because it is simpler. The real part of the inner product formula (2.7) is

$$
\begin{aligned}
\operatorname{Re}\langle x \mid s\rangle & =\frac{3}{2}\left(\frac{\sigma}{\theta}-\left(C-\frac{\bar{\omega}}{\theta \psi} L_{1}\right)\right)^{2} \\
\operatorname{Re}\left\langle\omega \bar{\psi} l_{\infty} \mid s\right\rangle & =\frac{39}{2}\left(\left(\frac{\sigma}{\theta}-C\right)^{2}-\frac{12}{13}\right)
\end{aligned}
$$

We see that (A.3) is at least 0 , with equality if and only if $\frac{\sigma}{\theta}=C-\frac{\bar{\omega}}{\theta \psi} L_{1}$. And (A.4) is at least 0 by lemma A.8. Together with $\operatorname{Re}\langle\rho \mid s\rangle=3$, we see that $\langle T \mid s\rangle$ lies in the closed right half plane. If $\frac{\sigma}{\theta} \neq C-\frac{\bar{\omega}}{\theta \psi} L_{1}$ then (A.3) is strictly positive, so the only way $\langle T \mid s\rangle$ could contain the origin is for its vertex $\left\langle\omega \bar{\psi} l_{\infty} \mid s\right\rangle$ to be the origin. That is, $s \perp l_{\infty}$, so $s$ is a line root.

So now suppose $\frac{\sigma}{\theta}=C-\frac{\bar{\omega}}{\theta \psi} L_{1}$. This implies that $\left(s-\bar{\omega} l_{1}\right)$ is a multiple of $\rho$ (just write $s$ and $l_{1}$ in the Leech coordinate system). Since $s$ and $l_{1}$ both have norm 3 , it quickly follows that $s=\bar{\omega} l_{1}+n \theta \rho$ for some integer n. Using the known inner products between points, lines, $l_{\infty}$ and $\rho$, we find that

$$
\left\langle\omega \bar{\psi} l_{\infty} \mid s\right\rangle=39 n \theta \text { and }\langle x \mid s\rangle=3 n \theta .
$$

Since these differ by a positive factor, the only way 0 could lie in $\langle T \mid s\rangle$ is for both of them to be 0 . Then $s$ is a line root, indeed $l_{1}$.

Now suppose $s$ is a Leech root. The imaginary part of (2.7) is

$$
\begin{aligned}
\operatorname{Im}\langle x \mid s\rangle & =\frac{\theta}{2}\left[\left(\sigma-\left(C-\frac{\bar{\omega}}{\theta \psi} L_{1}\right)\right)^{2}-2\right] \\
\operatorname{Im}\left\langle\omega \bar{\psi} l_{\infty} \mid s\right\rangle & =\frac{13 \theta}{2}\left((C-\sigma)^{2}-\frac{38}{13}\right)
\end{aligned}
$$

We have $\langle\rho \mid s\rangle=\theta$, which lies above the real axis. We claim that A.5 does also. To see this, recall from remark A.9 that $C-\frac{\bar{\omega}}{\theta \psi} L_{1} \in \frac{1}{\theta} \Lambda$ has norm 3. So it equals a norm 9 vector of $\Lambda$, divided by $\theta$. By lemma A.2, the distance from $C-\frac{\bar{\omega}}{\theta \psi} L_{1}$ to $\Lambda$ is $\sqrt{3}$. So the first term in the brackets in A.5 is at least 3. So A.5 lies above the real axis. Next, lemma A.8 says that either $(C-\sigma)^{2} \geq \frac{42}{13}$ or else $\sigma=C+\frac{1}{\psi} P_{i}$ for some $i=1, \ldots, 13$. In the first case we see that (A.6) lies above the real axis, so $T$ does too, so it cannot contain the origin.

So suppose $\sigma=C+\frac{1}{\psi} P_{i}$. This implies that $\left(s-p_{i}\right)$ is a multiple of $\rho$. Since $s$ and $p_{1}$ both have norm 3 , it follows that $s=p_{i}+n \rho$ for some integer $n$. Using the known inner products between points, lines, $l_{\infty}$ and $\rho$, we find that

$$
\begin{aligned}
\langle x \mid s\rangle & = \begin{cases}\theta-3 n+\bar{\omega} \theta & \text { if } P_{i} \in L_{1} \\
\theta-3 n & \text { otherwise. }\end{cases} \\
\left\langle\omega \bar{\psi} l_{\infty} \mid s\right\rangle & =\bar{\theta}+6-39 n
\end{aligned}
$$


(Note that (A.7) is above the real axis and (A.8) is below it.) We want to determine whether the origin lies in the triangle $\langle T \mid s\rangle$ that has (A.8), (A.7) and $\langle\rho \mid s\rangle=\theta$ for vertices. We can find the intersection of $\langle T \mid s\rangle$ with $\mathbb{R}$ by writing $A$, resp. $B$, for the convex combination of $\left\langle\omega \bar{\psi} l_{\infty} \mid s\right\rangle$ and $\langle\rho \mid s\rangle$, resp. $\langle x \mid s\rangle$, that has no imaginary part. Then the origin lies in $\langle T \mid s\rangle$ just if it lies in the interval with endpoints $A$ and $B$. One works out $A$ and $B$, with the result

$$
A=3-\frac{39}{2} n \quad B= \begin{cases}3-15 n & \text { if } P_{i} \in L_{1} \\ 3-21 n & \text { otherwise. }\end{cases}
$$

If $n \leq 0$ then $A$ and $B$ are both positive. And if $n \geq 1$ then $A$ and $B$ are both negative. So the origin does not lie in $\langle T \mid s\rangle$.

\section{Appendix B. How two COMPlex triangles MeEt the MIRRors}

In this appendix we prove lemma $\overline{B .2}$ the complex triangles $\triangle \rho \tau p_{\infty}$ and $\triangle \rho \tau l_{\infty}$ miss the mirror arrangement except at $p_{\infty}$ and $l_{\infty}$ respectively. This is the key fact in step 2 in the proof of lemma 6.1] Because these triangles are complex instead of totally real, we cannot use the machinery in appendix A. Instead we exploit the fact that they lie in $\mathbb{B}(F) \cong \mathbb{B}^{1}$, where $F$ is the $L_{3}(3)$-invariant sublattice of $L$. Our first step is to understand how this $\mathbb{B}^{1}$ meets the mirrors:

Lemma B.1. Suppose $x \in \mathbb{B}^{1} \cap \mathcal{H}$. Then either $x$ is represented by a norm -3 vector of $F$, or is orthogonal to a norm 3 vector of $F$.

Proof. Write $M$ for the sublattice of $L$ spanned by the roots orthogonal to $x$. The lattice $M$ is non-empty because $x \in \mathcal{H}$. So $1 \leq \operatorname{dim} M \leq 13$. As an $\mathcal{E}$-lattice with all inner products divisible by $\theta$, and spanned by roots, $M$ is a direct sum of copies of the Eisenstein root lattices $A_{2}^{\mathcal{E}}, D_{4}^{\mathcal{E}}, E_{6}^{\mathcal{E}}$ and $E_{8}^{\mathcal{E}}$. (See [A3, Thm. 3].) There are at most 13 direct summands of $M$ and $L_{3}(3)$ acts on the set of these. Since $L_{3}(3)$ is simple and contains an element of order 13, the smallest nontrivial permutation representations of $L_{3}(3)$ are on 13 objects. Therefore either (a) $L_{3}(3)$ preserves each direct summand of $M$ or (b) $M$ is the sum of 13 copies of $A_{2}^{\mathcal{E}}$.

First we treat case (a). The action of $L_{3}(3)$ on $F^{\perp}$ is known and irreducible: it is the deleted permutation representation coming from $L_{3}(3)$ 's action on the points of $P^{2} \mathbb{F}_{3}$. So as an $L_{3}(3)$ representation, $x^{\perp}$ decomposes into two irreducible factors: $F^{\perp}$ and a one dimensional representation. We are assuming that the underlying vector space of each direct summand of $M$ is preserved by $L_{3}(3)$ and so must contain one of these irreducible factors. Since each summand of $M$ have dimension at most 4 , it follows that $M$ must isomorphic to $A_{2}^{\mathcal{E}}$ and lie in the fixed space of $L_{3}(3)$. So $F$ contains a root orthogonal to $x$.

Now we treat case (b). As the orthogonal complement of 13 mutually orthogonal roots, the line in $L \otimes \mathbb{C}$ corresponding to $x$ is represented by a lattice vector; we choose a primitive one and use the same name $x$ for it. The product of the $\bar{\omega}-$ reflections in the 13 roots, times the scalar $\omega$, acts on $L$ by scaling $x$ by $\omega$ and fixing $x^{\perp}$ pointwise. So it is given by the same formula (2.4) as a reflection, namely $v \mapsto v-(1-\omega)\langle v \mid x\rangle x / x^{2}$. (This differs from a reflection in that its fixed set in $\mathbb{B}^{13}$ is a point not a hyperplane.) Since this preserves $L$, we have $(1-\omega)\langle v \mid x\rangle / x^{2} \in \mathcal{E}$ for every $v \in L$. Since $L=\theta \cdot L^{*}$, there exists $v \in L$ with $\langle v \mid x\rangle=1-\bar{\omega}$. So $3 / x^{2} \in \mathcal{E}$, which forces $x^{2}=-3$. 
It is easy to see that $F$ is spanned by $\rho=(-\bar{\psi} ;-1, \ldots,-1)$ and $p_{\infty}=(\bar{\theta} ; 0, \ldots, 0)$. These have norms 0 and -3 , and $\left\langle\rho \mid p_{\infty}\right\rangle=\theta \bar{\psi}$. We will also use a second null vector $\rho^{\prime}$, which is defined as $-\omega$ times the image of $\rho$ under the $\omega$ - "reflection" in $p_{\infty}$. (As in the proof above, this fixes a single point of $\mathbb{B}^{13}$ rather than a hyperplane.) One can check $\rho^{\prime}=\bar{\psi} p_{\infty}-\omega \rho=(\bar{\omega} \bar{\psi} ; \omega, \ldots, \omega)$ and $\left\langle\rho \mid \rho^{\prime}\right\rangle=13 \theta$.

Everything becomes easier if we work in a certain superlattice $E$ of $F$, namely the one spanned by $\rho / \bar{\psi}$ and $\rho^{\prime} / \bar{\psi}$. We use the notation $[u, v]$ to mean $\left(u \rho^{\prime}+v \rho\right) / \bar{\psi}$. Obviously $E$ contains $\rho$, and it contains $p_{\infty}$ by our formula for $\rho^{\prime}$ in terms of $p_{\infty}$ and $\rho$. So $E$ does indeed contain $F$. The main advantage of working in $E$ is that the inner product has the simple form

$$
\left\langle[u, v] \mid\left[u^{\prime}, v^{\prime}\right]\right\rangle=\left(\begin{array}{ll}
u & v
\end{array}\right)\left(\begin{array}{ll}
0 & \bar{\theta} \\
\theta & 0
\end{array}\right)\left(\begin{array}{l}
\bar{u}^{\prime} \\
\bar{v}^{\prime}
\end{array}\right)
$$

which is the same as in (2.5). One can check that $p_{\infty}=[1, \omega]$ and $l_{\infty}=[\bar{\theta} \bar{\omega}, \omega-2]$. Now we can prove the main result of this appendix:

Lemma B.2 (Two triangles needed in step 2 of lemma 6.1). The only point of $\mathcal{H}$ in $\triangle \rho \tau p_{\infty}\left(\right.$ resp. $\left.\triangle \rho \tau l_{\infty}\right)$ is $p_{\infty}\left(\right.$ resp. $\left.l_{\infty}\right)$.

Proof. Because $\tau$ is the midpoint of $\overline{p_{\infty} l_{\infty}}$, the union of these two triangles is the larger triangle $T=\triangle \rho p_{\infty} l_{\infty}$. So it suffices to show that this triangle misses $\mathcal{H}$ except at $p_{\infty}$ and $l_{\infty}$. We identify the projective space of $F \otimes \mathbb{C}$ with $\mathbb{C} \cup\{\infty\}$ by plotting a vector $[u, v]$ as $v / u$. Then $\mathbb{B}^{1}$ corresponds to the upper half plane, and $\rho, p_{\infty}$ and $l_{\infty}$ correspond to $\infty, \omega$ and $\frac{3}{2}+\frac{\theta}{6}$ respectively. So $T$ is the hyperbolic triangle they span. The edges of $T$ are the vertical lines through $\omega,\left(\frac{3}{2}+\frac{\theta}{6}\right)$ and an arc $C$ of the circle $\left\{z:\left|z-\frac{1}{3}\right|^{2}<\frac{13}{9}\right\}$. The image of $\tau$ in the upper half plane is $(1+i)$, which is the midpoint of the circular arc $C$.

Our strategy is to find all the norm -3 vectors of $F$ representing points of $T$, and all norm 3 vectors orthogonal to points of $T$, and then apply lemma B.1. It is convenient to find all such vectors in $E$ first, and then discard the ones that lie outside $F$. We begin by writing an arbitrary norm -3 vector of $E$ as $x=$ $\left[m, \frac{\theta}{\bar{m}}\left(\frac{0-(-3)}{6}+\nu\right)\right]$, where $m \in \mathcal{E}-\{0\}$, and $\nu \in \operatorname{Im} \mathbb{C}$ is chosen so that the second component lies in $\mathcal{E}$. (This is just like the analysis leading to (2.6).) The corresponding point of $\mathbb{B}^{1}$ has imaginary part $\theta / 2|m|^{2}$. Now suppose $x \in T$. The only point of $T$ with imaginary part $\theta / 6$ is $l_{\infty}$, and all its other points have larger imaginary part. Therefore either $x$ is a multiple of $l_{\infty}$, or else $|m|=1$. In the latter case we scale $x$ so that $m=1$, so $x=\left[1, \theta\left(\frac{1}{2}+\nu\right)\right]$. Since the second component lies in $\mathcal{E}$, we have $\nu=\frac{1}{2 \theta}+\frac{n}{\theta}$ for some $n \in \mathbb{Z}$. Then the point of $\mathbb{B}^{1}$ represented by $x$ is $n-\bar{\omega}$. Since every point of $T$ has real part at least $-\frac{1}{2}$ and at most $\frac{3}{2}$, the only possibilities for $n$ are $-1,0$ and 1 . The case $n=-1$ yields $x=p_{\infty}$. The case $n=0$ does not actually arise, because $-\bar{\omega}$ lies below the geodesic $\overline{p_{\infty} l_{\infty}}$. The case $n=1$ yields a point of $T$. We have shown that the only points of $T$ represented by norm -3 vectors of $E$ are $p_{\infty}, l_{\infty}$ and $[1,1-\bar{\omega}]$.

Now consider a point of $T$ orthogonal to a norm 3 vector of $E$, say $[u, v]$. The orthogonal complement of $[u, v]$ is spanned by $[\bar{u}, \bar{v}]$, which is a norm -3 vector of $E$. So we have shown that the only points of $T$ that are represented by a norm -3 vectors of $E$, or are orthogonal to a norm 3 vector of $E$, are $p_{\infty}, l_{\infty}$ and $[1,1-\bar{\omega}]$.

Finally, consider a point of $T \cap \mathcal{H}$ other than $p_{\infty}$ and $l_{\infty}$. By lemma B.1, either it is represented by a norm -3 vector of $F$, or is orthogonal to a norm 3 vector of 
$F$. This norm \pm 3 vector lies in $E$, so the previous two paragraphs show that the point is $[1,1-\bar{\omega}]$. So it suffices to show that $[1,1-\bar{\omega}] \notin \mathcal{H}$. For this we observe that $F$ contains neither the norm -3 vector $[1,1-\bar{\omega}]$, nor the norm 3 vector $[1,1-\omega]$ orthogonal to it.

\section{REFERENCES}

[A1] D. Allcock, The Leech lattice and complex hyperbolic reflections, Invent. Math. 140 (2000) 283-301.

[A2] D. Allcock, A monstrous proposal, in Groups and Symmetries, From neolithic Scots to John McKay, ed. J. Harnad. AMS and CRM, (2009). arXiv:math/0606043

[A3] D. Allcock, On the Y555 complex reflection group, J. Alg. 322, no. 5 (2009) 1454-1465.

$[\mathrm{AB}]$ D. Allcock and T. Basak, Geometric generators for braid like groups, Geom. Topol. 20 (2016), no. 2, 747-778. arXiv:1403:2401

[ACT1] D. Allcock, J. Carlson and D. Toledo, The complex hyperbolic geometry of the moduli space of cubic surfaces, J. Algebraic Geom. 11 (2002) 659-724.

[ACT2] D. Allcock, J. Carlson and D. Toledo, The moduli space of cubic threefolds as a ball quotient, Mem. Amer. Math. Soc. 209 (2011). ISBN 978-0-8218-4751-0.

[Ban] E. Bannai, Fundamental groups of the spaces of regular orbits of the finite unitary reflection groups of dimension 2, J. Math. Soc. Japan 28 (1976), Number 3, 447-454.

[Ba1] T. Basak, The complex Lorentzian Leech lattice and the bimonster, J. Alg. 309 (2007) $32-56$.

[Ba2] T. Basak, On Coxeter diagrams of complex reflection groups, Trans. Amer. Math. Soc. 364 (2012), no. 9, 4909-4936.

[Ba3] T. Basak, The complex Lorentzian Leech lattice and the bimonster II, Trans. Amer. Math. Soc. 368 (2016), no. 6, 4171-4195. arXiv:0811.0062

[Be] D. Bessis, Finite complex reflection arrangements are $K(\pi, 1)$. Ann. of Math. (2) 181 (2015), no. 3, 809-904.

[BH] M. Bridson and A. Haefliger, Metric spaces of non-positive curvature, Grundlehren der Mathematischen Wissenschaften, 319. Springer-Verlag, Berlin, 1999.

[Br1] E. Brieskorn, Die Fundamentalgruppe des Raumes der regulären Orbits einer endlichen komplexen Spiegelungsgruppe, Invent. Math. 12 (1971) 57-61.

[Br2] E. Brieskorn, Vue densemble sur les problèmes de monodromie, in: Singularités `a Cargèse, Rencontre sur les Singularités en Géométrie Analytique, Inst. Études sci. de Cargèse, 1972, Asterisque, Nos. 7 et 8, Soc. Math. France, Paris, 1973, pp. 393413.

[ATLAS] J. H. Conway et. al., Atlas of finite groups. Oxford University Press, Eynsham, 1985.

[CSi] J. H. Conway and C. S. Simons, 26 Implies the Bimonster, J. Alg. 235, (2001) 805-814.

[CSl] J. H. Conway, and N. J. A. Sloane, Sphere Packings, Lattices and Groups 3rd Ed. SpringerVerlag (1998)

[FN] R. Fox and L. Neuwirth, The braid groups, Math. Scand. 10 (1962) 119-126.

[Go] W. Goldman, Complex hyperbolic geometry Oxford mathematical monographs (1999).

[La] R. Laza, Deformations of singularities and variation of GIT quotients. Trans. Amer. Math. Soc. 361 (2009), no. 4, 2109-2161.

[Li] Libgober A. Libgober, On the fundamental group of the space of cubic surfaces. Math. Z. 162 (1978), no. 1, 63-67.

[Lö] Lönne, Michael Fundamental group of discriminant complements of Brieskorn-Pham polynomials. C. R. Math. Acad. Sci. Paris 345 (2007), no. 2, 93-96.

[L1] E. Looijenga, The smoothing components of a triangle singularity. II. Math. Ann. 269 (1984), no. $3,357-387$.

[L2] E. Looijenga, Compactifications defined by arrangements. II. Locally symmetric varieties of type IV. Duke Math. J. 119 (2003), no. 3, 527-588.

[L3] E. Looijenga, Artin groups and the fundamental groups of some moduli spaces. J. Topol. 1 (2008) 187-216.

[Mi] M. Broué, Introduction to complex reflection groups and their braid groups. Lecture Notes in Mathematics, 1988. Springer-Verlag, Berlin, 2010.

[vdL] H. van der Lek, The homotopy type of complex hyperplane complements, thesis, Katholieke Universiteit te Nijmegen, 1983. 
[W] R. Wilson, The complex Leech Lattice and maximal subgroups of the Suzuki group. J. Algebra 84 (1983), 151-188.

Department of Mathematics, University of Texas, Austin

E-mail address: allcock@math.utexas.edu

$U R L:$ http://www. math. utexas.edu/ ${ }^{\sim}$ allcock

Department of Mathematics, Iowa State University, Ames IA, 50011.

E-mail address: tathastu@gmail.com

$U R L:$ https://orion.math.iastate.edu/tathagat/ 